Florida International University FIU Digital Commons

6-27-2012

\title{
An Experimental and Theoretical Analysis of Nitric Oxide in the Microcirculation
}

Shabnam M. Namin

Florida International University, snami001@fiu.edu

DOI: $10.25148 /$ etd.FI12080622

Follow this and additional works at: https://digitalcommons.fiu.edu/etd

\section{Recommended Citation}

Namin, Shabnam M., "An Experimental and Theoretical Analysis of Nitric Oxide in the Microcirculation" (2012). FIU Electronic Theses and Dissertations. 699.

https://digitalcommons.fiu.edu/etd/699 


\section{FLORIDA INTERNATIONAL UNIVERSITY}

Miami, Florida

AN EXPERIMENTAL AND THEORETICAL ANALYSIS OF NITRIC OXIDE AVAILABILITY IN THE MICROCIRCULATION

A dissertation submitted in partial fulfillment of the requirements for the degree of DOCTOR OF PHILOSOPHY in BIOMEDICAL ENGINEERING by

Shabnam M Namin 
To: Dean Amir Mirmiran

College of Engineering and Computing

This dissertation, written by Shabnam M Namin, and entitled An Experimental and Theoretical Analysis of Nitric Oxide in the Microcirculation, having been approved in respect to style and intellectual content is referred to you for judgment.

We have read this dissertation and recommend that it be approved.

Wei-Chiang Lin

Konstantinos Kavallieratos

Yen-Chih Huang

Anthony McGoron

Nikolaos Tsoukias, Major Professor

Date of Defense: June 27, 2012

The dissertation of Shabnam M Namin is approved.

Dean Amir Mirmiran
College of Engineering and Computing

Florida International University, 2012 


\section{DEDICATION}

I dedicate this dissertation to my family, especially my father. Without their patience, understanding, support, and most of all love, the completion of this work would not have been possible. After all the blood, sweat and tears, I know we all could not wait any longer for this day to come! 


\section{ACKNOWLEDGMENTS}

I wish to thank the members of my lab for assisting me especially with my mathematical modeling. Dr. Sridevi Nagaraja, Dr. Adam Kapella, and soon to be Dr. Jaimit Parikh were extremely helpful with this. Coding was always a difficult aspect for me, and I appreciate their patience and help. I would also like to thank my post-doc, Dr. Mahesh Joshi. He was always there to answer any questions and help me overcome any experimental obstacles. Soon to be Drs. Tushar Gadkari and Kumpal Madrasi were my fellow experimentalist and also assisted me any time I needed. In addition, I want to thank my major professor, Dr. Nikolaos Tsoukias for assisting me throughout my studies and research. It was a privilege to work for such a brilliant person and to have this person as my mentor and friend. Finally, I want to acknowledge the University Graduate

School for awarding me the Dissertation Year Fellowship (DYF). It allowed me to completely focus on completing my dissertation and finishing in a timely manner. 
ABSTRACT OF THE DISSERTATION

AN EXPERIMENTAL AND THEORETICAL ANALYSIS OF NITRIC OXIDE

AVAILABILITY IN THE MICROCIRCULATION

by

Shabnam M Namin

Florida International University, 2012

Miami, Florida

\section{Professor Nikolaos Tsoukias, Major Professor}

Nitric Oxide (NO) is produced in the vascular endothelium where it then diffuses to the adjacent smooth muscle cells (SMC) activating agents known to regulate vascular tone. The close proximity of the site of NO production to the red blood cells (RBC) and its known fast consumption by hemoglobin, suggests that the blood will scavenge most of the NO produced. Therefore, it is unclear how NO is able to play its role in accomplishing vasodilation. Investigation of NO production and consumption rates will allow insight into this paradox.

DAF-FM is a sensitive NO fluorescence probe widely used for qualitative assessment of cellular NO production. With the aid of a mathematical model of NO/DAF-FM reaction kinetics, experimental studies were conducted to calibrate the fluorescence signal showing that the slope of fluorescent intensity is proportional to $[\mathrm{NO}]^{2}$ and exhibits a saturation dependence on [DAF-FM]. In addition, experimental data exhibited a $\mathrm{K}_{\mathrm{m}}$ dependence on [NO]. This finding was incorporated into the model elucidating $\mathrm{NO}_{2}$ as the possible activating agent of DAF-FM. A calibration procedure 
was formed and applied to agonist stimulated cells, providing an estimated NO release rate of $0.418 \pm 0.18 \mathrm{pmol} / \mathrm{cm}^{2} \mathrm{~s}$.

To assess NO consumption by RBCs, measurements of the rate of NO consumption in a gas stream flowing on top of an RBC solution of specified Hematocrit (Hct) was performed. The consumption rate constant $\left(\mathrm{k}_{\mathrm{bl}}\right)$ in porcine $\mathrm{RBCs}$ at $25^{\circ} \mathrm{C}$ and $45 \%$ Hct was estimated to be $3500 \pm 700 \mathrm{~s}^{-1}$. $\mathrm{k}_{\mathrm{bl}}$ is highly dependent on Hct and can reach up to $9900 \pm 4000 \mathrm{~s}^{-1}$ for $60 \%$ Hct. The nonlinear dependence of $\mathrm{k}_{\mathrm{bl}}$ on Hct suggests a predominant role for extracellular diffusion in limiting NO uptake.

Further simulations showed a linear relationship between varying NO production rates and $\mathrm{NO}$ availability in the SMCs utilizing the estimated NO consumption rate. The corresponding SMC [NO] level for the average $\mathrm{NO}$ production rate estimated was approximately $15.1 \mathrm{nM}$. With the aid of experimental and theoretical methods we were able to examine the NO paradox and exhibit that endothelial derived NO is able to escape scavenging by RBCs to diffuse to the SMCs. 


\section{TABLE OF CONTENTS}

CHAPTER

PAGE

\section{INTRODUCTION}

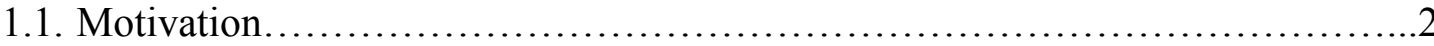

1.2. Background

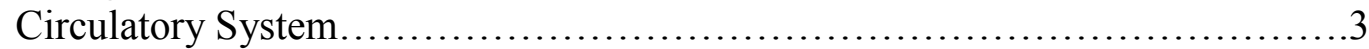

Nitric oxide formation..................................................... 10

Nitric oxide's chemical properties........................................ 11

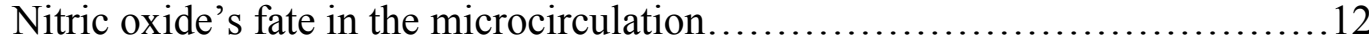

1.3. Detection methods

Chemiluminescence method................................................. 14

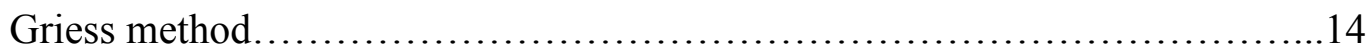

Nitric oxide sensitive electrode sensors...................................14

Fluorometric detection....................................................

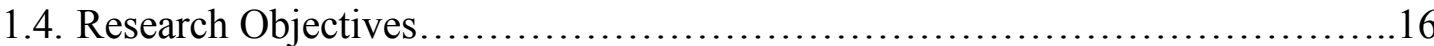

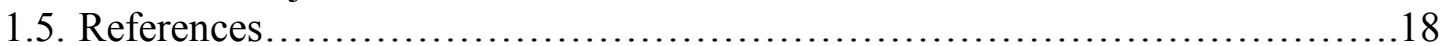

2. KINETIC ANALYSIS OF DAF-FM ACTIVATION BY NO: TOWARD CALIBRATION OF A NO-SENSITIVE FLUORESCENT DYE

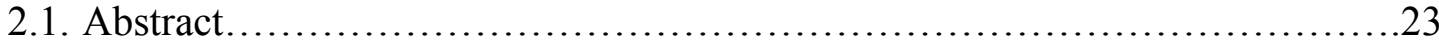

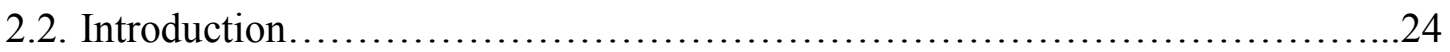

2.3. Methods

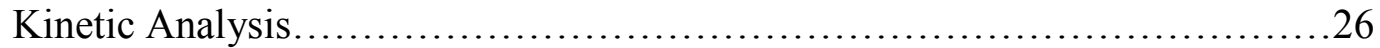

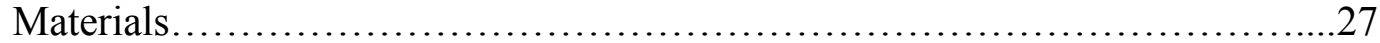

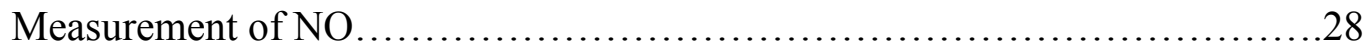

2.4. Results

The "Clamped NO" protocol...........................................28

Dependence of fluorescence intensity of DAF and NO concentrations............30

Fluorescent intensity vs. NO donor......................................... 31

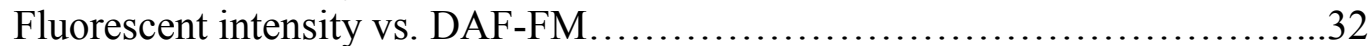

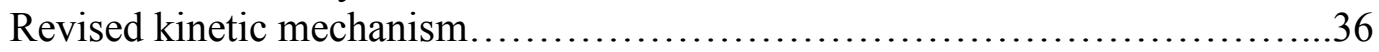

2.5. Discussion

Active intermediate of DAF activation...................................... 38

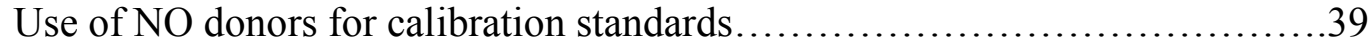

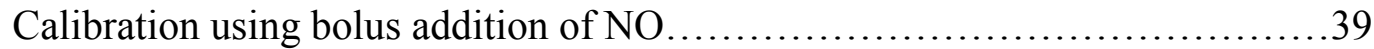

Kinetics of DAF activation..................................................... 40

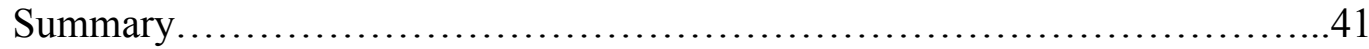

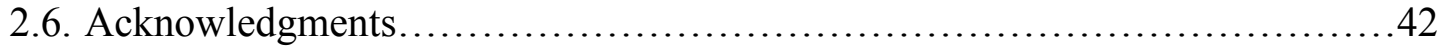

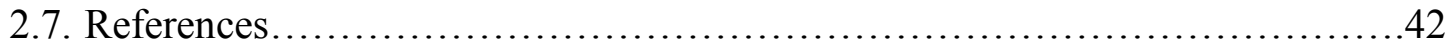

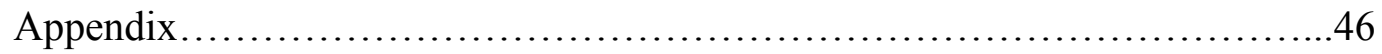


3. APPROXIMATION OF NITRIC OXIDE RELEASE BY ENDOTHELIAL CELLS UTILIZING DAF-FM

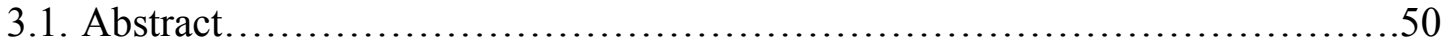

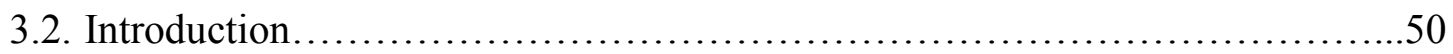

3.3. Methods

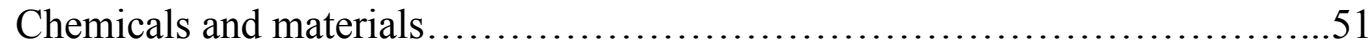

Cell culturing and cell experiment preparation.............................53

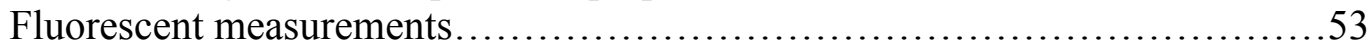

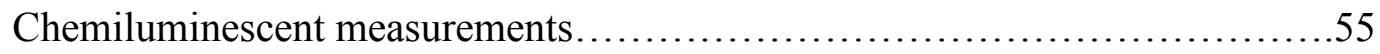

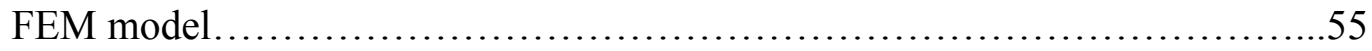

3.4. Results

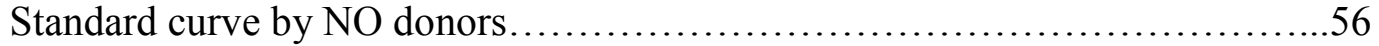

Agonist stimulated fluorescent detection.................................58

Agonist stimulated chemiluminescent detection..............................59

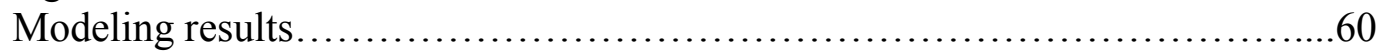

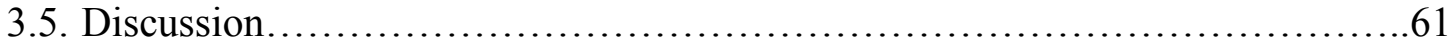

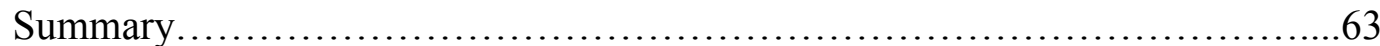

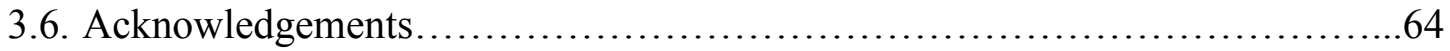

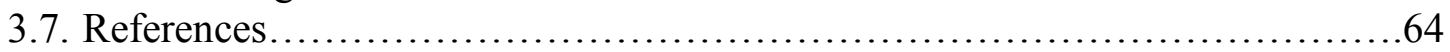

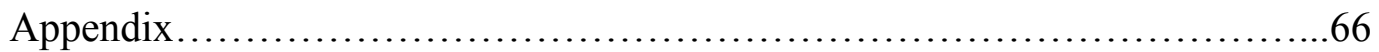

4. THEORETICAL AND EXPERIMENTAL DETERMINATION OF NITRIC OXIDE CONSUMPTION BY RED BLOOD CELLS

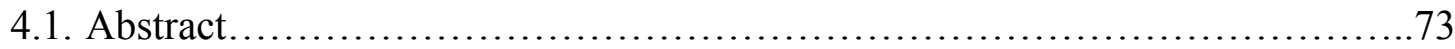

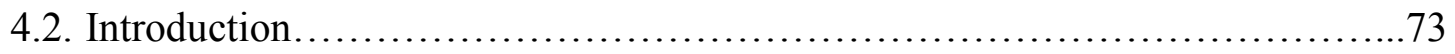

4.3. Methods

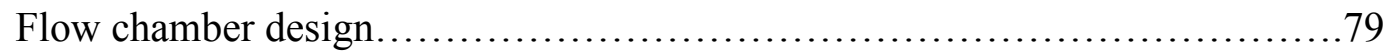

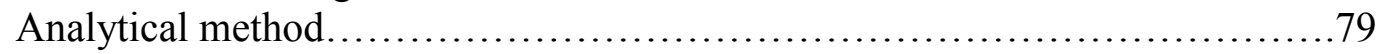

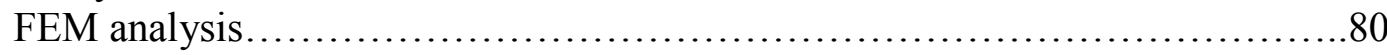

Flow chamber preparation and data acquisition............................. 80

Blood collection and separation............................................ 81

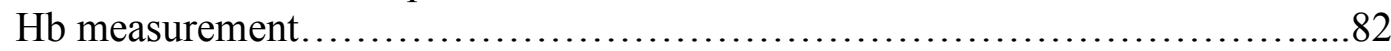

4.4. Results

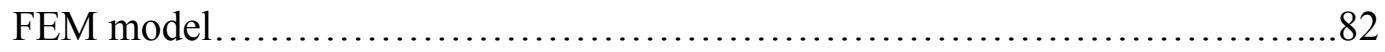

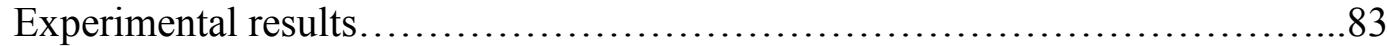

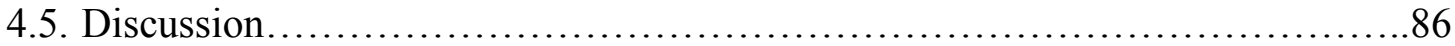

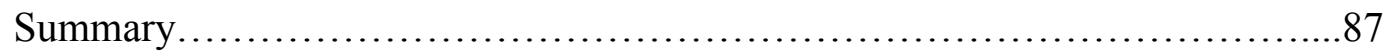

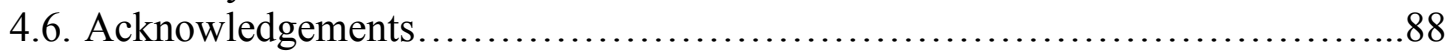

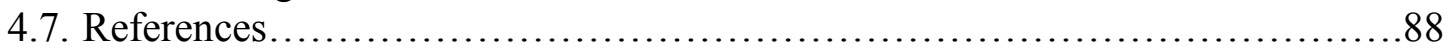

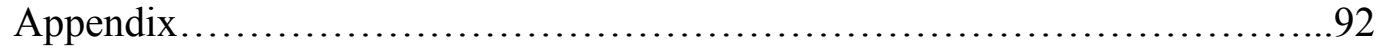

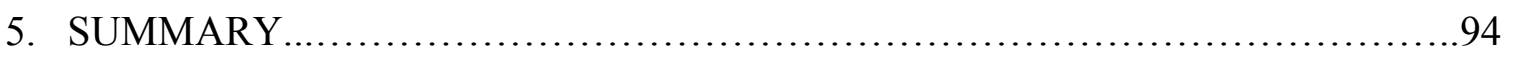

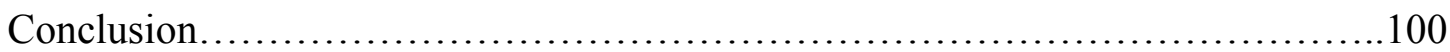

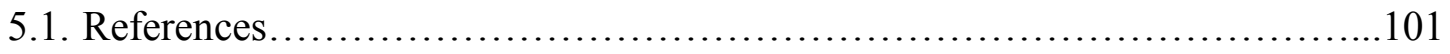

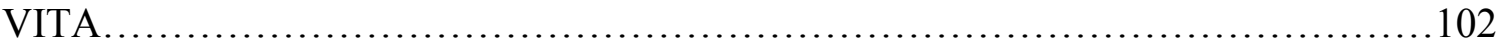




\section{LIST OF TABLES}

TABLE

PAGE

1.1 Endothelial NO production rates $\left(\mathrm{Q}_{\mathrm{NO}}\right)$ previously reported based on a cell volume of $400 \mu \mathrm{m}^{3}$, endothelial layer thickness $4 \mu \mathrm{m}$, and [eNOS] of $0.097 \mu \mathrm{M} \ldots \ldots \ldots \ldots \ldots . . . .11$

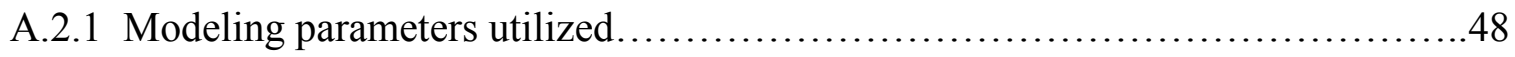

3.1 Individual components and concentrations of premade buffer solution..............53

$3.2 \beta_{\max }$ and corresponding [NO] from HUVECs due to agonist stimulation............63

A.4.1 Chamber parameters and values.....................................93 


\section{LIST OF FIGURES}

FIGURES

PAGE

1.1 Schematic representation of the anatomical structures of the microcirculation........13

1.2 Chemical structure of the cell permeable DAF-FM diacetate and its further reaction

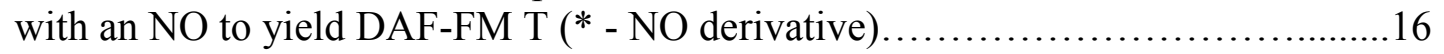

2.1 (A) Predicted continuous release by 1000,500 and $10 \mu \mathrm{M}$ SPER/NO. Maximum NO release is indicated by the dot with a constant increase until a pseudo steady-state plateau is reached. (B) NO release from $500 \mu \mathrm{M}$ SPER/NO over time, detected by an NO electrode $(n=4)$. The dot indicates when maximum [NO] achieved. (B-Insert) Log-log plot displays the relationship between [NO] and [SPER/NO] using maximum [NO] from ranging [SPER/NO] detected by the electrode. The linear fit of the log$\log$ plot has a slope of 0.508 with a standard deviation of 0.208 . The slope is not significantly different than $0.5(\mathrm{p}=0.95)$. 29

2.2 Fluorecent intensity curves obtained from solutions containing $5 \mu \mathrm{M}$ DAF-FM and $50 \mu \mathrm{M}$ SPER/NO (A) simulation (B) experimental, indicating when $\beta_{\max }$ is achieved. 31

2.3 (A) Fluorescent intensity curves showing the dependence of [SPER/NO] $(10 \mu \mathrm{M}$, $100 \mu \mathrm{M}$, and $1 \mathrm{mM}$ ) on slope $\left(\beta_{\max }\right)$. (B) Log-log plot of $\beta_{\max }$ and $[\mathrm{SPER} / \mathrm{NO}](\mathrm{n}=5)$. A linear relationship is observed having a slope of 0.99 with a standard deviation of

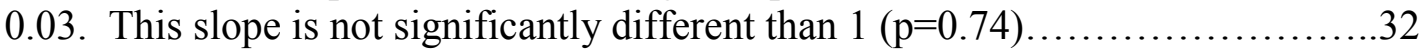

2.4 (A) Data fitting for $\beta_{\max }$ with respect to [DAF-FM] at 12.5 and $125 \mu \mathrm{M}$ [SPER/NO] $(\mathrm{n}=5)$ indicating $\mathrm{K}_{\mathrm{m}}$ values of 1.0 and 5.3 respectively. (B) Log-log plot of $\mathrm{K}_{\mathrm{m}}$ and [SPER/NO] $(\mathrm{n}=5)$ with a linear relationship having a slope of 0.59 and a standard deviation of 0.06 . The slope of this line is statistically different than $1\left(\mathrm{p}<5.5 \times 10^{-5}\right)$

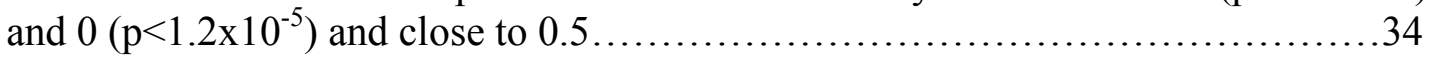

2.5 Relationship of the dependence of $\mathrm{K}_{\mathrm{m}}$ on [NO]. The linear slope of the line yields

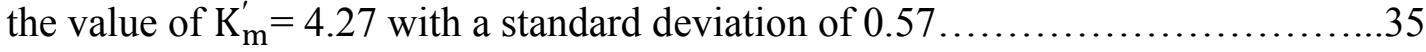

3.1 Data from standard curve experiments $(n=4)$ presented as log-log plots of [SPER/NO] and $\beta_{\max }$. (A) Values from smaller [SPER/NO] ranging from $5-25$ $\mu \mathrm{M}$. Linear fit yields a slope of $1.1 \pm 0.3$. (B) Values from larger [SPER/NO] ranging from $100-750 \mu \mathrm{M}$. Linear fit yields a slope of $0.4 \pm 0.2 \ldots \ldots \ldots \ldots \ldots . . .57$

3.2 Estimated $\beta_{\max }$ values obtained from HUVECs after agonist stimulation, with and

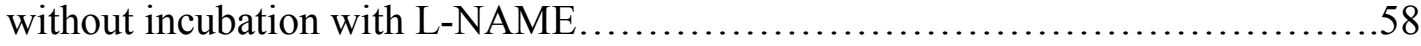

3.3 Chemiluminescent data from HUVECs after agonist stimulation. (A) Overall data shown in comparison to control without agonist stimulation (B) Normalized [NO]

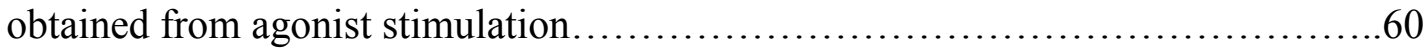


3.4 FEM analysis results. (A) A very fine mesh was utilized for the investigation area yielding (B) NO concentration levels at a production rate of $1.0 \mathrm{pmol} / \mathrm{cm}^{2} \mathrm{~s}$. Maximum NO levels achieved at this production rate was $616.9 \mathrm{nM} \ldots \ldots \ldots \ldots \ldots . . .61$

3.5 Standard calibration data fitted to modeled equation to enable estimations of [NO] from endogenously produced sources........................................62

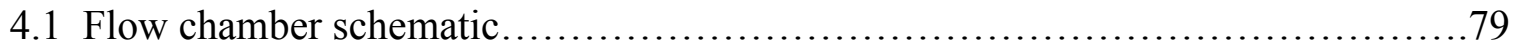

4.2 Schematic of experimental flow chamber design.................................. 81

4.3 FEMLAB and analytical solution comparison................................... 83

4.4 Previous model predictions for the observed first order reaction rate constant of NO

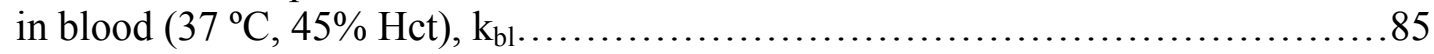

4.5 Updated model predictions of $\mathrm{k}_{\mathrm{bl}}$ at $25{ }^{\circ} \mathrm{C}$ including experimental data............86

5.1 NO concentration levels available in the SMCs assuming an endothelial derived NO production rate of $1.0 \mathrm{pmol} / \mathrm{cm}^{2} \mathrm{~s}$ and an $\mathrm{NO}$ consumption rate of $3500 \mathrm{~s}^{-1} \ldots \ldots \ldots . .97$

5.2 Linear relationship between varying $\mathrm{NO}$ production rates and a SMCs NO concentration levels simulated by theoretical arteriole model assuming a constant

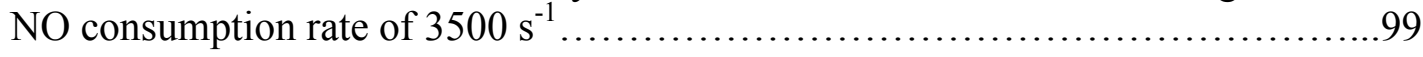

5.3 Summarized data of simulations performed for varying combinations of NO

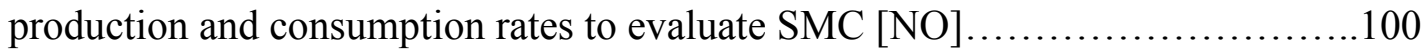




\section{LIST OF ACRONYMS}

4-Amino-5-Methylamino-2',7'-Difluorescein

DAF-FM

Benzotriazole

$\mathrm{T}$

Calmodulin

$\mathrm{CaM}$

Calcium

$\mathrm{Ca}^{2+}$

Calcium Chloride

$\mathrm{CaCl}$

Carbon Dioxide

$\mathrm{CO}_{2}$

Diacetate

da

Diffusion Area

S

Dimethyl Sulfoxide Anhydrous

DMSO

Cyclic Guanosine Monophosphate

cGMP

Endoplasmic Reticulum

ER

Endothelial Cells

EC

Endothelial Derived Hyperpolarizing Factor

EDHF

Endothelium Derived Relaxing Factor

EDRF

Endothelium Nitric Oxide Synthase

eNOS

Fetal Bovine Serum

FBS

Fluorescent Intensity

FI

Flux

Guanosine Triphosphate

GTP

Height

$\mathrm{H}$

Hematocrit

Hct

Hemoglobin

$\mathrm{Hb}$ 
Nitrate

Nitric Oxide Analyzer NOA

Nitric Oxide Synthase

NOS

Nitrite $\mathrm{NO}_{2}$

Nitrosothiols RSNO

$\mathrm{N}_{\omega}$-Nitro-L-Arginine Methyl Ester Hydrochloride

Oxygen

Potassium Chloride

Potassium Phosphate $\mathrm{KH}_{2} \mathrm{PO}_{4}$

Pseudo Steady State Approximation PSSA

Red Blood Cells

$\mathrm{RBC}$

Sodium Carbonate $\mathrm{NaHCO}_{3}$

Sodium Chloride $\mathrm{NaCl}$ 
Sodium Hydroxide

$\mathrm{NaOH}$

Sodium Nitrite

$\mathrm{NaNO}_{2}$

Soluble Guanylate Cyclase

sGC

Smooth Muscle Cells

SMC

Spermine NONOate

SPER/NO

Tetrahydrobioprotein

$\mathrm{BH}_{4}$

Velocity

v

White Blood Cells

WBC

Width

W 
Chapter 1

INTRODUCTION 


\subsection{Motivation}

The 2012 Statistical Fact Sheet update on high blood pressure prevalence by the American Heart Association stated that 76.4 million people in the United States over the age of 20 have high blood pressure or hypertension. This approximates to about 1 in every 3 adults. Future projections estimate that by the year 2030, an additional 27 million more people will be affected, a 9.9\% increase from the estimates performed in 2010. In addition, in 2008, estimated direct and indirect costs of high blood pressure were $\$ 50.6$ billion [1]. Since there are no physical symptoms, many people are unaware that they are affected. Data from 2005-2008 showed that $79.6 \%$ of people with high blood pressure are unaware they have it. However, high blood pressure is a leading cause of heart attack, stroke, and congestive heart failure. Statistics show that $69 \%$ of patients that experience their first heart attack, $77 \%$ of patients that experience their first stroke, and $74 \%$ of patients who are diagnosed with congestive heart failure, have blood pressures higher than 140/90 $\mathrm{mmHg}$ [1]. Blood pressure is responsible for the maintenance of blood flow in the circulatory system. Regulation of blood pressure is attained by vessel constriction and dilation [2]. Understanding the nature of vessel reactivity is important in further understanding the clinical implications of hypertension.

In 1987, Nitric Oxide (NO) was identified as the Endothelium Derived Relaxing Factor (EDRF). EDRF was known as the agent involved in inducing relaxation of the smooth muscle cells (SMC) causing vasodilation. Prior to this discovery, NO was thought to be a toxic environmental pollutant. However, upon realizing that NO exhibited these vasodilating effects, researchers worked to find other physiological and 
pathophsyiological areas in which NO played a role. There have been over 100,000 scientific papers published that focus on NO in multiple facets of research, most of which correlates to work performed within the last 5 years [3]. It has been revealed that NO plays a part in numerous physiological processes such as angiogenesis, reproduction, inflammation, neurotransmission, platelet and leukocyte adhesion, host defense response, apoptosis, and the regulation of vascular tone and blood flow. In addition, NO is also involved in many pathological conditions such as septic shock, atherosclerosis, ischemia/reperfusion injury, and carcinogenesis $[4,5]$.

The recent understanding of NO in physiological processes has aided in the development of pharmacological drugs and treatments. Examples include administration of gaseous NO for treatment of respiratory failure, intravenous administration of NO donors for treatment of hypertension, and oral administration of medication affecting NO production for treatment of erectile dysfunction. Although there have been great strides in discovering functional roles of NO physiologically, a greater understanding of NO production and NO pathways would facilitate progression in pharmacological advancements in treating ailments such as hypertension.

\subsection{Background}

Circulatory system. The circulatory system, in conjunction with the heart and blood vessels, is the basis for the cardiovascular system. The primary function of this system is the transport of necessary nutrients to all parts of the body. Blood flowing through the cardiovascular system can be divided into two pathways, the pulmonary and systemic pathways. These two pathways work together to oxygenate blood flowing 
through the vessels and deliver the oxygenated blood to every part in the body. Deoxygenated blood from the systemic pathway enters the heart in the right atrium, where the right ventricle then pumps this blood into the pulmonary pathway, which passes through the lungs. In the lungs, the blood becomes oxygenated. The freshly oxygenated blood then enters the left atrium ending the pulmonary pathway. The right ventricle then pumps the blood, via the systemic pathway, enabling delivery of oxygen and nutrients to the whole body [6].

The vasculatures in both pathways are important components of the circulatory system and can be classified into three main categories: arteries, veins, and capillaries. Arteries are blood vessels that carry blood away from the heart. The blood flowing through the arteries of the pulmonary system is deoxygenated, whereas the blood in the arteries of the systemic system is oxygenated. Contrastingly, veins are blood vessels that return blood to the heart. Therefore, the veins of the pulmonary system are oxygenated and the veins of the systemic system are deoxygenated. The third category of blood vessels is the capillaries. These vessels are tiny thin-walled vessels that connect the arteries and veins. This is also the site of the exchange of oxygen and nutrients for metabolic wastes, heat, and carbon dioxide [6].

The anatomy of blood vessels can be described with three layers: tunica interna, tunica media, and tunica externa. The tunica interna is the internal layer of the vessel wall and is comprised of a single layer of endothelial cells (EC). The ECs play an important role in regulating blood pressure as well as absorbing nutrients from the blood flowing in close proximity. Immediately adjacent to the tunica interna is the tunica 
media, and as the name suggests, is the middle layer of the vessel. This layer is composed of concentric sheets of vascular SMCs and is the thickest layer of the vessel. When the SMCs contract, the vessel diameter decreases, thus increasing the blood pressure within the lumen of the vessel. On the other hand, when the SMCs dilate, the vessel diameter increases, causing the blood pressure to reduce. A variety of chemicals can produce changes to the state of the SMCs including neurotransmitters, hormones, and paracrines [6]. The outer layer of the vessel, the tunica externa, is mainly composed of connective tissue, which allows connection to surrounding tissues [2].

The difference between arteries and veins, other than the oxygenated state of the blood flowing within, are the wall thicknesses. Arteries tend to have a greater mean wall thickness, almost double the value of most veins [6]. The thickness is mostly associated to a thicker tunica media. The natural flexibility of the SMCs yields the arteries as a whole to be more flexible. This characteristic is ideal and will allow arteries to resist the high pressures of blood flow generated by the heart. Arteries also have a slightly smaller mean diameter than veins. In addition, the amount of veins out numbers the amount of arteries. Combining this fact with the overall larger diameter than arteries, veins as a result hold more than half of the blood in the circulatory system [6].

Arteries can be further examined and classified into three categories: elastic arteries, muscular arteries, and arterioles. The elastic arteries are the first arteries to branch from the heart and are immediately affected by the great pressures generated by the heart. The most important function of this type of artery is its ability in controlling the pressure within the vessel. When the ventricles of the heart pumps the blood into the 
elastic arteries, known as ventricular systole, the arteries respond by increasing in size to adapt to the large amounts of blood present. This event is followed by ventricular diastole, in which the elastic arteries respond by reducing in size. The expansion and compression of the elastic arteries during these two events ensure constant blood flow circulation [2]. The second category of arteries is known as the muscular arteries. The role of these arteries are to provide oxygen and nutrient rich blood to surrounding muscles and internal organs. In addition, muscular arteries play a role in regulating blood flow. The last category of arteries is the arterioles. These are the smallest types of arteries, with diameters in the micron range. Arterioles have a high content of SMCs and have the ability to contract and dilate according to surrounding signals. Because of their small diameters, arterioles are sites of high resistance in blood flow, especially when they are stimulated to constrict [6].

Veins can also be divided into three categories: large veins, medium-sized veins, and venules. The large veins are known as the superior and inferior venae cavae. As mentioned before, veins are the vessels that return blood back to the heart. The superior and inferior venae cavae are the largest veins that deliver the deoxygenated blood from the systemic system back to the heart. The medium-sized veins are smaller than the large veins, but the overall wall thickness is not that great. This is because they are not subject to high pressures like that of the arteries. The last type of veins is the venules. These are the smallest of the veins and are responsible for receiving the blood leaving the capillaries. Venules contain little to no SMCs and are composed primarily of ECs and fibrous tissue [6]. 
The capillaries are the blood vessels that connect the arteries and veins. They are the smallest vessels in the circulatory system. The diameter of a capillary is approximately $8 \mu \mathrm{m}$, which is the approximate size of a single red blood cell (RBC). As it was previously mentioned, the capillaries are the site of exchange of oxygen and nutrients for wastes and carbon dioxide [6]. The capillaries, in combination with the arterioles and the venules, compose the microcirculation.

Blood pressure is the driving force for blood flow; therefore it needs to be regulated carefully. This is accomplished by monitoring blood volume, cardiac output, peripheral resistance, and relative distance of blood between arterial and venous blood vessels. Although the volume of blood within the circulatory system is relatively constant, for the exceptions of trauma where there is loss of blood, slight changes in blood volume may still have an effect on blood pressure. Increases in blood volume occur constantly with the ingestion of food and water. The kidneys are responsible for regulating increased blood volume by excreting excess as urine. In the event that there is a decrease in blood volume, the only way to increase it is either by drinking more fluids or via intravenous infusions [6].

Cardiac output must also be monitored in regulating blood pressure. Cardiac output gives information as to the effectiveness of the heart and its pumping abilities. To determine cardiac output, information about heart rate and stroke volume is needed. Heart rate, as the name suggests, is the rate at which the heart is beating. Stroke volume is the amount of blood pumped by one ventricle [6]. To calculate cardiac output, the 
heart rate is multiplied by the stroke volume. Therefore, the effectiveness of the heart pumping is measured by volume of blood pumped per ventricle per time.

The third aspect of blood pressure that needs monitoring is the peripheral resistance. Peripheral resistance is mainly due to the resistance produced in the arterioles. Arteriole resistance contributes to approximately $60 \%$ of the total systemic resistance [6]. As previously mentioned, the small diameters provide high resistance to blood flow, especially when in a constricted state. Therefore, the resistance to blood flow is proportional to the length of the arteriole and the viscosity of the fluid, and inversely proportional to the radius of the arteriole to the fourth power. Hence, if the diameter of the arteriole decreases, caused by constriction, the resistance increases [6].

The final aspect that affects blood pressure is the relative distribution of blood between arterial and venous blood vessels. As mentioned, veins contain more than half of the total blood supply due to their abundance and larger mean diameter in comparison to arteries. In order to monitor the distribution of blood, the diameter of the veins must be examined [6]. In the event that the diameter of the veins decrease, the total amount of blood within the veins would decrease as well, altering the distribution of oxygenated to deoxygenated blood within the circulatory system.

There are also other means of regulating blood pressure. Vascular SMC have the ability to regulate blood pressure by their state of contraction. This ability is known as myogenic autoregulation and occurs when SMCs contract in direct response to being stretched by increased pressure [6]. Local regulation can also be achieved by secreted paracrines. Such paracrines include oxygen $\left(\mathrm{O}_{2}\right)$, carbon dioxide $\left(\mathrm{CO}_{2}\right)$, and NO. Their 
presence or absence as well as their concentrations will dictate the vascular state, and ultimately blood pressure. For example, in the event that there is a low $\mathrm{O}_{2}$ supply, and in response a high $\mathrm{CO}_{2}$ concentration, vasodilation will occur to increase the blood supply to the region in need of oxygen exchange [2]. Endothelium mediated regulation, as the name suggests, occurs when endothelial produced signals change the contracted state of a vessel. The endothelium can produce signals in various ways. One way is in response to the shear stresses experienced by the blood flowing through the lumen of the vessel. Another way is in response to agonists. Typical signaling molecules produced by the endothelium are NO, prostaglandins, and the endothelium-derived hyperpolarizing factor (EDHF) [2].

Another important component of the circulatory system is the blood that is traveling within it. Blood makes up $1 / 4$ of the extracellular fluid or internal environment. It is composed of two parts: the plasma and the cellular elements. The plasma is the fluid portion of the blood with water being the main component. Within the plasma are ions, organic molecules such amino acids and proteins, vitamins, and gases such as $\mathrm{O}_{2}$ and $\mathrm{CO}_{2}$. The cellular elements of the blood include white blood cells (WBC), platelets, and RBCs. WBCs play a role in protecting the body from foreign bodies. More specifically, this task is performed by lymphocytes, monocytes, neutrophils, eosinophils, and basophils [6]. Platelets are the second cellular element within the blood and they assist in forming clots needed during the coagulation process.

RBCs make up the majority of cellular elements in the blood. The amount of RBCs within the blood is known as the hematocrit (Hct). Average Hct of an adult male is 
approximately $40-54 \%$, and the average Hct of an adult woman is $37-47 \%$ [6]. The primary role of RBCs, as discussed before, is to transport nutrients and oxygen to sites of need, and to remove wastes in exchange. Hemoglobin $(\mathrm{Hb})$ is a heme-containing protein that occupies $95 \%$ of RBCs. $\mathrm{Hb}$ is the actual transporting mechanism of gases in the blood. The oxidative state of blood depends on the oxidative state of the $\mathrm{Hb}$. Deoxy-Hb means there is no oxygen attached, whereas oxy- $\mathrm{Hb}$ infers that there is oxygen bound to the $\mathrm{Hb}$.

Nitric oxide formation. The inner lining ECs of the vasculature are the sites of NO production. NO is produced endogenously by the enzyme nitric oxide synthase (NOS). There are several isoforms of this enzyme including neuronal NOS (nNOS), inducible NOS (iNOS), and endothelium NOS (eNOS). iNOS is only produced during an inflammatory response whereas nNOS and eNOS are produced constitutively in the cells. When examining NO production in the vasculature, eNOS is the isoform focused on. Hemodynamic or agonist stimuli activate receptors on the membrane of the ECs causing an increase in eNOS production. Agonist stimuli include, but are not limited to acetylcholine, bradykinin, and insulin. Upon agonist stimulation and/or experiences of mechanical forces, there is an increase in $\mathrm{Ca}^{2+}$ entry into the ECs via channels. This in turn causes an increase in the concentration of intracellular inositol triphosphate $\left(\mathrm{IP}_{3}\right)$, which initiates the release of stored calcium $\left(\mathrm{Ca}^{2+}\right)$ from the endoplasmic reticulum (ER). The $\mathrm{Ca}^{2+}$ then binds to calmodulin $(\mathrm{CaM})$ to activate the eNOS. Other cofactors needed to activate this enzyme are tetrahydrobioprotein $\left(\mathrm{BH}_{4}\right)$, nicotinamide-adeninedinucleotide phosphate (NADPH), and $\mathrm{O}_{2}$. Once the eNOS is activated, it then catalyzes 
the reaction that yields NO and L-citrulline via a five electron oxidation of the guanidinenitrogen terminal of L-arginine [7, 8]. Actual endothelial derived NO production rates have been estimated to range by many orders of magnitude. Table 1.1 shows a list of production rates reported.

\begin{tabular}{|c|l|}
\hline $\begin{array}{c}\text { Q } \\
0.003-0.034 \\
\mu \mathrm{Ms}^{-1}\end{array}$ & $\begin{array}{l}\text { Modeling of pathways of eNOS for NO and superoxide production } \\
\text { rates [9] }\end{array}$ \\
\hline $\begin{array}{c}2.19 \\
\mathrm{pMs}^{-1}\end{array}$ & $\begin{array}{l}\text { In vitro experiments from BAEC following stimulation by } \\
\text { Ethinylestradiol [10] }\end{array}$ \\
\hline 415 & $\begin{array}{l}\text { In vitro experiments from HUVEC following stimulation by VEGF } \\
{[11]}\end{array}$ \\
\hline $\mathrm{nMs}^{-1}$ & $\begin{array}{l}\text { In vitro experiments from HUVEC upon flow-induced stimulation } \\
\mathrm{nMs}^{-1}\end{array}$ \\
\hline 2.117
\end{tabular}

Table 1.1 - Endothelial NO production rates $\left(\mathrm{Q}_{\mathrm{NO}}\right)$ previously reported based on a cell volume of $400 \mu \mathrm{m}^{3}$, endothelial layer thickness $4 \mu \mathrm{m}$, and [eNOS] of $0.097 \mu \mathrm{M}$.

There can be several reasons for the large range of estimated NO production rate values. Especially in modeling circumstances, incorrect assumptions of cell size and volume can generate incorrect production rate values. Also, the NO detected in experimental scenarios could be contributed from other sources, leading to a value of NO larger than what was actually endogenously produced. In addition, the method in which the production rate is determined could be a factor in the differences in reported NO production rate values, given that some detection systems are more sensitive than others.

Nitric oxide's chemical properties. NO is an uncharged diatomic molecule and can diffuse freely to neighboring cells, allowing it to perform in both an autocrine and paracrine fashion. NO is also a free radical making it a highly reactive species with a short half-life. Reactions that have shown to dominate the fate of $\mathrm{NO}$ in the 
microcirculation are those with superoxides, $\mathrm{Hb}$, myoglobin, guanylate cyclase, and cytochrome c oxidase $[7,8,14]$. NO also reacts with $\mathrm{O}_{2} \cdot \mathrm{O}_{2}$ 's biradical characteristics make it a good candidate in reacting with free radicals. Because $\mathrm{O}_{2}$ has two unpaired electrons, and NO has only one unpaired electron, the product of the two will yield a form with one unpaired electron, $\mathrm{NO}_{2}$ radical. This species is quite unstable and can easily react further [15].

Nitric oxide's fate in the microcirculation. Endothelial derived NO once produced, may diffuse to the adjacent SMCs where it activates the hemoprotien soluble guanylate cyclase (sGC) by removing the histidine residue on its axial position. sGC then catalyzes the conversion of guanosine triphosphate (GTP) to cyclic guanosine monophosphate (sGMP), thus causing relaxation of the SMCs [8]. However, as previously explained, NO reacts quickly with heme-containing proteins. The close proximity of the ECs, the site of NO production, to the lumen of the vasculature, yields a second pathway that endothelial derived NO may pass. The lumen consists of RBCs containing both oxy- and deoxy-Hb. Due to NO's reactive nature, it is suggested that a significant amount of endothelium derived $\mathrm{NO}$ will react with either form of $\mathrm{Hb}$. Although NO is known to induce vasodilation, it is still unclear how it is able to diffuse to the SMCs and sustain physiological relevant concentrations to induce vasorelaxation, without being scavenged by the RBCs. This phenomenon is referred to as the "NO Paradox" [14, 16-19]. Figure 1.1 below displays a schematic of an anatomical representation of the location of the ECs with respect to the SMCs and the RBCs. 


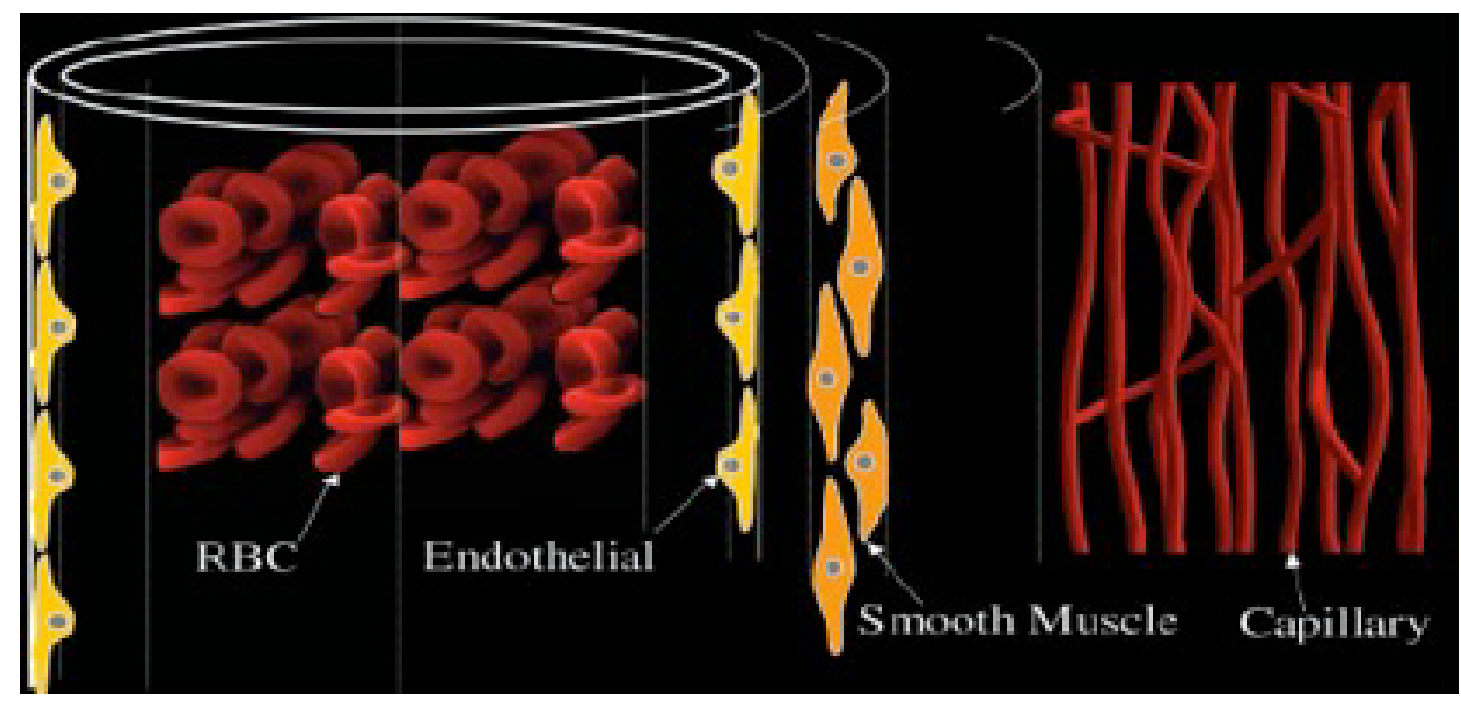

Figure 1.1 - Schematic representation of the anatomical structures of the microcirculation [20].

There have been several experimental and theoretical studies performed to explain the fate of NO within the microcirculation, proposing that NO may encounter limitations in its reaction with oxy- and deoxy-Hb either via $\mathrm{RBC}$ membrane resistance, or limited by extracellular diffusion. It has also been suggested that NO can also be preserved. These hypotheses propose mechanisms that regulate NO's bioavailability [19-25]. On the whole, parameter values as well as a final conclusion regarding what actually prevents NO uptake by the RBCs has yet to be elucidated. This information would be essential in understanding more about NO's role as a vasodilator.

\subsection{Detection methods}

There are various methods available for detecting NO. Some methods merely detect the presence of $\mathrm{NO}$, whereas other methods can determine NO concentrations ([NO]). All existing methods vary according to their sensitivities and specificities to NO. 
Chemiluminescence method. Chemiluminescence is considered one of the most sensitive assay systems for the detection of NO. It generally can detect any radical at low concentrations in real-time. It has been noted that physiological levels of endogenously produced NO range from the pico- to nanomolar ranges, therefore this system has the ability to detect these low concentrations [26]. Drawbacks to this method are its inability to detect intracellular [NO], because only the supernatant fluid of a cellular system can be analyzed. In addition, chemiluminescence cannot give real-time output. Lastly, this assay cannot be performed in the presence of $\mathrm{Hb}$ [27].

Griess method. Although this method is used to detect NO, it does not measure NO directly. Rather, the measurement of the stable oxidation product nitrite is detected. This product is formed with the rapid interaction between $\mathrm{NO}$ and $\mathrm{O}_{2}$. The Griess reaction is performed utilizing a two-step diazotiation reaction, in which under acidic conditions, the nitrite produces a diazonium ion. This ion is further processed to form a chromophoric azo derivative, which can be monitored using spectrophotometric measurements [27]. The detection limits for this assay system is not that sensitive with a range between $0.1-1.0 \mu \mathrm{M}$.

Nitric oxide sensitive electrode sensors. Electrodes sensitive to NO can be employed as a method for NO detection. One benefit of electrodes is its ability to provide real-time detection of NO. The drawback of this method however is its lack of ability to yield information about the spatial distribution of NO. The amount of NO detected by the electrode is determined by the amount of NO sensed only at the electrode tip [28]. 
Fluorometric detection. Fluorometric analysis is the only method available for intracellular NO studies [29]. The development of fluorescent probes in the recent years has demonstrated to be a sensitive and specific assay system that is gaining wide application to measure nitrogen oxides [29-35]. NO-reactive fluorescent indicators allow bio-imaging of $\mathrm{NO}$ with high spatiotemporal resolution, permitting investigation of twodimensional NO production in real-time by digital fluorescence imaging, which is not possible with the other NO detection methods [36]. These fluorescent probes may be used to measure nanomolar levels of NO generated under biological conditions [37]. One such indicator is $\mathrm{Cu}$ (II) fluorescein-based compound, $\mathrm{CuFL}(1)$. This fairly new probe has been shown to be specific for NO detection. Fluorescence is achieved with NO under both anaerobic and aerobic conditions, indicating that it reacts directly with $\mathrm{NO}$ as opposed to an oxidative nitrogen species [34].

One of the most widely used fluorescent NO detection probes is, 4-amino-5methylamino-2',7'-difluorescein (DAF-FM). DAF-FM is virtually non-fluorescent, however, upon reaction with NO, a highly fluorescent benzotriazole is formed (DAF-FM T). Because the reaction between NO and DAF-FM is irreversible, the fluorescence intensity (FI) will not decrease. DAF-FM T exemplifies greater stable photobleaching effects than its prior fluorescent indicators, is shown to be more stable at a $\mathrm{pH}$ above 5.8, and is more sensitive with detection limits up to $3 \mathrm{nM}$ [27]. Figure 1.2 below shows the chemical structure of DAF-FM and its reaction scheme. 


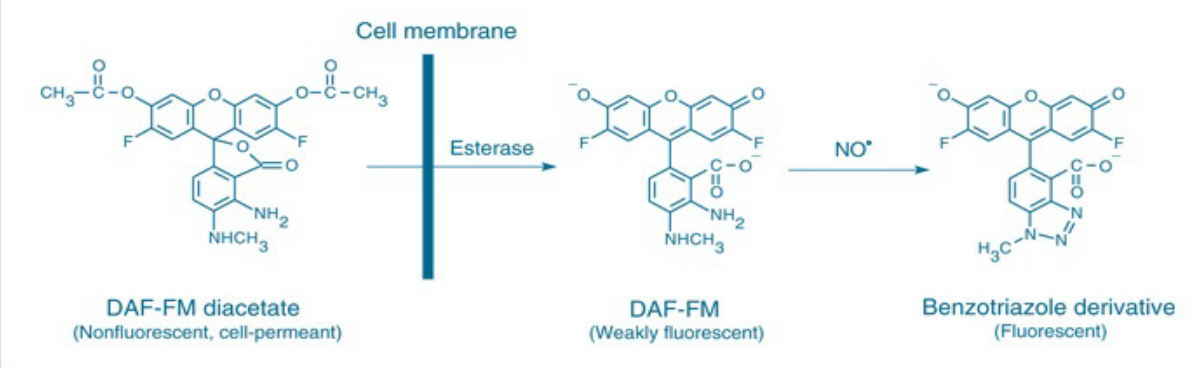

Figure 1.2 - Chemical structure of the cell permeable DAF-FM diacetate and its further reaction with an NO to yield DAF-FM T (* - NO derivative) [38].

\subsection{Research Objectives}

The ability to understand the fate of NO within the vasculature is limited due to inconsistent reported data with respect to both production and consumption rates. The measurement of $[\mathrm{NO}]$ and production rates in biological tissues is difficult due to its short half-life, however different methods have been attempted. Recently, the use of NO sensitive fluorescent indicators has gained interest due its promising spatiotemporal resolution. One such indicator is DAF-FM. DAF-FM has been shown to react with NO or NO derived products to produce a fluorescent signal [36]. Unlike many fluorescent indicators, DAF-FM is not a ratiometric dye; therefore, calibration has been difficult due to factors such as dye leakage, uneven dye loading, and photobleaching [39, 40]. More importantly, the actual kinetics of the reaction between NO and DAF-FM has yet to be elucidated. Calibration of this dye can be beneficial in estimating [NO] as well as determining NO production rates.

The focus of this dissertation was to yield estimations of both production and consumption rates of NO. Specifically, the following objectives were accomplished: 
- Development of a mathematical model that simulates DAF-FM activation with NO upon formulation of NO/DAF-FM reaction kinetics.

- Validation of the developed mathematical model with experimental studies of NO/DAF-FM reactivity in solution.

- Development of a calibration procedure to quantify NO production rates by ECs utilizing DAF-FM upon agonist stimulation.

- Estimation of NO reactivity with RBCs flowing in a flow chamber by examining the effects of Hct on NO consumption.

- Determine NO bioavailability within the SMCs incorporating the derived NO production and consumption rates into a mathematical model.

The first three objectives are focused on studying the endothelial derived NO production rates. The NO-sensitive fluorescent dye, DAF-FM, was utilized in examining the production of NO. It was hypothesized that incorporating a mathematical model of NO/DAF-FM reaction kinetics, in conjunction with in vitro solution experiments, proper calibration of the dye could be performed. Understanding of this reaction scheme and formation of a proper calibration protocol will allow investigation of NO production by cellular systems. Experiments performed with the DAF-FM and ECs not only test the validity of the developed calibration procedure, but yields information about [NO] and NO production rates.

Similarly, NO consumption rates were studied via both mathematical models and experimental procedures as stated in the fourth objective. It is initially hypothesized that the factor limiting NO uptake by RBCs is via extracellular diffusion. This hypothesis 
was based on a previously designed mathematical model [20]. Determining the relationships between the reaction rates of NO with the RBCs $\left(\mathrm{k}_{\mathrm{bl}}\right)$ at different Hct will give insight into this theory. In addition, an estimation of membrane permeability can be determined from experimental results. Finally, experimental findings of both NO production and consumption rates were used to meet the final objective. These findings were incorporated into a detailed mathematical model to make predictions that provided concentration values SMC NO availability.

\subsection{References}

[1] V. L. Roger, et al., "Heart disease and stroke statistics--2012 update: a report from the American Heart Association," Circulation, vol. 125, pp. e2-e220, Jan 3 2012.

[2] F. H. Martini, Fundamentals of anatomy and physiology. Upper Saddle River: Prentice-Hall, 2001.

[3] (2011). Nitric Oxide Society. Available: www.nitricoxide.ws

[4] J. A. Angus, Cocks, T.M., "Vasodilation and the discovery of endotheliumderived relaxing factor," Med J Aust, vol. 146, pp. 250-253, 1987.

[5] L. J. Ignarro, et al., "Endothelium-derived relaxing factor produced and released from artery and vein is nitric oxide," Proc Natl Acad Sci U S A, vol. 84, pp. 92659, Dec 1987.

[6] D. U. Silverthorn, Human physiology: An integrated approach. San Fransisco: Pearson, 2004.

[7] J. S. Beckman and W. H. Koppenol, "Nitric oxide, superoxide, and peroxynitrite: the good, the bad, and ugly," Am J Physiol, vol. 271, pp. C1424-37, Nov 1996.

[8] A. Ortega Mateo, Amaya Aleixandre de, A., "Nitric oxide reactivity and mechanisms involved in its biological effects," Pharmacol Res, vol. 42, p. 427, 2000 . 
[9] S. Kar and M. Kavdia, "Modeling of biopterin-dependent pathways of eNOS for nitric oxide and superoxide production," Free Radic Biol Med, vol. 51, pp. 141127, Oct 12011.

[10] J. F. Arnal, et. al., "Ethinylestradiol does not enhance the expression of nitric oxide synthase in bovine endothelial cells but increases the release of bioactive nitric oxide by inhibiting superoxide anion production," Proc Natl Acad Sci US $A$, vol. 93, pp. 4108-4113, 1996.

[11] J. D. Hood, et al., "VEGF upregulates ecNOS message, protein, and NO production in human endothelial cells," Am J Physiol, vol. 274, pp. H1054-8, Mar 1998.

[12] M. J. Kuchan and J. A. Frangos, "Role of calcium and calmodulin in flowinduced nitric oxide production in endothelial cells," Am J Physiol, vol. 266, pp. C628-36, Mar 1994.

[13] S. Potdar and M. Kavdia, "NO/peroxynitrite dynamics of high glucose-exposed HUVECs: chemiluminescent measurement and computational model," Microvasc Res, vol. 78, pp. 191-8, Sep 2009.

[14] M. Brunori, "Nitric oxide, cytochrome-c oxidase and myoglobin," Trends Biochem Sci, vol. 26, pp. 21-3, Jan 2001.

[15] L. J. Ignarro, Nitric oxide: Biolgoy and Pathobiology. San Diego: Academic Press, 2000.

[16] A. R. Butler, et al., "Diffusion of nitric oxide and scavenging by blood in the vasculature," Biochim Biophys Acta, vol. 1425, pp. 168-76, Sep 161998.

[17] J. R. Lancaster, Jr., "Simulation of the diffusion and reaction of endogenously produced nitric oxide," Proc Natl Acad Sci U S A, vol. 91, pp. 8137-41, Aug 16 1994.

[18] J. C. Liao, et al., "Intravascular flow decreases erythrocyte consumption of nitric oxide," Proc Natl Acad Sci U S A, vol. 96, pp. 8757-61, Jul 201999.

[19] M. W. Vaughn, et al., "Erythrocyte consumption of nitric oxide: competition experiment and model analysis," Nitric Oxide, vol. 5, pp. 18-31, Feb 2001.

[20] N. M. Tsoukias and A. S. Popel, "Erythrocyte consumption of nitric oxide in presence and absence of plasma-based hemoglobin," Am J Physiol Heart Circ Physiol, vol. 282, pp. H2265-77, Jun 2002.

[21] X. Liu, et al., "Diffusion-limited reaction of free nitric oxide with erythrocytes," $J$ Biol Chem, vol. 273, pp. 18709-13, Jul 241998. 
[22] X. Liu, et al., "Nitric oxide uptake by erythrocytes is primarily limited by extracellular diffusion not membrane resistance," J Biol Chem, vol. 277, pp. 26194-9, Jul 192002.

[23] D. J. Singel, Stamler, J.S.,, "Chemical physiology of blood flow regulation by red blood cells: role of nitric oxide and s-nitrosohemoglobin," Annual Review of Phys, 2004.

[24] N. M. Tsoukias, et al., "A theoretical model of nitric oxide transport in arterioles: frequency- vs. amplitude-dependent control of cGMP formation," Am J Physiol Heart Circ Physiol, vol. 286, pp. H1043-56, Mar 2004.

[25] M. W. Vaughn, et al., "Erythrocytes possess an intrinsic barrier to nitric oxide consumption," J Biol Chem, vol. 275, pp. 2342-8, Jan 282000.

[26] J. F. Leikert, et al., "Reliable in vitro measurement of nitric oxide released from endothelial cells using low concentrations of the fluorescent probe 4,5diaminofluorescein," FEBS Lett, vol. 506, pp. 131-4, Oct 52001.

[27] T. Nagano, "Practical methods for detection of nitric oxide," Luminescence, vol. 14, pp. 283-90, Nov-Dec 1999.

[28] T. Nagano and T. Yoshimura, "Bioimaging of nitric oxide," Chem Rev, vol. 102, pp. 1235-70, Apr 2002.

[29] N. Soh, et al., "A fluorescent probe for monitoring nitric oxide production using a novel detection concept," Analyst, vol. 126, pp. 564-6, May 2001.

[30] M. Chvanov, et. al.,, "Calcium-dependent release of NO from intracellular Snitrosothiols," EMBOJ, vol. 25, pp. 3024-3032, 2006.

[31] S. L. Hempel, et. al., "Dihydrofluorescein diacetate is superior for detecting intracellular oxidants: comparison with 2',7'-dichlorodihydrofluorescein diacetate, 5(and 6)-carboxy-2',7'-dichlorodihydrofluorescein diacetate, and dihydrorhodamine 123," Free Radic Biol Med, vol. 27, pp. 146-159, 1999.

[32] H. Kojima, et al., "Development of a fluorescent indicator for nitric oxide based on the fluorescein chromophore," Chem Pharm Bull (Tokyo), vol. 46, pp. 373-5, Feb 1998.

[33] H. Kojima, et al., "Visualization of oxygen-concentration-dependent production of nitric oxide in rat hippocampal slices during aglycemia," $J$ Neurochem, vol. 76, pp. 1404-10, Mar 2001. 
[34] M. H. Lim and S. J. Lippard, "Fluorescent nitric oxide detection by copper complexes bearing anthracenyl and dansyl fluorophore ligands," Inorg Chem, vol. 45, pp. 8980-9, Oct 302006.

[35] E. Sasaki, et al., "Highly sensitive near-infrared fluorescent probes for nitric oxide and their application to isolated organs," J Am Chem Soc, vol. 127, pp. 3684-5, Mar 232005.

[36] H. Kojima, et al., "Detection and imaging of nitric oxide with novel fluorescent indicators: diaminofluoresceins," Anal Chem, vol. 70, pp. 2446-53, Jul 11998.

[37] X. B. Ji and T. C. Hollocher, "Mechanism for nitrosation of 2,3diaminonaphthalene by Escherichia coli: enzymatic production of NO followed by O2-dependent chemical nitrosation," Appl Environ Microbiol, vol. 54, pp. 1791-4, Jul 1988.

[38] (2001, 06/2006). Nitric Oxide Indicators: DAF-FM and DAF-FM diacetate. Available: http://probes.invitrogen.com/media/pis/mp23841.pdf

[39] A. Balcercyk, et. al.,, "On the specificity of 4-amino-5-methylamino-2',7'difluorofluorescein as a probe for nitric oxide," Free Radic Biol Med, vol. 39, pp. 327-335, 2005.

[40] M. Broillet, et al., "Photoactivation and calcium sensitivity of the fluorescent NO indicator 4,5-diaminofluorescein (DAF-2): implications for cellular NO imaging," FEBS Lett, vol. 491, pp. 227-32, Mar 2001. 


\section{Chapter 2}

KINETIC ANALYSIS OF DAF-FM ACTIVATION BY NO:

TOWARD CALIBRATION OF A NO-SENSITIVE FLUORESCENT DYE

This chapter was submitted as follows (with only slight modifications): Shabnam M. Namin, Sara Nofallah, Mahesh S. Joshi, Konstantinos Kavallieratos, and Nikolaos M. Tsoukias Nitric Oxide (in review). 


\subsection{Abstract:}

Nitric oxide (NO) research in biomedicine has been hampered by the absence of a method that will allow quantitative measurement of NO in biological tissues with high sensitivity and selectivity, and with adequate spatial and temporal resolution. 4-amino-5methylamino-2',7'-difluorofluorescein (DAF-FM) is a NO sensitive fluorescence probe that has been used widely for qualitative assessment of cellular NO production. However, calibration of the fluorescent signal and quantification of NO concentration in cells and tissues using fluorescent probes, have provided significant challenge. In this study we utilize a combination of mathematical modeling and experimentation to elucidate the kinetics of NO/DAF-FM reaction in solution. Modeling and experiments suggest that the slope of fluorescent intensity (FI) can be related to NO concentration according to the equation: $\frac{\mathrm{d}}{\mathrm{dt}}[\mathrm{FI}]=2 \alpha \mathrm{k}_{1}[\mathrm{NO}]^{2}\left[\mathrm{O}_{2}\right] \frac{[\mathrm{DAF}]}{\mathrm{K}_{\mathrm{m}}^{\prime}[\mathrm{NO}]+[\mathrm{DAF}]}$ where $\alpha$ is a proportionality coefficient that relates FI to unit concentration of activated DAF-FM, $\mathrm{k}_{1}$ is the NO oxidation rate constant, and $\mathrm{K}_{\mathrm{m}}^{\prime}$ was estimated to be $4.3 \pm 0.6$. The FI slope exhibits saturation kinetics with DAF-FM concentration. Interestingly, the effective halfmaximum constant increases proportionally to NO concentration. This result is not in agreement with the proposition that $\mathrm{N}_{2} \mathrm{O}_{3}$ is the $\mathrm{NO}$ oxidation byproduct that activates DAF-FM. Kinetic analysis suggests that the reactive intermediate should exhibit NOdependent consumption and thus $\mathrm{NO}_{2}^{\bullet}$ is a more likely candidate. The derived rate law can be used for the calibration of DAF-FM fluorescence and for future quantification of NO concentration in biological tissues. 


\subsection{Introduction}

The measurement of nitric oxide (NO) in biological samples has been hampered by the lack of sensitive probes that can detect NO in nanomolar levels without interference from other species. The available methods for NO detection include the colorimetric Griess reaction, chemiluminescence, electron paramagnetic resonance, electrochemical and fluorometric analyses $[1,2,3]$. Available assays to asses NO availability measure free $\mathrm{NO}$ or $\mathrm{NO}$ oxidation products (i.e. $\mathrm{NO}_{2}^{-}, \mathrm{NO}_{3}^{-}, \mathrm{N}_{2} \mathrm{O}_{3}$ ) and signaling derivatives (i.e. S-nitrosothiols, cGMP). Available methods vary in their sensitivity, specificity and interference from biological constituents. NO is highly reactive with other modalities and has a very short half-life and steep concentration gradients. Determining local availability and intracellular concentrations has proven to be quite challenging.

Fluorometric methods have been used to assess intracellular NO levels [4]. The development of fluorescent probes in recent years has provided sensitive and specific assays that are gaining wide applications in measuring nitrogen oxides $[5,6,7,8,9,10$, 11]. NO-reactive fluorescent indicators allow bio-imaging of NO with high spatial resolution, permitting investigation of two-dimensional NO production in real time by digital fluorescence imaging, which is not possible with other methods [7]. These fluorescent probes may be used to measure nanomolar levels of NO in vitro and in vivo [12]. Diamine derivatives of fluorescein are the most widely used NO fluorescent probes (i.e. 4,5 Diaminofluorescein (DAF-2) and 4-amino-5-methylamino-2',7'difluorofluorescein (DAF-FM)). Exposure of these compounds to NO, generates highly 
fluorescent triazole derivatives (DAF-T) [13]. Fluorescence to NO, however, is achieved only under aerobic conditions, indicating that DAF reacts with an oxidative product of NO, rather than NO itself [14]. The nature of the NO active derivative has not been established, although $\mathrm{N}_{2} \mathrm{O}_{3}$ has been proposed as a candidate for the reactive intermediate [14]. An alternative reaction scheme has also been proposed [15] where activation of DAF occurs through a two step process by initially reacting with $\mathrm{NO}_{2}$ radical. Therefore, controversy exists as to the actual kinetic mechanism for the reaction between DAF and NO.

The vast majority of studies incorporating the use of DAFs have only reported relative changes in fluorescent signal $[16,17,18,19,20]$. Quantification of NO concentration ([NO]) in biological tissues using fluorescent probes is hindered by a series of challenges, including uneven dye loading, dye leakage, motion artifacts, photoactivation and photobleaching [14, 21, 22]. In addition, the dye may also exhibit increase in fluorescence due to the presence of cations [23]. Most importantly, elucidating the kinetics of NO-dye interaction is a prerequisite for developing meaningful calibration protocols. In this study, we utilize mathematical modeling and experimentation to investigate the reaction of NO with DAF-FM and to gain further insight into the actual intermediate that activates DAFs. Based on the proposed mechanism and kinetic law, recommendations for calibration of DAF fluorescence and the quantification of NO in biological tissues are provided. 


\subsection{Methods}

Kinetic analysis. We investigated the kinetic mechanism that leads to activation of DAF by NO and the formation of the triazole derivative, DAF-T. Assuming that the intermediate product of NO's autoxidation reacting with DAF is $\mathrm{N}_{2} \mathrm{O}_{3}$, [24] DAF-T formation will proceed through the following reactions [7, 20, 25].

$$
\begin{aligned}
& 2 \mathrm{NO}+\mathrm{O}_{2} \underset{\mathrm{k}_{-2}}{\stackrel{\mathrm{k}_{1}}{\longrightarrow}} 2 \mathrm{NO}_{2}^{\cdot} \\
& \mathrm{NO}_{2}^{\cdot}+\mathrm{NO}_{3} \stackrel{\mathrm{k}_{2}}{\longrightarrow} \mathrm{O}_{3} \\
& \mathrm{~N}_{2} \mathrm{O}_{3}+\mathrm{H}_{2} \mathrm{O} \stackrel{\mathrm{k}_{3}}{\longrightarrow} 2 \mathrm{NO}_{2}^{\cdot}+2 \mathrm{H}^{+} \\
& \mathrm{N}_{2} \mathrm{O}_{3}+\mathrm{DAF} \stackrel{\mathrm{k}_{4}}{\longrightarrow} \mathrm{DAF}-\mathrm{T}
\end{aligned}
$$

Reaction rate constants $\mathrm{k}_{1}\left(3.15 \times 10^{6} \mathrm{M}^{-2} \mathrm{~s}^{-1}\right), \mathrm{k}_{2}\left(1.1 \times 10^{9} \mathrm{M}^{-1} \mathrm{~s}^{-1}\right), \mathrm{k}_{-2}\left(8.1 \times 10^{4} \mathrm{~s}^{-1}\right)$, and $\mathrm{k}_{3}$ $\left(38130 \mathrm{~s}^{-1}\right)$ have been previously reported [26]. Applying the law of mass action to this reaction scheme yields differential equations that can be solved numerically to describe the rates of change of $\mathrm{NO}, \mathrm{NO}_{2}^{*}, \mathrm{~N}_{2} \mathrm{O}_{3}$, and DAF-T (see Appendix 2.1).

Activation of DAF by an oxidation product of NO, rather than NO itself, will affect the functional dependence of the rate of DAF-T formation on the reactants. Assuming that the concentrations of unstable intermediates (i.e. $\mathrm{N}_{2} \mathrm{O}_{3}$ and $\mathrm{NO}_{2}^{*}$ ) are small relative to the reactants, and therefore show negligible rates of change, a Pseudo Steady State Approximation (PSSA) can be employed for their concentrations. This simplifies the proposed kinetic mechanism (Reactions 2.1-2.4) and yields Equation 2.1:

$$
\frac{\mathrm{d}}{\mathrm{dt}}[\mathrm{DAF}-\mathrm{T}]=2 \mathrm{k}_{1}[\mathrm{NO}]^{2}\left[\mathrm{O}_{2}\right] \frac{[\mathrm{DAF}]}{\mathrm{k}_{3} / \mathrm{k}_{4}+[\mathrm{DAF}]}
$$


$\gamma=\frac{[D A F]}{\mathrm{k}_{3} / \mathrm{k}_{4}+[\mathrm{DAF}]}$ is the fraction of $\mathrm{N}_{2} \mathrm{O}_{3}$ that is utilized to activate DAF-FM at any given moment (i.e. rate of reaction 2.4 over reaction 2.3 and 2.4). Note that the kinetic mechanism and the simplified kinetic behavior depicted in Equation 2.1 are analogous to the kinetics of nitrosation of thiols by NO in [27]. Equation 2.1 simply states that the formation rate of the triazole derivative, DAF-T, will be limited by the rate of NO oxidation (Reaction 2.1) and the fraction of $\mathrm{N}_{2} \mathrm{O}_{3}$ that reacts with DAF-FM. This approximation was validated against the numerical solution of the system for the assumed parameter values for $\mathrm{k}_{1}, \mathrm{k}_{2}, \mathrm{k}_{-2}, \mathrm{k}_{3}$ and a wide range of values for $\mathrm{k}_{4}$. Assuming a constant fraction $\gamma$, Equation 2.1 can be integrated to give the increase in DAF-T after the addition of a bolus amount of NO. For limiting initial NO concentrations $\left([\mathrm{NO}]_{\mathrm{i}}\right)$ this increase will be approximately equal to:

$$
\Delta[\mathrm{DAF}-\mathrm{T}]=\gamma / 2[\mathrm{NO}]_{\mathrm{i}}
$$

Thus, a linear dependence between fluorescent intensity (FI), and total amount of NO added, is possible under some conditions despite a square dependence of the increase in FI on $[\mathrm{NO}]$ at any particular instance.

Materials. Dulbecco's phosphate buffered saline was purchased from Invitrogen (Grand Island, NY). DAF-FM was from Molecular Probes (Eugene, OR). Spermine NONOate (SPER/NO) was from Cayman Chemicals (Ann Arbor, MI). DMSO (dimethyl sulfoxide anhydrous) was purchased from Sigma-Aldrich (St. Louis, MO), and the sodium hydroxide $(\mathrm{NaOH}), 2 \mathrm{~N}$ solution was from Fisher Scientific (Fair Lawn, NJ). A 7 
mM stock solution of DAF-FM was made with DMSO. Dilutions to final working concentrations were done by the addition of DPBS. Spermine NONOate $\left(\mathrm{t}_{1 / 2}=230\right.$ minutes at $22-25^{\circ} \mathrm{C}$ ) was chosen as a slow releasing $\mathrm{NO}$ donor. Final working concentrations were made with DPBS immediately prior to experimentation.

Measurement of NO. We investigated the NO released from a NO donor (SPER/NO) at $25^{\circ} \mathrm{C}$, obtaining the resulting [NO] profile using a $\mathrm{NO}$ sensitive electrode (ISONOP, WPI; Sarasota, FL). Different concentrations of the NO donor (50 nM to 500 $\mu \mathrm{M})$, were prepared in an aerated buffered solution ( $\mathrm{pH}$ 7.4). The NO released was detected using the electrode. The reaction of DAF-FM with NO liberated from the NO donor was monitored using a computer controlled automatic microplate fluorometer (GENios; TECAN, Inc.; Durham, NC). Samples were loaded in black 96-well plates and fluorescence intensity (FI) was recorded at an emission wavelength $535 \mathrm{~nm}$ followed excitation at $485 \mathrm{~nm}$. FI increase with time was recorded at different combinations of DAF-FM and NO donor concentrations to assess the dependency of FI on both DAF-FM and NO.

\subsection{Results}

The "Clamped NO" protocol. First, we examined the NO release pattern from SPER/NO in aerated solution. An NO donor, with a relatively long half-life, can provide continuous release of $\mathrm{NO}$ over the duration of a typical experiment ( 15 minutes). Figure 2.1A shows representative model simulations (using the model in Appendix 2.1) for the evolution of $\mathrm{NO}$ in an aerated solution $\left(\left[\mathrm{O}_{2}\right]=2.77 \times 10^{-4} \mathrm{M}\right)$ at three different SPER/NO concentrations $(10,500$ and $1000 \mu \mathrm{M})$. NO is released and accumulates until reaching a maximum concentration (represented by a circle) that is followed by a slowly 
decaying plateau. The time it takes to reach this plateau differs depending on the concentration of the NO donor and its assumed half-life. At higher concentrations, this plateau is established faster. This offers a relative steady level of NO which can be maintained over the duration of an experiment, similar to the "clamped NO protocol" in [28]. (Note that the "clamped NO protocol" of this earlier study utilizes CPTIO rather than dissolved $\mathrm{O}_{2}$ as the main route of $\mathrm{NO}$ consumption.)
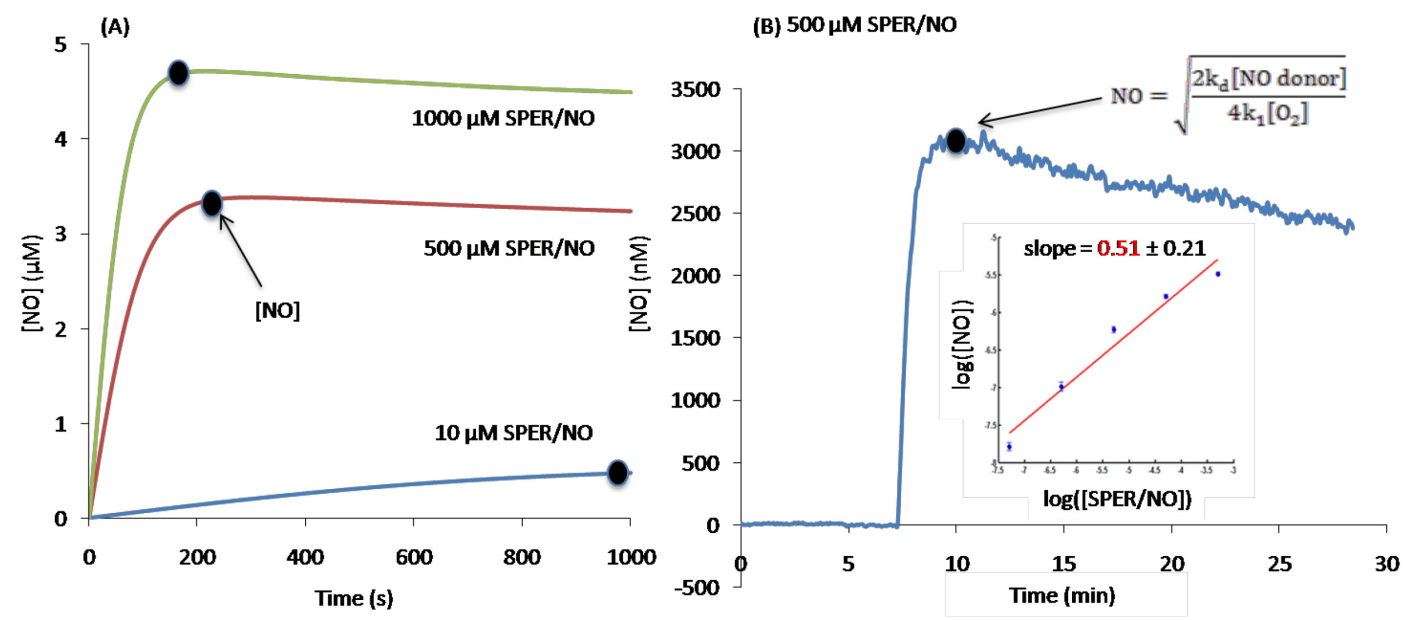

Figure 2.1 - (A) Predicted continuous release by 1000, 500 and $10 \mu \mathrm{M}$ SPER/NO. Maximum NO release is indicated by the dot with a constant increase until a pseudo steady-state plateau is reached. (B) NO release from $500 \mu \mathrm{M}$ SPER/NO over time, detected by an $\mathrm{NO}$ electrode $(\mathrm{n}=4)$. The dot indicates when maximum [NO] achieved. (B-Insert) Log-log plot displays the relationship between [NO] and [SPER/NO] using maximum [NO] from ranging [SPER/NO] detected by the electrode. The linear fit of the $\log -\log$ plot has a slope of 0.508 with a standard deviation of 0.208 . The slope is not significantly different than $0.5(\mathrm{p}=0.95)$.

This relative steady level of NO, during the plateau phase, is a result of the balance between $\mathrm{NO}$ release by the NO donor (S) and NO consumed by the rate limiting oxidation reaction of $\mathrm{NO}\left(\mathrm{R}=\sim 4 \mathrm{k}_{1}[\mathrm{NO}]^{2}\left[\mathrm{O}_{2}\right]\right)$, and can be approximated by Equation 2.3: 


$$
[\mathrm{NO}] \approx \sqrt{\frac{\mathrm{S}}{4 \mathrm{k}_{1}\left[\mathrm{O}_{2}\right]}}=\sqrt{\frac{\mathrm{nk}_{\mathrm{d}}[\mathrm{NOdonor}]}{4 \mathrm{k}_{1}\left[\mathrm{O}_{2}\right]}}
$$

where $k_{d}$ is the dissociation rate constant $\left(k_{d}=\ln 2 / t_{1 / 2}\right)$, and $n$ is the moles of NO released for every mole of $\mathrm{NO}$ donor. In Equation 2.3 we have assumed a constant release rate for the NO donor which is a valid assumption for $t_{\text {experiment }}<\frac{1}{10} t_{1 / 2}$. Thus, a 100 times increase in [NO donor] (i.e. 10 and $1000 \mu \mathrm{M}$ SPER/NO) should yield only a 10-fold increase in "clamped" [NO], given the square relationship.

The theoretical prediction for a square dependence between NO and NO donor concentration was verified experimentally. Figure $2.1 \mathrm{~B}$ shows a representative tracing using the NO-sensitive electrode. NO evolves in a buffered solution of $500 \mu \mathrm{M}$ SPER/NO. After a short accumulation phase, a maximum [NO] is reached. A slight decay after the peak is observed as the NO donor is slowly consumed over time. The maximum NO concentration reached was recorded and average values $(n=4)$ at different SPER/NO concentrations (50 nM to $500 \mu \mathrm{M})$ are summarized in a log-log plot (Insert in Figure 2.1B). A linear fit of the data yields a slope of $0.51 \pm 0.21$. The slope is not significantly different than $0.5(\mathrm{p}=0.95)$. Thus, experimental data is in agreement with the theoretical predictions suggesting that the $[\mathrm{SPER} / \mathrm{NO}]$ is proportional to $[\mathrm{NO}]^{2}$.

Dependence of fluorescence intensity on DAF and NO concentrations. A representative numerical simulation from the model in the Appendix 2.1 is presented in Figure 2.2A. DAF-T formation is depicted as a function of time, for $5 \mu \mathrm{M}$ of DAF-FM and $50 \mu \mathrm{M}$ of SPER/NO. At $\mathrm{t}=0$, the slope of the curve is zero as a result of the absence 
of free NO initially. DAF-T formation begins to increase as NO evolves, until a maximum rate of change is reached (i.e. maximum slope, $\beta_{\max }$ ). This corresponds to the point of maximum NO concentration in Figures 2.1A and 2.1B. Thus, the $\beta_{\max }$ of the DAF-T formation curve, can be correlated to the clamped NO concentration levels.

Assuming that DAF-T is the main fluorescently active species in the solution, we expect that FI will be proportional to the DAF-T concentration. Thus, FI should exhibit a similar profile in time with the [DAF-T] generated from the model. Figure 2.2B depicts a representative experimental tracing of FI upon mixing of [DAF-FM] and [SPER/NO] (5 $\mu \mathrm{M}$ and $50 \mu \mathrm{M}$ final concentrations respectively) in the fluorometer. Experimental tracing of FI and model predictions for [DAF-T] are in agreement.
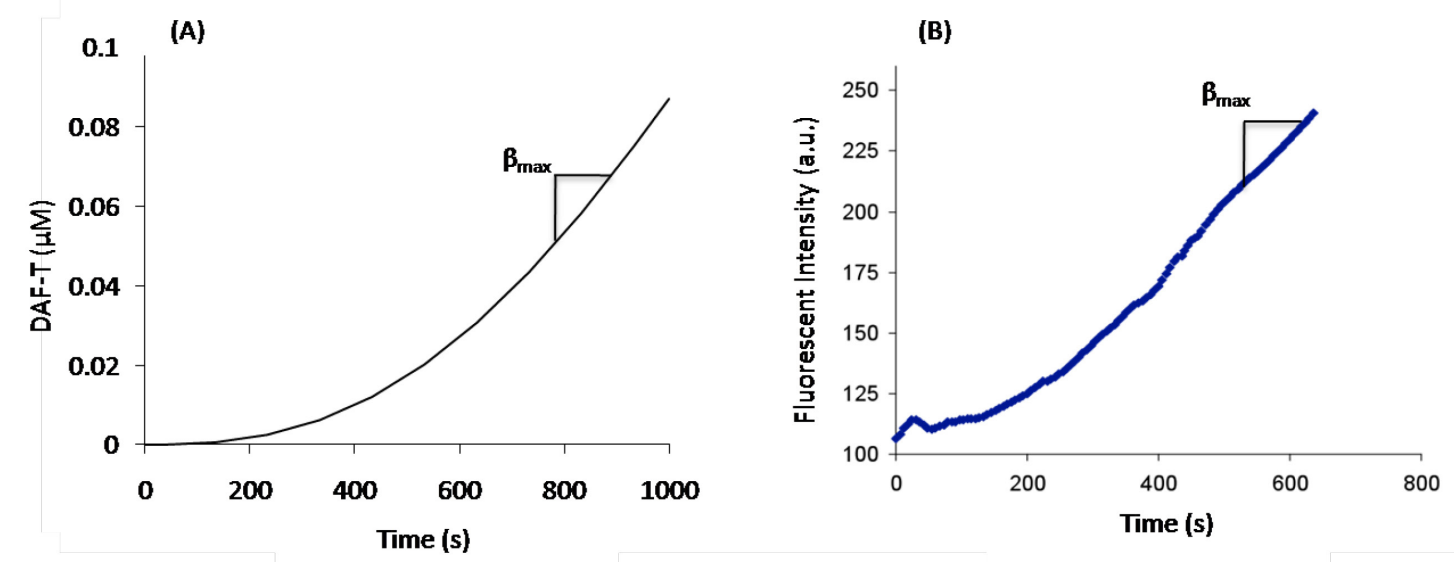

Figure 2.2 - Fluorescent intensity curves obtained from solutions containing $5 \mu \mathrm{M}$ DAFFM and $50 \mu \mathrm{M}$ SPER/NO (A) simulation (B) experimental, indicating when $\beta_{\max }$ is achieved.

Fluorescent intensity vs. NO donor. In a series of experiments, we investigated the dependence of FI on [NO] by varying the concentration of SPER/NO, while keeping the concentration of DAF-FM constant at $5 \mu \mathrm{M}$. Fluorescence measurements were taken 
in the fluorometer and representative results are depicted in Figure 2.3A for SPER/NO concentrations of $10 \mu \mathrm{M}, 100 \mu \mathrm{M}$ and $1 \mathrm{mM}$. As expected, we observed an increase in maximum slope $\left(\beta_{\max }\right)$ when increasing $[\mathrm{SPER} / \mathrm{NO}]$. To test the relationship between NO donor and $\beta_{\max }$, average maximum slopes $(\mathrm{n}=5)$ were calculated for six different SPER/NO concentrations $(10-1000 \mu \mathrm{M})$. Data is summarized in Figure 2.3B as a loglog plot. A linear fit gives a slope of $0.99 \pm 0.03$. This slope is not significantly different than $1(\mathrm{p}=0.74)$. Therefore, experiments show a maximum slope in FI $\left(\beta_{\max }\right)$ that is proportional to NO donor concentration. Data in Figures 2.1-2.3 combined, suggests that $\beta_{\max }$ is also proportional to $[\mathrm{NO}]^{2}$. This is in agreement with the kinetic analysis (Equation 2.1).
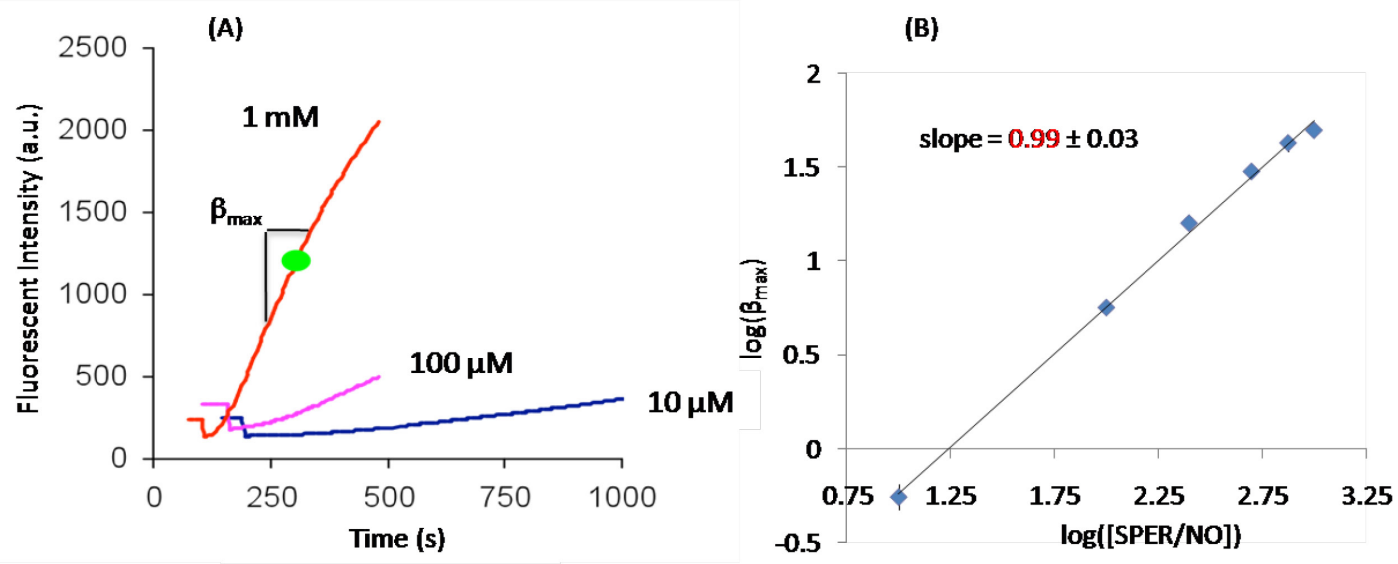

Figure 2.3 - (A) Fluorescent intensity curves showing the dependence of [SPER/NO] (10 $\mu \mathrm{M}, 100 \mu \mathrm{M}$, and $1 \mathrm{mM}$ ) on slope $\left(\beta_{\max }\right)$. (B) Log-log plot of $\beta_{\max }$ and [SPER/NO] $(\mathrm{n}=5)$. A linear relationship is observed having a slope of 0.99 with a standard deviation of 0.03 . This slope is not significantly different than $1(\mathrm{p}=0.74)$.

Fluorescent intensity vs. DAF-FM. To investigate the dependence of the FI on DAF, a series of experiments were performed by varying [DAF-FM] at constant [SPER/NO]. Experiments were repeated for different [SPER/NO]. For each combination of [DAF-FM] and [SPER/NO], the maximum slope $\beta_{\max }$ of the FI curve was 
recorded. Figure 2.4A depicts representative results. Average $\beta_{\max }$ values $(n=5)$ are presented as a function of DAF-FM concentration and for two different NO donor concentration. We observe saturation dependence of $\beta_{\max }$ on DAF-FM and thus a Michaelis-Menten equation was utilized to fit the data.

$$
\beta_{\text {max }}=\max \beta_{\text {max }} \frac{[\mathrm{DAF}]}{\mathrm{K}_{\mathrm{m}}+[\mathrm{DAF}]}
$$

where $\max \beta_{\max }$ is the maximum $\beta_{\max }$ at saturating DAF concentrations, and $\mathrm{K}_{\mathrm{m}}$ is the concentration of DAF for half-maximum $\beta_{\max }$.

Fitting with Equation 2.4 allows us to estimate $\max \beta_{\max }$ and $\mathrm{K}_{\mathrm{m}}$ at each [SPER/NO]. $\max \beta_{\max }$ increases linearly with SPER/NO concentrations (i.e. $\max \beta_{\max }$ is 20.1 and 1.9 for $125 \mu \mathrm{M}$ and $12.5 \mu \mathrm{M}$ of SPER/NO respectively). This linear relationship has been previously noted $[20,29]$. Therefore, the slope $\beta_{\max }$ of the FI curve shows a linear dependence on $[\mathrm{SPER} / \mathrm{NO}]$ and thus exhibits a square dependence on [NO]. This is in agreement with Equation $2.1\left(\max \beta_{\max }=2 \mathrm{k}_{1}[\mathrm{NO}]^{2}\left[\mathrm{O}_{2}\right]\right)$. Surprisingly, however, the estimated $\mathrm{K}_{\mathrm{m}}$ values also increase with $\mathrm{NO}$ donor concentrations (i.e. $\mathrm{K}_{\mathrm{m}}$ is 5.3 and 1.0 for $125 \mu \mathrm{M}$ and $12.5 \mu \mathrm{M}$ of SPER/NO respectively). This was not anticipated based on Equation 2.1 and thus, the observed dependence of $\mathrm{K}_{\mathrm{m}}$ on $\mathrm{NO}$ questions the proposed kinetic mechanism of DAF-FM activation by $\mathrm{N}_{2} \mathrm{O}_{3}$ (Reactions 2.1-2.4) 

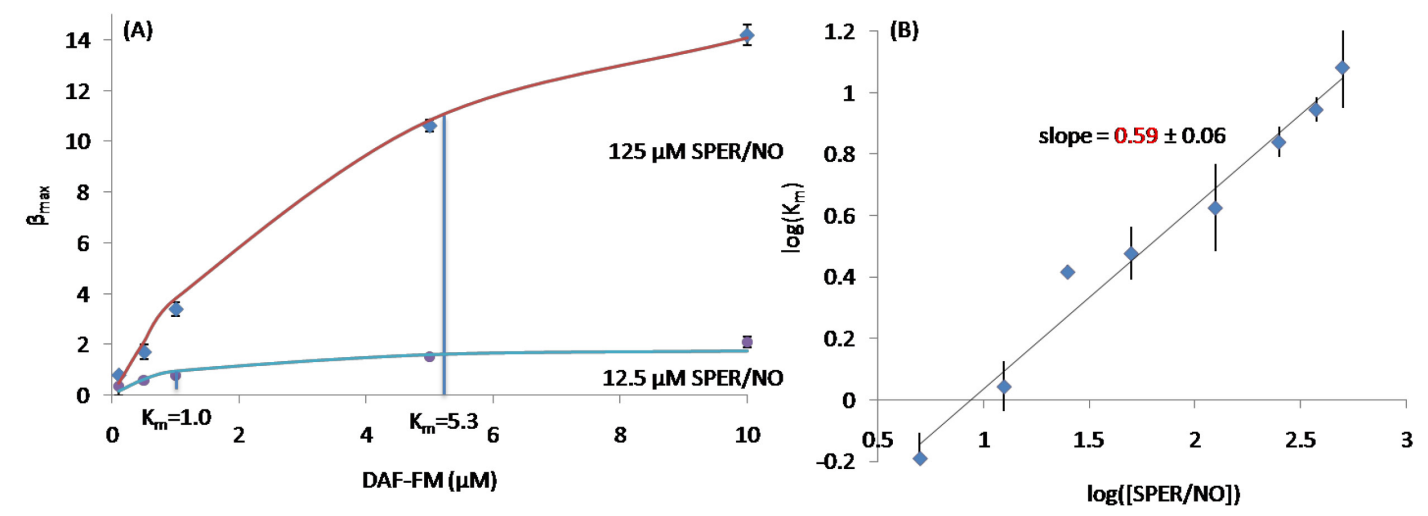

Figure 2.4 - (A) Data fitting for $\beta_{\max }$ with respect to [DAF-FM] at 12.5 and $125 \mu \mathrm{M}$ [SPER/NO] $(n=5)$ indicating $K_{m}$ values of 1.0 and 5.3 respectively. (B) Log-log plot of $\mathrm{K}_{\mathrm{m}}$ and [SPER/NO] $(\mathrm{n}=5)$ with a linear relationship having a slope of 0.59 and a standard deviation of 0.06 . The slope of this line is statistically different than $1\left(p<5.5 \times 10^{-5}\right)$ and 0 $\left(\mathrm{p}<1.2 \times 10^{-5}\right)$ and close to 0.5 .

Experiments at eight different SPER/NO concentrations $(5-500 \mu \mathrm{M})$ were performed. At each NO donor concentration, five different DAF-FM concentrations $(0.1$ $10 \mu \mathrm{M})$ were utilized to estimate a single $\mathrm{K}_{\mathrm{m}}$ value for each combination. The log-log plot of the average $K_{m}$ values from $n=5$ repetitions are depicted in Figure 2.4B. A linear fit of this line has a slope of $0.59 \pm 0.06$. The slope of this line is statistically different than $1\left(\mathrm{p}<5.5 \times 10^{-5}\right)$ and $0\left(\mathrm{p}<1.2 \times 10^{-5}\right)$ and close to 0.5 . Thus, our data points toward a $\mathrm{K}_{\mathrm{m}}$ that is proportional to [SPER/NO] ${ }^{1 / 2}$ and thus proportional to [NO]. 


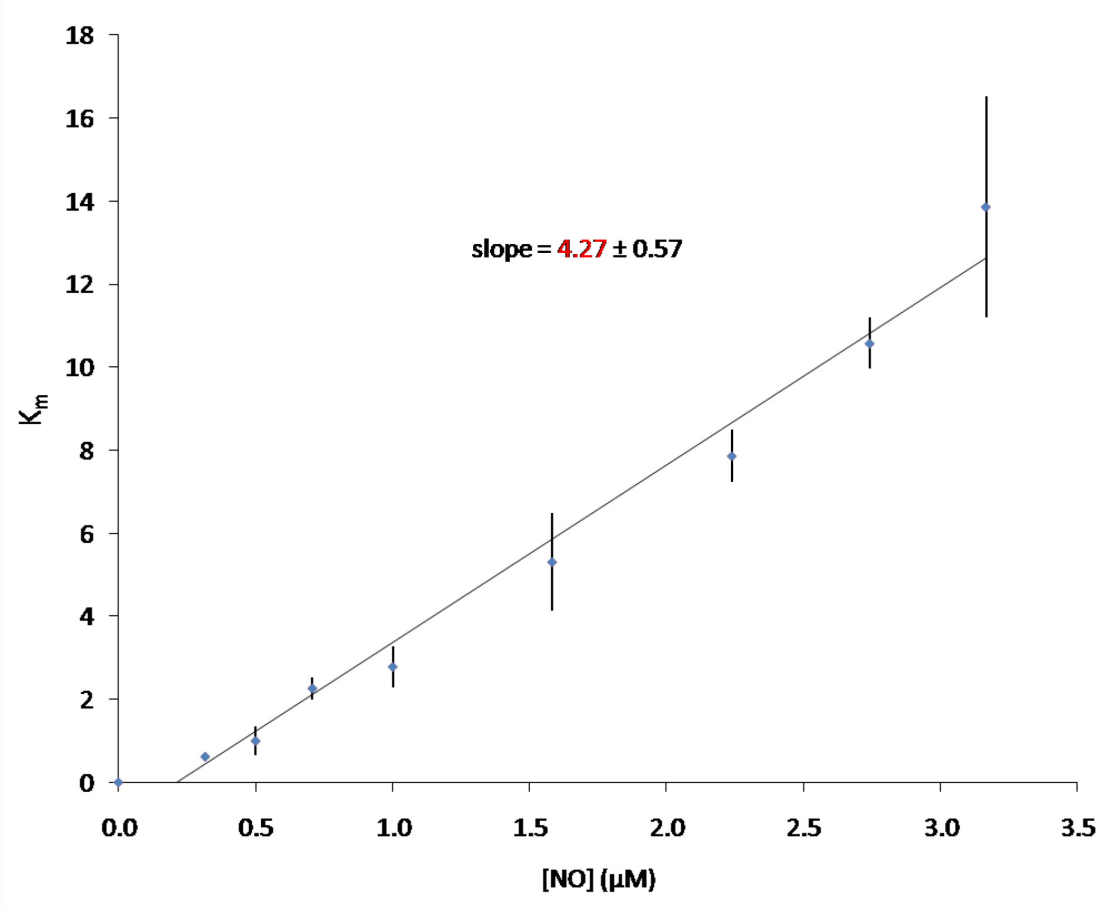

Figure 2.5 - Relationship of the dependence of $\mathrm{K}_{\mathrm{m}}$ on [NO]. The linear slope of the line yields the value of $K_{m}^{\prime}=4.27$ with a standard deviation of 0.57 .

Figure 2.5 shows the dependence of $\mathrm{K}_{\mathrm{m}}$ on NO. SPER/NO concentrations were translated to NO concentrations based on Equation 2.3 and NO-electrode recordings that show $500 \mu \mathrm{M}$ NO donor correspond to $3.2 \mu \mathrm{M}$ NO. A linear fit of the data in Figure 2.5 gives:

$$
\mathrm{K}_{\mathrm{m}}=\mathrm{K}_{\mathrm{m}}^{\prime}[\mathrm{NO}]
$$

with $\mathrm{K}_{\mathrm{m}}^{\prime}=4.3 \pm 0.6$. Equations 2.1, 2.4 and 2.5 suggest the following rate law for DAF$\mathrm{T}$ formation rate.

$$
\frac{\mathrm{d}}{\mathrm{dt}}[\mathrm{DAF}-\mathrm{T}]=2 \mathrm{k}_{1}[\mathrm{NO}]^{2}\left[\mathrm{O}_{2}\right] \frac{[\mathrm{DAF}]}{\mathrm{K}_{\mathrm{m}}^{\prime}[\mathrm{NO}]+[\mathrm{DAF}]}
$$


Revised Kinetic Mechanism. Equation 2.6 suggests that the fraction of the unknown NO byproduct $\mathrm{X}$ that reacts with DAF-FM is $\gamma^{\prime}=\frac{[\mathrm{DAF}]}{\mathrm{K}_{\mathrm{m}}^{\prime}[\mathrm{NO}]+[\mathrm{DAF}]}$. An effective $\mathrm{K}_{\mathrm{m}}$ that is $\mathrm{NO}$ dependent can be achieved if this active intermediate is consumed through an NO-dependent pathway. (i.e. $\gamma^{\prime}$ is the rate of reaction of $\mathrm{X}$ with DAF over the rate of reaction with DAF plus the rate of NO-dependent consumption of $\mathrm{X})$.

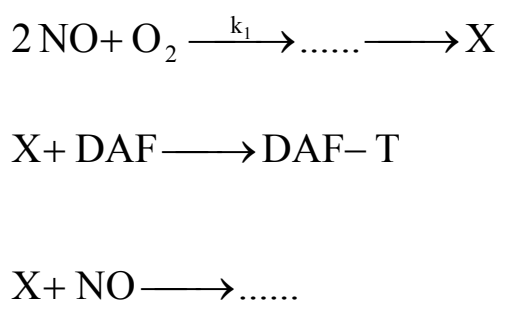

Thus, our data argues against the proposition that this species is $\mathrm{N}_{2} \mathrm{O}_{3}$. Previous studies have argued for a different reaction mechanism where conversion of DAF to DAF-T occurs after DAF is initially oxidized $[15,22,30] . \mathrm{NO}_{2}^{\bullet}$ is proposed to be this oxidating agent, and the measurement of the fluorescently derived triazole product reflects this oxidation. $\mathrm{N}_{2} \mathrm{O}_{3}$ has also been suggested as the active intermediate involved in thiol nitrosation [27]. However, recent work has provided evidence for a more important role of $\mathrm{NO}_{2}^{\cdot}$, rather than $\mathrm{N}_{2} \mathrm{O}_{3}$, in thiol nitrosation $[31,32,33,34]$. The resemblance of the kinetic behavior between DAF activation and GSH nitrosation, also makes $\mathrm{NO}_{2}^{*}$ an attractive candidate for the unidentified reactive intermediate in DAF activation by NO. A potential kinetic mechanism that assumes $\mathrm{NO}_{2}^{*}$ as the oxidative product of $\mathrm{NO}$ that 
reacts with DAF would include Reaction 2.1-2.3 as well as Reaction 2.5 instead of Reaction 2.4.

$$
\mathrm{NO}_{2}^{\bullet}+\mathrm{DAF} \stackrel{\mathrm{k}_{5}}{\longrightarrow} \ldots \ldots \longrightarrow \mathrm{DAF}-\mathrm{T}
$$

Assuming the DAF oxidation by $\mathrm{NO}_{2}^{-}$is the rate limiting step in Reaction 2.5, a PSSA can be utilized to simplify Reactions $2.1,2.2,2.3$, and 2.5 yielding the rate of DAF-T formation:

$$
\frac{\mathrm{d}}{\mathrm{dt}}[\mathrm{DAF}-\mathrm{T}]=2 \mathrm{k}_{1}[\mathrm{NO}]^{2}[\mathrm{O}]_{2} \frac{[\mathrm{DAF}]}{\left(\frac{\mathrm{k}_{3} \mathrm{k}_{2}}{\mathrm{k}_{5}\left(\mathrm{k}_{-2}+\mathrm{k}_{3}\right)}\right)[\mathrm{NO}]+[\mathrm{DAF}]} \text { (Equation 2.7) }
$$

Comparing Equation 2.7 with Equation 2.6 we get $\mathrm{K}_{\mathrm{m}}^{\prime}=\left(\frac{\mathrm{k}_{3} \mathrm{k}_{3}}{\mathrm{k}_{5}\left(\mathrm{k}_{-2}+\mathrm{k}_{3}\right)}\right)=4.3 \pm 0.6$.

Based on the values for the other reaction rate constants, we can an estimate a value for $\mathrm{k}_{5}$ in the reaction of DAF-FM activation to be $8.2 \times 10^{7} \mathrm{M}^{-1} \mathrm{~s}^{-1}$. This value is of the same order as the oxidative rate constant of similar vicinal diamines by $\mathrm{NO}_{2}^{\bullet}$ (i.e. rate constant for 1,4-phenylenediamine is $\left.4.6 \times 10^{7} \mathrm{M}^{-1} \mathrm{~s}^{-1}[30,35]\right)$.

\subsection{Discussion}

A number of methods have been employed to measure NO and related nitrogen oxides. NO sensitive fluorescent probes have often been used to detect NO levels, however, most studies provide data in relative fluorescence and quantification of NO concentration fluorescently has not been accomplished. This is due to several experimental limitations that may arise when utilizing DAF in many systems (i.e. motion 
artifacts, uneven dye loading, photosensitivity and dye leakage) as well as due to an incomplete understanding of the kinetics of DAF-NO reaction. Characterizing the active intermediate for DAF activation by analyzing the kinetic mechanism between DAF and NO will aid in overcoming some of these limitations.

Active intermediate of DAF activation. The dependence of DAF fluorescence on the presence of $\mathrm{O}_{2}$, suggests that DAF is activated by a byproduct of $\mathrm{NO}$ oxidation rather than $\mathrm{NO}$ itself. $\mathrm{N}_{2} \mathrm{O}_{3}$ has been previously proposed as an intermediate that can play this role. An alternative reaction mechanism has also been proposed according to which the activation of DAF occurs as a two-step process [30]. The first step is the oxidation of DAF via a one electron species such, as $\mathrm{NO}_{2}^{\circ}$, yielding a nitrosamine intermediate that reacts further with NO to form the fluorescent triazole DAF-T. The data presented in this study supports the second mechanism of $\mathrm{NO}_{2}^{*}$ as the reactive intermediate in DAF activation $[15,22,30]$ and suggests the initial oxidation step to be rate-limiting in this two-step conversion process.

A mechanism based on $\mathrm{NO}_{2}^{*}$ rather than $\mathrm{N}_{2} \mathrm{O}_{3}$ as the reactive intermediate would exhibit similar, but not identical kinetic behavior (Equation 2.1 vs. Equation 2.7). In both mechanisms, DAF-T formation is limited by the rate of NO oxidation and both exhibit Michaelis-Menten dependence on DAF concentration. However, based on the second mechanism, the effective $K_{m}$ is NO dependent. Experimental data (Figure 2.4) demonstrates such a dependence of $\mathrm{K}_{\mathrm{m}}$ on $[\mathrm{NO}]$ and point towards $\mathrm{NO}_{2}^{*}$ as the active intermediate for DAF activation. However, any NO oxidation byproduct, that would exhibit NO dependent consumption, could satisfy the observed behavior. 
Use of NO Donors for calibration standards. Theoretical considerations, corroborated by experiments, suggest that slow releasing NO donors can provide steady levels of NO concentrations in aerated buffered solutions. This is ideally suited for creating calibration standards. The half-life of the NO donor needs to be significant relative to the duration of the experiment (i.e. $t_{1 / 2}>10 \cdot t_{\text {experiment }}$ ) such as no significant consumption of NO donor occurs over this time frame. The "clamped NO" concentration will depend not only on the stoichiometry, half-life, and concentration of NO donor, but also on the rate of NO consumption. In the absence of other NO scavengers, the reaction with dissolved $\mathrm{O}_{2}$ will limit this rate and will lead to a square dependence between [NO donor] and [NO]. An appropriate time from the initiation of $\mathrm{NO}$ donor release is required for reaching this plateau NO concentration. This time can be approximated by the ratio of the NO plateau over the initial release rate (i.e. $\mathrm{t}_{\mathrm{lag}}>\left(4 \mathrm{k}_{1}\left[\mathrm{O}_{2}\right] \mathrm{nk}_{\mathrm{d}}[\mathrm{NOdonor}]\right)^{1 / 2}$. NO electrodes can validate these predictions and test the relationship between [NO] and [NO donor] in a particular system.

Calibration using bolus addition of NO. Studies have sometimes utilized the addition of a solution containing a known NO concentration for assessing NO fluorescence or a fast releasing NO donor [36]. Such an addition of a bolus amount of NO will result in a change in NO fluorescence that will stop when NO (or DAF) is consumed. This information is difficult to interpret as the $[\mathrm{NO}]$ and/or [DAF] concentrations may change significantly during the course of the experiment. Thus, a linear increase in final fluorescence after addition of solutions with different NO concentration does not guarantee a linear dependence of fluorescence on [NO] at any 
particular instance. For example, Equation 2.2 shows that under some conditions the final change in NO fluorescence will be linearly dependent on the amount of NO added on the system, even though at any particular moment the increase in FI is proportional to $[\mathrm{NO}]^{2}$ (Equation 2.6). Thus, a calibration curve using solutions with different NO concentration standards may not be ideal for time-dependent monitoring of NO.

Kinetics of DAF activation. Unlike many other fluorescence indicators that bind reversibly with the molecule of interest, DAF reacts with the active NO derivative in an irreversible manner. The irreversibility of DAF activation has two main implications. First, the fluorescence signal should remain constant and should not decrease when NO disappears. As a result, any observed drop in FI is an artifact that should be attributed to experimental limitations such as dye leakage, motion artifacts, photobleaching etc. Second, the NO concentration levels correlate with the rate of DAF-T formation and thus with the rate of FI increase (i.e. assuming a linear dependence of FI on [DAF-T]). Thus, a relationship between the slope of FI and [NO] (and not NO release rate) should be sought. This relationship will be governed by the reaction mechanism of DAF with NO and based on our theoretical and experimental data can be approximated by:

$$
\frac{\mathrm{d}}{\mathrm{dt}}[\mathrm{FI}]=\alpha \frac{\mathrm{d}}{\mathrm{dt}}[\mathrm{DAF}-\mathrm{T}]=2 \alpha \mathrm{k}_{1}[\mathrm{NO}]^{2}\left[\mathrm{O}_{2}\right] \frac{[\mathrm{DAF}]}{\mathrm{K}_{\mathrm{m}}^{\prime}[\mathrm{NO}]+[\mathrm{DAF}]}(\text { Equation 2.8) }
$$

where $\alpha$ is a proportionality coefficient that depends on the fluorescent yield per mole of DAF-T, and $\mathrm{K}_{\mathrm{m}}^{\prime}$ is estimated from our experiments to be $4.3 \pm 0.6 \mu \mathrm{M} \mathrm{DAF} / \mu \mathrm{M}$ NO.

Inspection of Equation 2.8 suggests two limiting cases. In Case I, when $[\mathrm{DAF}]<<\mathrm{K}_{\mathrm{m}}^{\prime}$ [NO], Equation 2.8 reduces to: 


$$
\frac{\mathrm{d}}{\mathrm{dt}}[\mathrm{FI}]=\alpha \frac{\mathrm{d}}{\mathrm{dt}}[\mathrm{DAF}-\mathrm{T}]=2 \alpha \frac{\mathrm{k}_{1}}{\mathrm{~K}_{\mathrm{m}}^{\prime}}[\mathrm{NO}]\left[\mathrm{O}_{2}\right][\mathrm{DAF}]
$$

This would suggest a DAF-T formation rate that is proportional to [NO] and [DAF] under these conditions.

In case II, when $[\mathrm{DAF}]>>\mathrm{K}_{\mathrm{m}}^{\prime}[\mathrm{NO}]$, Equation 2.8 reduces to:

$$
\frac{\mathrm{d}}{\mathrm{dt}}[\mathrm{FI}]=\alpha \frac{\mathrm{d}}{\mathrm{dt}}[\mathrm{DAF}-\mathrm{T}]=2 \alpha \alpha_{1}[\mathrm{NO}]^{2}\left[\mathrm{O}_{2}\right]
$$

This would yield a DAF-T formation that is independent of the [DAF] and is proportional to $[\mathrm{NO}]^{2}$. Typical experimental protocols utilize $\mu \mathrm{M}$ concentrations of [DAF], while in many biological tissues and conditions, $\mathrm{nM}$ levels of $\mathrm{NO}$ are anticipated. Thus, the second scenario is more likely to occur. Although the kinetic data was acquired from mixing DAF and NO donor in aerated buffered solutions, similar behavior may hold in biological tissues. Ideally, the applicability of Equation $2.8 \mathrm{a}$ or $2.8 \mathrm{~b}$ should be verified on a particular system and a method of analysis should be designed accordingly. For example, normalizing FI(t) with initial fluorescence may be required according to Equation $2.8 \mathrm{a}$ but not according to $2.8 \mathrm{~b}$ to yield data slopes independent of [DAF]. Nevertheless, phenomena such as uneven dye loading, motion artifacts or dye leakage can still affect fluorescence through their effect on coefficient $\alpha$.

Summary. The absence of reliable methods for quantifying NO in biological tissues has provided a significant obstacle in elucidating NO functions in biomedicine. NO sensitive fluorescent probes are ideally suited for such investigations by allowing 
spatio-temporal NO monitoring. Despite several drawbacks associated with the use of DAFs, these fluorescent probes have adequate sensitivity for assessing physiological levels of NO that are usually in the nanomolar range. The kinetic information provided in this study can assist in the development of calibration protocols that will enable us to quantify cellular NO levels in different systems and ultimately estimate endothelial derived NO production rates. Future studies should test the kinetic behavior in cellular systems and validate fluorescent based quantification of $\mathrm{NO}$ against other established methods (i.e. Chemiluminescence, electrochemical).

\subsection{Acknowledgments}

This work was funded by National Institutes of Health Grant SC1HL095101 (NT).

\subsection{References}

[1] S. Archer, Measurement of nitric oxide in biological models. FASEB 7 (1993) 349-360.

[2] M. Feelisch, Rassaf, T., Mnaimneh, S., Singh, N., Bryan, N.S., Jourd'Heuil, D., Kelm, M., Concomitant S-, N-, and heme-nitros(yl)ation in biological tissues and fluids: Implications for the fate of NO in vivo. Free Radic Biol Med 33 (2002) 1590-1596.

[3] M. Marzinzig, Nussler, A.K., Stadler, J., Marzinzig, E., Barthlen, W., Nussler, N.C., Beger, H.G., Morris, S.M. Jr., Bruckner, U.B., Improved methods to measure end products of nitric oxide in biological fluids: Nitrite, nitrate, and Snitrosothiols. Nitric Oxide 1 (1997) 177-189.

[4] J.E. Stern, Zhang, W., Cellular sources, targets and actions of constitutive nitric oxide in the magnocellular neurosectretory system of the rat. J Physiol 562 (2005) 725-744.

[5] M. Chvanov, Gerasimenko, O.V., Petersen, O.H., Tepikin, A.V., Calciumdependent release of NO from intracellular S-nitrosothiols EMBO 25 (2006) 3024-3032. 
[6] S.L. Hempel, Buettner, G.R., O'Maller, Y.Q., Wessels, D.A., Flaherty, D.M., Dihydrofluorescein diacetate is superior for detecting intracellular oxidants: Comparison with 2',7'-dichlorodihydrofluorescein diacetate, 5(and 6)-caroxy-2',7'dichlorodihydrofluorescein diacetate, and dihydrorhodamine 123. Free Radic Biol Med 27 (1999) 146-159.

[7] H. Kojima, Nakatsubo, N., Kikuchi, K., Kawahara, S., Kirino, Y., Nagoshi, H., Hirata, Y., Nagano, T., Detection and imaging of nitric oxide with novel fluorescent indicators: Diaminoflurosceins. Anal Chem 70 (1998) 2446-2453.

[8] H. Kojima, Sakurai, K., Kikuchi, K., Kawahara, S., Kirino, Y., Nagoshi, H., Hirata Y., Nagano, T.,, Development of a fluorescent indicator for nitric oxide based on the fluorescein chromophore. Chem Pharm Bull (Tokyo) 46 (1998) 373375.

[9] M.H. Lim, Lippard, S.J., Fluorescent nitric oxide detection by copper complexes bearing anthracenyl and dansyl fluorophore ligands. Inorg Chem 45 (2006) 89808989.

[10] E. Sasaki, Kojima, H., Nishimatsu, H., Urano, Y., Kikuchi, K., Hirata, Y., Nagano, T., Highly sensitive near-infrared fluorescent probes for nitric oxide and their application to isolated organs. J Am Chem Soc 127 (2005) 3684-3685.

[11] N. Soh, Katayama, Y., Maeda, M., A flurescent probe for monitoring nitric oxide production using a novel detection concept Analyst 126 (2001) 564-566.

[12] X.B. Ji, Hollocher, T.C., Mechanism for nitrosation of 2,3-diaminonaphthalene by Escherichia coli: Enzymatic production of NO followed by O2-dependent chemical nitrosation. Appl Environ Microbiol 54 (1988) 1791-1794.

[13] T. Nagano, Practical methods for detection of nitric oxide. Luminescence 14 (1999) 283-90.

[14] H. Kojima, Hirata, M., Kudo, Y., Kikuchi, K., Nagano, T., Visualization of oxygen-concentration-dependent production of nitric oxide in rat hippocampal slices during aglycemia. J Neurochem 76 (2001) 1404-1410.

[15] M.G. Espey, D.D. Thomas, K.M. Miranda, and D.A. Wink, Focusing of nitric oxide mediated nitrosation and oxidative nitrosylation as a consequence of reaction with superoxide. Proc Natl Acad Sci U S A 99 (2002) 11127-32.

[16] G. Nagy, Barcza, M., Gonchoroff, N., Phillips, P.E., and Perl, A., Nitric OxideDependent Mitochondrial Biogenesis Generates Ca 2+ Signaling Profile of Lupus T Cells. J Immunol 173 (2004) 3676-3683. 
[17] Y. Qian, Banerjee, S., Grossman, C.E., Amidon, W., Nagy, G., Barcza, M., Niland, B., Karp, D.R., Middleton, F.A., Bank, K., and Perl, A., Transaldolase deficiency influences the pentose phosphate pathway, mitochondrial homoeostasis and apoptosis signal processing. Biochem. J. 415 (2008) 123-134.

[18] E. Grossini, Molinari, C., Mary, D.A.S.G., Uberti, F., Caimmi, P.P., Surico, N., and Vacca, G., Intracoronary Genistein Acutely Increases Coronary Blood Flow in Anesthetized Pigs through beta-Adrenergic Mediated Nitric Oxide Release and Estrogenic Receptors. Endocrinology 149 (2008) 2678-2687.

[19] M. Nyberg, Mortensen, S.P., Thaning, P., Saltin, B., and Hellsten, Y., Interstitial and Plasma Adenosine Stimulate Nitric Oxide and Prostacyclin Formation in Human Skeletal Muscle. Hypertension 56 (2010) 1102-1108.

[20] E. Planchet, Kaiser, W.M., Nitric oxide (NO) detection by DAF fluorescence and chemiluminescence: A comparison using abiotic and biotic NO sources. J Exp Bot 57 (2006) 3043-3055.

[21] R. Berkels, Dachs, C., Roesen, R., Klaus, W., Simultaneous measurement of intracellular $\mathrm{Ca}(2+)$ and nitric oxide: A new method. Cell Calcium 27 (2000) 281286.

[22] N.S. Bryan, and Grisham, M.B., Methods to detect nitric oxide and its metabolites in biological samples. Free Radic Biol Med 43 (2007) 645-657.

[23] M. Broillet, O. Randin, and J. Chatton, Photoactivation and calcium sensitivity of the fluorescent $\mathrm{NO}$ indicator 4,5-diaminofluorescein (DAF-2): implications for cellular NO imaging. FEBS Lett 491 (2001) 227-32.

[24] H. Hong, J. Sun, and W. Cai, Multimodality imaging of nitric oxide and nitric oxide synthases. Free Radic Biol Med 47 (2009) 684-98.

[25] N. Nakatsubo, H. Kojima, K. Kikuchi, H. Nagoshi, Y. Hirata, D. Maeda, Y. Imai, T. Irimura, and T. Nagano, Direct evidence of nitric oxide production from bovine aortic endothelial cells using new fluorescence indicators: diaminofluoresceins. FEBS Lett 427 (1998) 263-6.

[26] D.L.H. Williams, Nitrosation reactions and the chemisty of nitric oxide, Elsevier B.V., Amsterdam, 2004.

[27] V.G. Kharitonov, Sundquist, A.R., Sharma, V.S., Kinetics of nitrosation of thiols by nitric oxide in the presence of oxygen. J Biol Chem 270 (1995) 28158-28164. 
[28] C. Griffiths, V. Wykes, T.C. Bellamy, and J. Garthwaite, A new and simple method for delivering clamped nitric oxide concentrations in the physiological range: application to activation of guanylyl cyclase-coupled nitric oxide receptors. Mol Pharmacol 64 (2003) 1349-56.

[29] M. Nyberg, S.P. Mortensen, P. Thaning, B. Saltin, and Y. Hellsten, Interstitial and plasma adenosine stimulate nitric oxide and prostacyclin formation in human skeletal muscle. Hypertension 56 (2010) 1102-8.

[30] P. Wardman, Fluorescent and luminescent probes for measurement of oxidative and nitrosative species in cells and tissues: Progress, pitfalls, and prospects. Free Radic Biol Med 43 (2007) 995-1022.

[31] A. Schrammel, A.C. Gorren, K. Schmidt, S. Pfeiffer, and B. Mayer, S-nitrosation of glutathione by nitric oxide, peroxynitrite, and $(*) \mathrm{NO} / \mathrm{O}(2)\left({ }^{*}\right)$. Free Radic Biol Med 34 (2003) 1078-88.

[32] A.A. Nedospasov, Is $\mathrm{N} 2 \mathrm{O} 3$ the main nitrosating intermediate in aerated nitric oxide (NO) solutions in vivo? If so, where, when, and which one? J Biochem Mol Toxicol 16 (2002) 109-20.

[33] A. Keszler, Zhang, Y., Hogg, N., Reaction between nitric oxide, glutathione, and oxygen in the presence and absence of protein: How are s-nitrosothiols formed? Free Radic Biol Med 48 (2010) 55-64.

[34] D. Jourd'heuil, F.L. Jourd'heuil, and M. Feelisch, Oxidation and nitrosation of thiols at low micromolar exposure to nitric oxide - Evidence for a free radical mechanism. Journal of Biological Chemistry 278 (2003) 15720-15726.

[35] A.B. Ross, Mallard, W. G., Helman, W. P., Buxton, G. V., Huie, R. E., Neta, P., NDRL-NIST Solution Kinetics Database: Ver. 3, Notre Dame Radiation Laboratory and National Institute of Standards and Technology, 1998.

[36] T.R. Rathel, Leikert, J.J., Vollmar, A.M, Dirsch, V.M., Application of 4,5diaminofluorescein to reliably measure nitric oxide released from endothelial cells in vitro. Biol Proced Online 5 (2003) 136-142. 


\section{Appendix 2.1}

\section{Mathematical model of the proposed kinetic mechanism:}

Applying the law of mass action to the first reaction scheme (i.e. Reactions 2.1-

2.4), yields four differential equations for the rates of change of $\mathrm{NO}, \mathrm{NO}_{2}^{\bullet}, \mathrm{N}_{2} \mathrm{O}_{3}$, and DAF-T as follows:

$$
\begin{aligned}
& \frac{\mathrm{d}[\mathrm{NO}]}{\mathrm{dt}}=-2 \mathrm{k}[\mathrm{NO}]^{2}\left[\mathrm{O}_{2}\right]-\mathrm{k}_{2}\left[\mathrm{NO}_{2}\right][\mathrm{NO}]+\mathrm{k}_{-2}\left[\mathrm{~N}_{2} \mathrm{O}_{3}\right] \\
& \frac{\mathrm{d}\left[\mathrm{NO}_{2}\right]}{\mathrm{dt}}=2 \mathrm{k}_{1}[\mathrm{NO}]^{2}\left[\mathrm{O}_{2}\right]-\mathrm{k}_{2}\left[\mathrm{NO}_{2}\right][\mathrm{NO}]+\mathrm{k}_{-2}\left[\mathrm{~N}_{2} \mathrm{O}_{3}\right] \\
& \frac{\mathrm{d}\left[\mathrm{N}_{2} \mathrm{O}_{3}\right]}{\mathrm{dt}}=\mathrm{k}_{2}\left[\mathrm{NO}_{2}\right][\mathrm{NO}]-\mathrm{k}_{-2}\left[\mathrm{~N}_{2} \mathrm{O}_{3}\right]-\mathrm{k}_{3}\left[\mathrm{~N}_{2} \mathrm{O}_{3}\right]-\mathrm{k}_{4}\left[\mathrm{~N}_{2} \mathrm{O}_{3}\right][\mathrm{DAF}] \\
& \frac{\mathrm{d}[\mathrm{DAF}-\mathrm{T}]}{\mathrm{dt}}=\mathrm{k}_{4}\left[\mathrm{~N}_{2} \mathrm{O}_{3}\right][\mathrm{DAF}]
\end{aligned}
$$

For the revised reaction mechanism, that assumes DAF activation by $\mathrm{NO}_{2}^{\bullet}$ (i.e. Reactions 2.1, 2.2, 2.3, and 2.5), Eqs. A.2.2-A.2.4 are modified to reflect this change.

$$
\begin{aligned}
& \frac{\mathrm{d}\left[\mathrm{NO}_{2}\right]}{\mathrm{dt}}=2 \mathrm{k}_{1}[\mathrm{NO}]^{2}\left[\mathrm{O}_{2}\right]-\mathrm{k}_{2}\left[\mathrm{NO}_{2}\right][\mathrm{NO}]+\mathrm{k}_{-2}\left[\mathrm{~N}_{2} \mathrm{O}_{3}\right]-\mathrm{k}_{5}\left[\mathrm{NO}_{2}\right][\mathrm{DAF}] \\
& \frac{\mathrm{d}\left[\mathrm{N}_{2} \mathrm{O}_{3}\right]}{\mathrm{dt}}=\mathrm{k}_{2}\left[\mathrm{NO}_{2}\right][\mathrm{NO}]-\mathrm{k}_{-2}\left[\mathrm{~N}_{2} \mathrm{O}_{3}\right]-\mathrm{k}_{3}\left[\mathrm{~N}_{2} \mathrm{O}_{3}\right] \\
& \frac{\mathrm{d}[\mathrm{DAF}-\mathrm{T}]}{\mathrm{dt}}=\mathrm{k}_{5}\left[\mathrm{NO}_{2}\right][\mathrm{DAF}]
\end{aligned}
$$


Differential equations can also describe the change in the $\mathrm{O}_{2}$ and DAF-FM concentrations. However, we can assume that due to the abundance of $\mathrm{O}_{2}$ its concentration will remain essentially constant while the total concentration of the dye is preserved. Eq. 2.5 can then estimate the concentration of DAF:

$[\mathrm{DAF}]=[\mathrm{DAF}]_{\mathrm{i}}-[\mathrm{DAF}-\mathrm{T}]$

where $[\mathrm{DAF}]_{\mathrm{i}}$ is the initial dye concentration.

An NO donor, with a relatively long half-life, can provide continuous release of NO over the duration of the experiment. To model this scenario, Eq. A.2.1 is modified to incorporate the NO release:

$\frac{\mathrm{d}[\mathrm{NO}]}{\mathrm{dt}}=\mathrm{S}-2 \mathrm{k}[\mathrm{NO}]^{2}\left[\mathrm{O}_{2}\right]-\mathrm{k}_{2}\left[\mathrm{NO}_{2}\right][\mathrm{NO}]+\mathrm{k}_{-2}\left[\mathrm{~N}_{2} \mathrm{O}_{3}\right]$

where $\mathrm{S}$ is the NO release rate given by:

$\mathrm{S}=2 \mathrm{k}_{\mathrm{d}}[$ NOdonor $] \exp ^{\left(-\mathrm{k}_{\mathrm{d}} \cdot \mathrm{t}\right)}$

$k_{d}$ is the dissociation rate constant of the NO donor and is inversely proportional to the half-life $\left(\mathrm{k}_{\mathrm{d}}=\ln 2 / \mathrm{t}_{1 / 2}\right)$.

Although simulations exhibited in Figure 2.1A were performed for the proposed kinetic mechanism, an identical simulation is achieved for the revised kinetic mechanism (not shown). Similarly, the simulated evolution of DAF-T over time follows comparable profiles for both kinetic mechanisms. Figure 2.2A shows simulations for the first kinetic mechanisms (Eq. A.2.2, A.2.3, A.2.4, A.2.9) assuming a $\mathrm{k}_{4}$ value of $4.0 \times 10^{8} \mathrm{M}^{-1} \mathrm{~s}^{-1}$. 


\section{Modeling parameters utilized:}

\begin{tabular}{|c|c|}
\hline Parameters & Values \\
\hline $\mathrm{k}_{1}$ & $3.15 \times 10^{6} \mathrm{M}^{-2} \mathrm{~s}^{-1}$ \\
\hline $\mathrm{k}_{2}$ & $1.1 \times 10^{9} \mathrm{M}^{-1} \mathrm{~s}^{-1}$ \\
\hline $\mathrm{k}_{-2}$ & $81000 \mathrm{~s}^{-1}$ \\
\hline $\mathrm{k}_{3}$ & $38130 \mathrm{~s}^{-1}$ \\
\hline $\mathrm{k}_{4}$ & $4.0 \times 10^{8} \mathrm{M}^{-1} \mathrm{~s}^{-1}$ \\
\hline $\mathrm{k}_{5}$ & $8.2 \times 10^{7} \mathrm{M}^{-1} \mathrm{~s}^{-1}$ \\
\hline $\mathrm{K}_{\mathrm{m}}$ & 4.27 \\
\hline$\left[\mathrm{O}_{2}\right]$ & $2.77 \times 10^{-4} \mathrm{M}$ \\
\hline
\end{tabular}

Table A.2.1 - Modeling parameters utilized [26] 
Chapter 3

APPROXIMATION OF NITRIC OXIDE RELEASE BY

ENDOTHELIAL CELLS UTILIZING DAF-FM 


\subsection{Abstract}

Endothelial derived NO production rates has been difficult to determine and current predicted values are widely ranging. Fluorescent microscopy is the most common method available in determining NO production because it allows real-time bioimaging. DAF-FM da is the most widely utilized cell permeable fluorescent indicator for in vitro NO research. We have previously performed a detailed kinetic analysis between DAF-FM and NO and have developed relationships describing DAF-FM fluorescent signals to NO concentrations at physiological conditions. These relationships were the driving forces in developing a calibration protocol for cellular systems. NO concentration values from maximum agonist stimulated Human Umbilical Vein Endothelial Cells (HUVECs) were obtained and values were further corroborated by chemiluminescence. Additional mathematical modeling was performed utilizing finite element methods (FEM) to approximate endothelial derived NO production rates. At maximum agonist stimulation, the average NO production rate was found to be

approximately $0.418 \pm 0.18 \mathrm{pmol} / \mathrm{cm}^{2} \mathrm{~s}$. Assuming an endothelial layer thickness of 4 $\mu \mathrm{m}$, this is equivalent to $1.05 \mu \mathrm{M} / \mathrm{s}$.

\subsection{Introduction}

Different methods for detecting endothelial derived NO have been utilized. However, fluorometric analysis is the only method available for intracellular NO studies [1]. The development of fluorescent probes in the recent years has demonstrated to be a sensitive and specific assay system that is gaining wide application to measure nitrogen oxides $[1,2,3,4,5,6,7]$. NO-reactive fluorescent indicators allow bio-imaging of NO 
with high spatiotemporal resolution, permitting investigation of two-dimensional NO production in real-time by digital fluorescence imaging, which is not possible with other NO detection methods [8]. These fluorescent probes may be used to measure nanomolar levels of NO generated under biological conditions [9]. Currently, one of the most widely used fluorescent probes, 4-amino-5-methylamino-2',7'-difluorescein diacetate (DAF-FM da), was also developed and utilized as an in vitro NO detection probe. DAFFM da is cell permeable and is virtually non-fluorescent, however, upon reaction with NO, a highly fluorescent benzotriazole is formed (DAF-FM T). Because the reaction between NO and DAF-FM is irreversible, the fluorescence intensity (FI) will not decrease.

Unlike many fluorescent indicators, DAF-FM da is not a ratiometric dye; therefore, calibration has been difficult due to factors such as dye leakage, uneven dye loading, and photobleaching $[10,11]$. More importantly, the actual kinetics of the reaction between NO and DAF-FM da has yet to be elucidated. Calibration of this dye can be beneficial in estimating cellular NO concentrations ([NO]) as well as determining endothelial derived NO production rates. We have previously developed kinetic equations to describe the relationship between change in fluorescent signal to actual NO concentration levels in physiological systems. These equations will provide the basis for the calibration protocol provided.

\subsection{Methods}

Chemicals and Materials. The base media for the HUVECs was phenol red free DMEM/F12k (Invitrogen) medium supplemented with $0.1 \mathrm{mg} / \mathrm{mL}$ of heparin and 0.03- 
$0.05 \mathrm{mg} / \mathrm{mL}$ ECGS from Sigma-Aldrich, 1\% penicillin/streptomycin from Invitrogen, and $10 \%$ fetal bovine serum (FBS) (ATCC; Manassas, VA). A base media free of phenol red was chosen because phenol red has been shown to interfere with fluorescent measurements. DAF-FM diacetate (da) (4-Amino-5-(N-methylamino)-3',6'bis(acetyloxy)-2',7'-difluoro-spiro[isobenzofuran-1(3H), ' 9 -[9H]xanthen]-3-one) $\quad$ was purchased from Molecular Probes. A $5 \mathrm{mM}$ stock solution of DAF-FM da was prepared in DMSO and diluted to working concentrations in a premade buffer prior to experimentation. $\quad N_{\omega}$-Nitro-L-arginine methyl ester hydrochloride (L-NAME) and agonists acetylcholine and bradykinin were purchased from Sigma-Aldrich. Calcimycin was purchased from Enzo Life Sciences (Farmingdale, NY). Final working concentrations of L-NAME, bradykinin, and acetylcholine were prepared in PBS (Invitrogen). Calcimycin was prepared by first dissolving in DMSO and further diluting with a premade buffer solution containing calcium $(2.5 \mathrm{mM})$.

A premade buffer solution was made. Table 3.1 below shows the components used and their corresponding concentrations. The Sodium Chloride $(\mathrm{NaCl})$, Calcium Chloride $\left(\mathrm{CaCl}_{2}\right)$, Magnesium Sulfate $\left(\mathrm{MgSO}_{4}\right)$, Potassium Phosphate $\left(\mathrm{KH}_{2} \mathrm{PO}_{4}\right)$, and HEPES were purchased from Sigma-Aldrich. The Potassium Chloride $(\mathrm{KCl})$ and Sodium Carbonate $\left(\mathrm{NaHCO}_{3}\right)$ were purchased from Fisher Scientific (Pittsburgh, PA). Finally, the Glucose was purchased from Invitrogen. All components were added to 1 liter of deionized water and brought to a $\mathrm{pH}$ of 7.4. 


\begin{tabular}{|c|c|}
\hline Buffer Component & Final Concentration (mM) \\
\hline $\mathrm{NaCl}$ & 118.8 \\
\hline $\mathrm{KCl}$ & 5 \\
\hline $\mathrm{CaCl}_{2}$ & 2.5 \\
\hline $\mathrm{MgSO}_{4}$ & 1.2 \\
\hline $\mathrm{KH}_{2} \mathrm{PO}_{4}$ & 1.2 \\
\hline $\mathrm{NaHCO}_{3}$ & 2.7 \\
\hline $\mathrm{HEPES}$ & 20 \\
\hline Glucose & 10.1 \\
\hline
\end{tabular}

Table 3.1 - Individual components and concentrations of premade buffer solution.

Cell Culturing and Cell Experiment Preparation. Human Umbilical Vein Endothelial Cells (HUVEC) (Cell Applications; San Diego, CA) were used in experimental work for determining endogenously produced NO. When purchased, they were received at passage 1. Cell expansion was performed until cells reached passage 4, where they were then frozen down. All cellular experiments were performed at this passage. Appendix 3.1 and 3.2 give a detailed explanation of the cell culturing methods for feeding and passaging of cells respectively. Prior to experimentation, cells were seeded into $35 \mathrm{~mm}^{2}$ culture dishes and cultured until an $80-90 \%$ confluency was reached. For both the fluorescent and chemiluminescent experiments, cells were starved with serum-free media for 1 hour at $37^{\circ} \mathrm{C}, 5 \% \mathrm{CO}_{2}$ prior to stimulation. This was done to ensure that any residual FBS within the cells was removed. FBS has been shown to interfere with fluorescent measurements. In addition, the starvation period is necessary to ensure low basal levels of NO production.

Fluorescent Measurements. After the 1 hour incubation period with serum-free media, cells were rinsed 3 times with the premade buffer solution. A solution of $5 \mu \mathrm{M}$ 
DAF-FM da was prepared and added to the well plates. For control experiments using LNAME in blocking agonist stimulation, $500 \mu \mathrm{M}$ of L-NAME was added to the control wells with the DAF-FM da. The well plates were placed back into the incubator for 30 minutes at $37^{\circ} \mathrm{C}, 5 \% \mathrm{CO}_{2}$. Following this incubation period, the cells were again rinsed 3 times with the premade buffer solution. A final incubation with just buffer was done for 30 minutes to ensure complete esterification of the DAF-FM da. An automated fluorescent microscope (Olympus 1X-81) with an incorporated filter wheel was utilized in the detection of DAF-FM fluorescence in cells. The excitation and emission wavelength selected were $495 \mathrm{~nm}$ and $515 \mathrm{~nm}$ respectively, with a bandwidth of $10 \mathrm{~nm}$. During data collection, a shutter was used to control the exposure time of light given to the DAF-FM sample. An exposure time length of $20 \mathrm{~ms}$ was chosen to avoid over exposure of light to the sample for each frame collected. The experiment duration time was 15 minutes, sampling every 30 seconds. The objective was set to $10 \mathrm{X}$ for experimental purposes. Connected to the microscope was a camera (Retiga Q EXi), which enabled image acquisition. The information acquired was retrieved and analyzed using IPLab 3.6.3 (Scanalytics, VA). After experimental results were obtained in IPLab, manual selections of regions of interest were selected. One region selected to act as a normalizing region was of the background. The other regions selected were cells within the field of view. The fluorescent data from these regions were average for each frame collected and final averaged data was subtracted with the corresponding background region at each time point. This data was then normalized with the last fluorescent reading, yielding values from $0-1$. Similar to the NO solution experiments, the slopes, and ultimately $\beta_{\max }$, of the normalized fluorescent curves were determined. 
Chemiluminescent Measurements. After incubation with serum-free media, the cells were removed and rinsed 3 times with PBS. Agonist stimulants were then added to the cells, and well plates were incubated for 30 minutes to ensure complete release of NO from within the cells. The detection of [NO] released from cells after agonist stimulation was done via chemiluminescence. A nitric oxide analyzer (NOA Sievers 280i; Boulder, $\mathrm{CO}$ ) in conjunction with NO Analysis ${ }^{\mathrm{TM}}$ Software (Ionics; Boulder, CO) was utilized for data collection. A triiodide reducing solution was prepared by combining $0.4 \mathrm{~g}$ of Sodium Iodide (NaI) (Sigma-Aldrich), $0.26 \mathrm{~g}$ of Iodine $\left(\mathrm{I}_{2}\right)$ chips (Acros Organics; Waltham, MA), $8 \mathrm{~mL}$ of distilled water, and $28 \mathrm{~mL}$ of glacial acetic acid (SigmaAldrich). The solution was placed on a magnetic stirrer for 30-40 minutes to ensure components were completely dissolved. NO has a very short half-life and quickly reacts with $\mathrm{O}_{2}$ to form nitrite, the purpose of this reducing agent is to convert nitrite back to NO where it can then be detected by the NOA. An initial standard calibration curve was obtained using known concentrations of nitrite solutions before cell samples were analyzed, where only the supernatants of the cell solutions are analyzed. During this incubation period, a standard curve was obtained using known concentrations ( $25 \mu \mathrm{M}-1$ $\mathrm{mM})$ of Sodium Nitrite $\left(\mathrm{NaNO}_{2}\right)$ (Sigma-Aldrich). At the end of the incubation period, the supernatant above the cells was collected and analyzed in the NOA. The resulting values obtained were given in picomoles, therefore calculations were performed to yield concentration values based on the volume injected into the detecting system.

FEM Model. Finite Element Method (FEM) allows 2D modeling of the presented cellular system. With this approach, we can estimate endothelial derived NO production 
rates from the HUVECs upon agonist stimulation based on the [NO] values achieved experimentally. A diffusion reaction model was developed to examine changes in NO concentration with respect to time and spatial position. The following equation was utilized for the diffusion model:

$$
\frac{\partial \mathrm{C}}{\partial \mathrm{t}}+\nabla(-\mathrm{D} \nabla \mathrm{C})=\mathrm{R}
$$

where $\mathrm{C}$ is the concentration of NO, D is the diffusivity of NO $\left(3.3 \times 10^{-5} \mathrm{~cm}^{2} \cdot \mathrm{s}^{-1}\right)$, and R is the rate of NO consumption by the rate limiting oxidation reaction of $\mathrm{NO}$ $\left(\mathrm{R}=4 \mathrm{k}_{1}[\mathrm{NO}]^{2}\left[\mathrm{O}_{2}\right] ; \mathrm{k}_{1}=3.15 \times 10^{6} \mathrm{M}^{-2} \mathrm{~s}^{-1},\left[\mathrm{O}_{2}\right]=2.77 \times 10^{-4} \mathrm{M}\right)$. A semi infinite boundary condition was applied which assumes a zero flux at one end. Initial production rate values were chosen to reach experimentally derived NO concentration values utilizing the following flux equation where $\mathrm{N}$ is the Flux value.

$$
\mathrm{N}=-\mathrm{D} \nabla \mathrm{C}
$$

\subsection{Results}

Standard Curve by NO Donors. NO detection within cells varies in comparison to NO detection in solution. Therefore before experiments were performed to determine endogenously produced NO from HUVECs, sets of experiments were performed with NO donors to yield a standard curve $(n=4)$. Known concentrations of SPER/NO ranging from $50-750 \mu \mathrm{M}$ were used with $5 \mu \mathrm{M}$ DAF-FM da loaded HUVECs. The FI curves from the cells treated with SPER/NO were recorded and the corresponding slopes $\left(\beta_{\max }\right)$ were determined. Figure 3.1 below shows log-log plots of $[\mathrm{SPER} / \mathrm{NO}]$ and $\beta_{\max }$. 
Analysis of the relationships between SPER/NO and $\beta_{\max }$ showed noticeable differences depending on [SPER/NO]. At the lower range of [SPER/NO] $(5-25 \mu \mathrm{M})$, a linear fitting of the log-log data shows a slope of $1.1 \pm 0.3(\mathrm{p}=0.7512)$ (Figure 3.1A). Contrastingly, the relationship between SPER/NO and $\beta_{\max }$ at higher [SPER/NO] (100 $750 \mu \mathrm{M}$ ) yielded a slope of $0.4 \pm 0.2$ (Figure 3.1B). Statistical analysis showed that this value was statistically different than 1 with $p=0.0012$ and closer to 0.5 . Therefore, for lower values of $[\mathrm{SPER} / \mathrm{NO}], \beta_{\max }$ is linearly proportional to SPER/NO with the slope indicating a 1:1 ratio. Thus meaning at this $[\mathrm{SPER} / \mathrm{NO}]$ range, $\beta_{\max }$ is proportional to $\mathrm{NO}^{2}$. Alternatively, at higher values of [SPER/NO], $\beta_{\max }$ is shown to be proportional to approximately SPER/NO $\mathrm{NO}^{1 / 2}$. This would conclude that $\beta_{\max }$ is proportional to $\mathrm{NO}$ at higher $[\mathrm{SPER} / \mathrm{NO}]$.

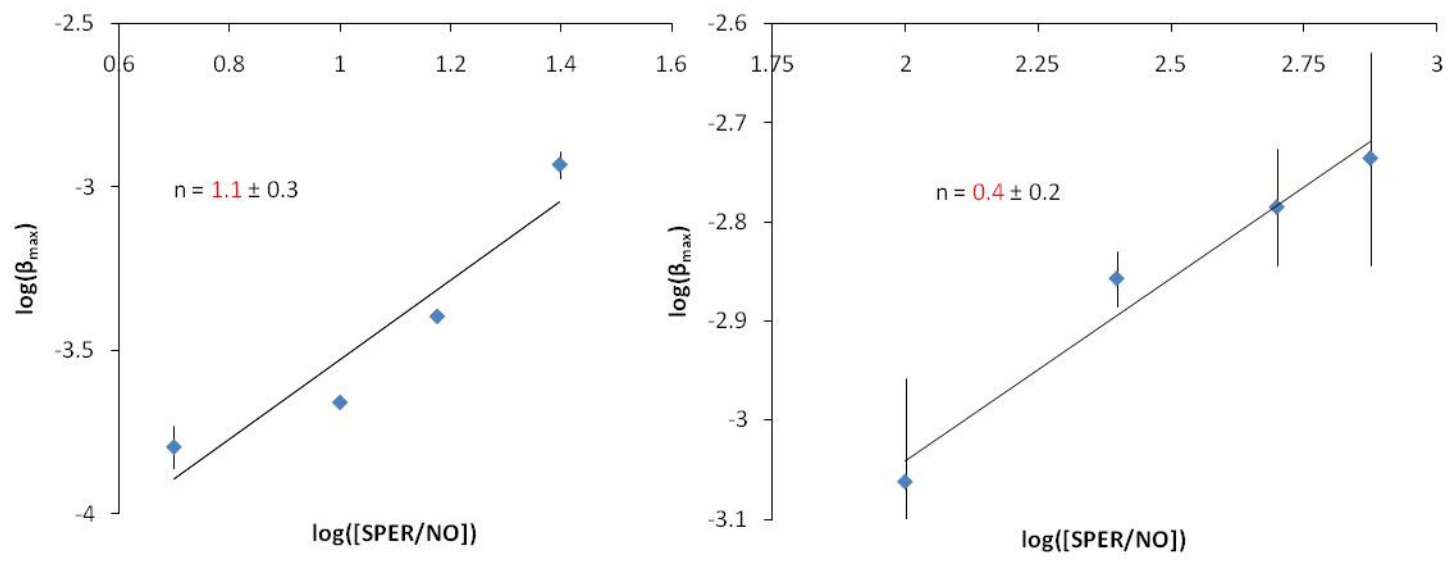

Figure 3.1 - Data from standard curve experiments $(n=4)$ presented as log-log plots of [SPER/NO] and $\beta_{\max }$. (A) Values from smaller [SPER/NO] ranging from $5-25 \mu \mathrm{M}$. Linear fit yields a slope of $1.1 \pm 0.3$. (B) Values from larger [SPER/NO] ranging from $100-750 \mu \mathrm{M}$. Linear fit yields a slope of $0.4 \pm 0.2$. 
The standard calibration curve will be used to estimate endogenous [NO] from HUVECs stimulated by agonists given by the resulting FI curve obtained. Depending on $\beta_{\max }$, or slope values, an approximate $[\mathrm{NO}]$ can be estimated.

Agonist Stimulated Fluorescent Detection. HUVECs loaded with DAF-FM da were stimulated with three different agonists: acetylcholine, calcimycin, and bradykinin. To ensure that the NO detected by the increase in DAF-FM fluorescence was directly attributed to the agonists applied, control trials were performed with the presence of LNAME. L-NAME is known to inhibit vasorelaxation induced by agonists. $\beta_{\max }$ from the FI curves was determined, averaged, and shown in Figure $3.2(\mathrm{n}=3)$.

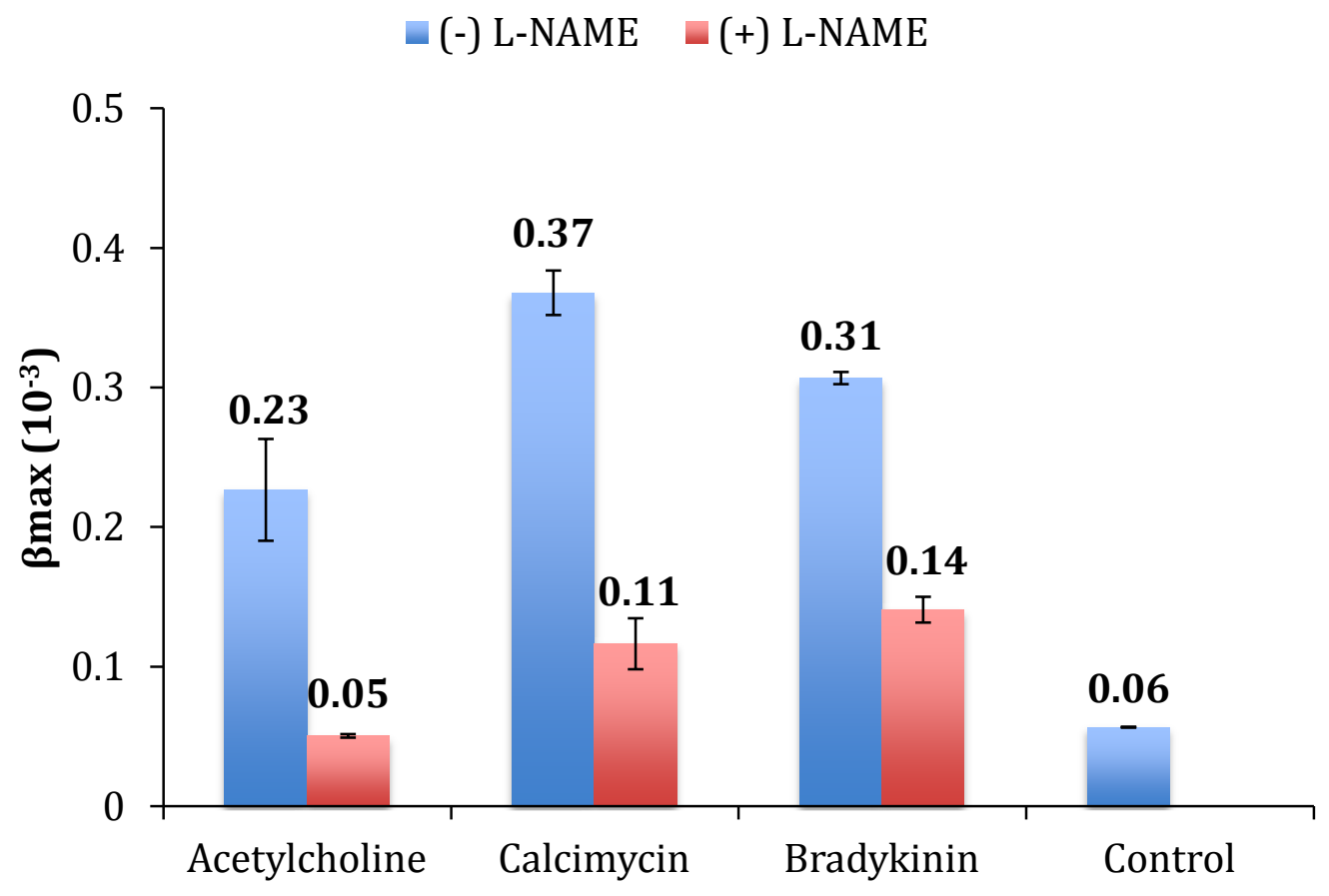

Figure 3.2 - Estimated $\beta_{\max }$ values obtained from HUVECs after agonist stimulation, with and without incubation with L-NAME.

From the $\beta_{\max }$ values given above for the different agonists without L-NAME, it is clear that the cells responded to the stimuli and endogenously produced $\mathrm{NO}$, as can be 
seen in comparison to the control with no stimulation. Statistical analysis shows that the HUVEC's responses from acetylcholine, calcimycin, and bradykinin were significantly different from the control response with $\mathrm{p}=9.6 \times 10^{-3}, 4.06 \times 10^{-4}$, and $5.59 \times 10^{-7}$ respectively. In addition, statistics comparing the agonist response in the HUVECs with and without incubation with L-NAME showed a significant reduction in signal when LNAME was present $\left(\mathrm{p}=8.5 \times 10^{-3}, 4.9 \times 10^{-4}\right.$, and $8.4 \times 10^{-5}$ respectively).

Agonist Stimulated Chemiluminescent Detection. Chemiluminescence was performed to quantify the NO generated from the HUVECs after agonist stimulation. Figure 3.3A displays the [NO] achieved using the different agonists. The concentrations achieved after stimulation with acetylcholine, calcimycin, and bradykinin were significantly different than the control value $(\mathrm{p}=0.02,0.03$, and 0.001 respectively). To analyze the actual [NO] achieved due to agonist stimulation, the baseline value was set to the average control value. Figure 3.3B displays the [NO] directly attributed to stimulation by the agonists. It was noted that at maximal agonist concentrations, calcimycin and bradykinin induced more overall NO production than acetycholine did. 

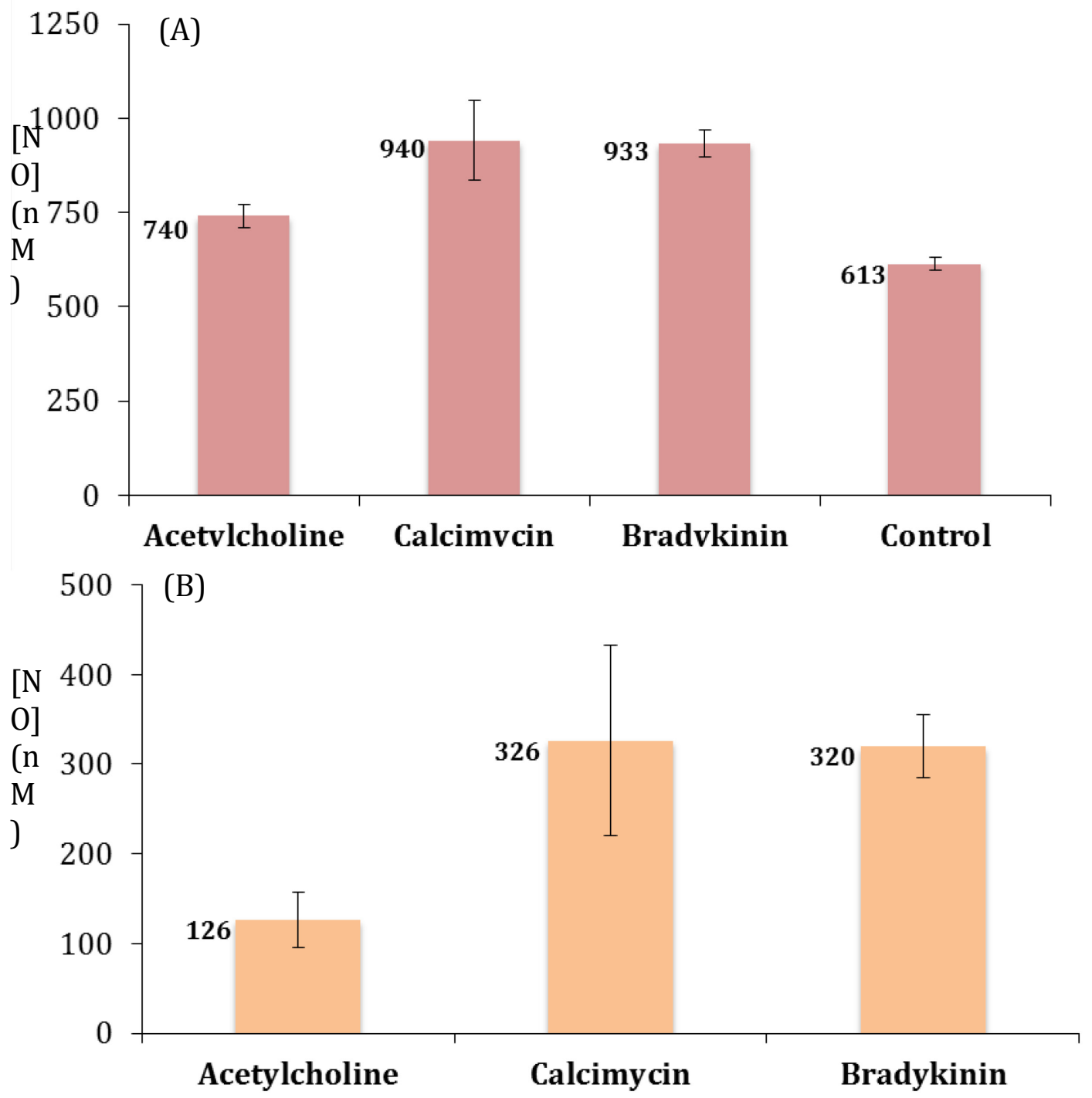

Figure 3.3 - Chemiluminescent data from HUVECs after agonist stimulation. (A) Overall data shown in comparison to control without agonist stimulation (B) Normalized [NO] obtained from agonist stimulation.

Modeling Results. To reach the NO concentration values determined experimentally, several different flux rates were investigated within the FEM model. Figure 3.4 shows the output generated from the model incorporating a production rate of $1.0 \mathrm{pmol} / \mathrm{cm}^{2} \mathrm{~s}$. A very fine mesh was utilized in the investigation area which yielded a NO concentration of $616.9 \mathrm{nM}$. A range of production rate values between $0.1-1.0$ $\mathrm{pmol} / \mathrm{cm}^{2}$ s generated NO levels that were experimentally achieved. 


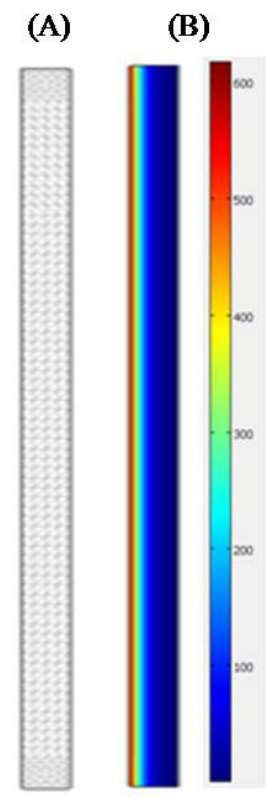

Figure 3.4 - FEM analysis results. (A) A very fine mesh was utilized for the investigation area yielding (B) NO concentration levels at a production rate of 1.0 $\mathrm{pmol} / \mathrm{cm}^{2} \mathrm{~s}$. Maximum NO levels achieved at this production rate was $616.9 \mathrm{nM}$.

\subsection{Discussion}

As discussed in the previous chapter, the differential equation describing the rate formation of DAF-FM $\mathrm{T}$ yields two limiting cases depending on [DAF-FM] and $\mathrm{K}_{\mathrm{m}}^{\prime}[\mathrm{NO}]$. From Figure 3.1, this observation is evident. The dependence in Figure 3.1A exhibits when $[\mathrm{DAF}-\mathrm{FM}]>\mathrm{K}_{\mathrm{m}}^{\prime}[\mathrm{NO}]$ and Figure $3.1 \mathrm{~B}$ displays when $[\mathrm{DAF}-\mathrm{FM}]<<$ $\mathrm{K}_{\mathrm{m}}^{\prime}[\mathrm{NO}]$. Under physiological conditions, or for estimations of endogenously produced NO, the case where $[\mathrm{DAF}-\mathrm{FM}]>\mathrm{K}_{\mathrm{m}}^{\prime}[\mathrm{NO}]$ is more appropriate for comparison. The $\beta_{\max }$ values from the FI curves determined from agonist stimulation on the HUVECs in comparison to the standard calibration data, fell into this region. [NO] achieved after agonist stimulation was determined from the $\beta_{\max }$ values. A MATLAB fitting of an 
equation of the standard calibration data for small values of NO yielded the following equation.

$$
\beta_{\text {max }}=\alpha[\mathrm{NO}]^{2}
$$

The estimated value of $\alpha$ was found to be $2.02 \times 10^{-6}$. The function given in Equation 3.3 allows for estimations of [NO] from cellular experiments with agonist stimulations. Figure 3.5 shows the fitting of the equation to the standard calibration data. The $\mathrm{R}^{2}$ value for this fit was 0.973 , ensuring that Equation 3.3 can be used in estimating [NO] from $\beta_{\max }$ values. The values determined were in the nanomolar range, which was expected especially for endogenously produced NO. In addition, the presence of L-NAME significantly hindered the production of NO.

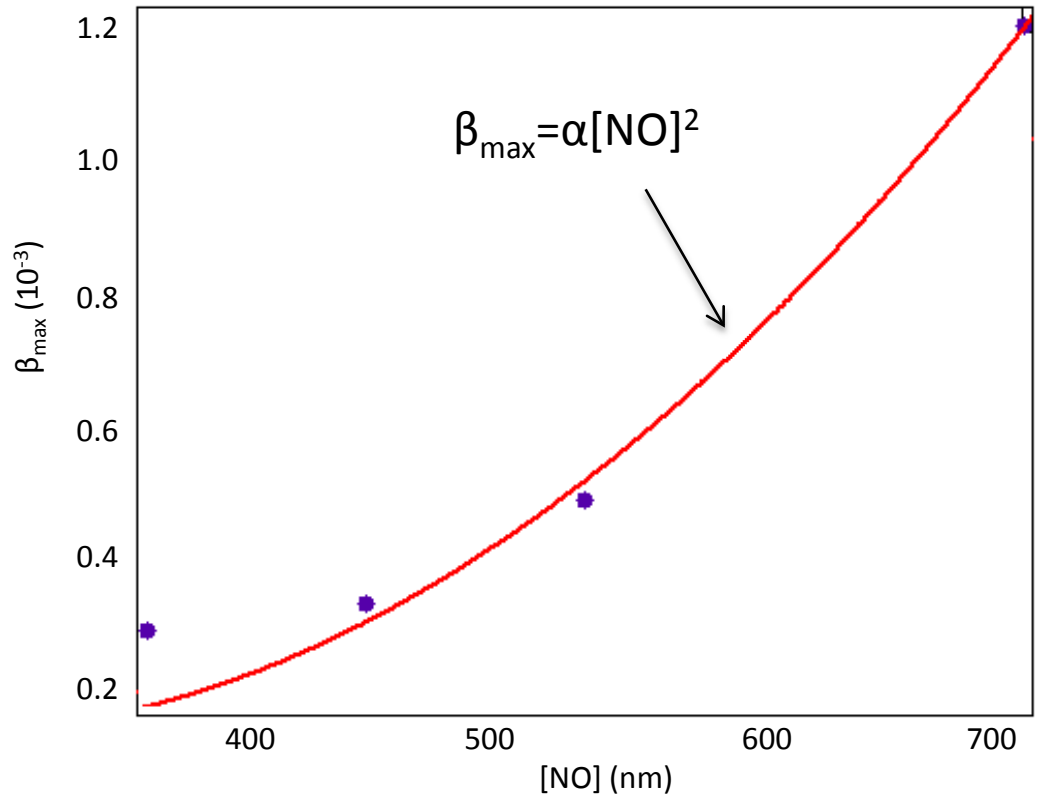

Figure 3.5 - Standard calibration data fitted to modeled equation to enable estimations of $[\mathrm{NO}]$ from endogenously produced sources. 
Table 3.2 below summarizes the $\beta_{\max }$ and corresponding [NO] due to agonist stimulation of HUVECs with and without incubation with L-NAME.

\begin{tabular}{|c|c|c|}
\hline Agonist & $\boldsymbol{\beta}_{\max } \mathbf{( 1 0}^{-\mathbf{3}} \mathbf{)}$ & {$[\mathbf{N O}] \mathbf{( n M )}$} \\
\hline Control & 0.057 & 168 \\
\hline Acetylcholine & 0.23 & 335 \\
\hline Acetylcholine + L-NAME & 0.051 & 158 \\
\hline Calcimycin & 0.37 & 427 \\
\hline Calcimycin + L-NAME & 0.11 & 240 \\
\hline Bradykinin & 0.31 & 390 \\
\hline Bradykinin + L-NAME & 0.14 & 264 \\
\hline
\end{tabular}

Table 3.2 - $\beta_{\max }$ and corresponding [NO] from HUVECs due to agonist stimulation.

Summary. This study was successful in stimulating HUVECs with agonists to induce endogenous NO production. This study was also successful in attempting to develop a calibration protocol of DAF-FM in a cellular system based on the revised kinetic mechanism developed in the previous chapter. A calibration curve of DAF-FM with NO donors was obtained as a standard to comparing FI signals attained from stimulated cells. Similarly to solution experiments, the slope, or $\beta_{\max }$, of the FI curve achieved by activated DAF-FM, was used in determining [NO]. As determined before, the rate equation developed for DAF-FM T formation yielded two cases in which the FI signal would differ upon experimental conditions. When considering experimental setups that are within physiological conditions (i.e. nanomolar levels of NO), case II of the rate equation must be utilized (i.e. $[\mathrm{DAF}-\mathrm{FM}] \gg \mathrm{K}_{\mathrm{m}}^{\prime}[\mathrm{NO}]$ ). FI signals obtained from agonist stimulated HUVECs were compared to the standard curve and [NO] values were determined. These values were corroborated with chemiluminescence readings. Findings from this study provided a capable protocol in utilizing DAF-FM in determining [NO] from cellular systems. 
To achieve the NO concentration levels at maximum agonist stimulation, the average production rate was found to be $0.418 \pm 0.18 \mathrm{pmol} / \mathrm{cm}^{2} \mathrm{~s}$. Assuming an endothelial layer thickness of $4 \mu \mathrm{m}$, this is equivalent to $1.05 \mu \mathrm{M} / \mathrm{s}$. This production rate value was noted to be within the same range as previously observed. This group also utilized HUVECs and detected NO using chemiluminescence [12].

\subsection{Acknowledgements}

This work was funded by National Institutes of Health Grant SC1HL095101 (NT).

\subsection{References}

[1] N. Soh, et al., "A fluorescent probe for monitoring nitric oxide production using a novel detection concept," Analyst, vol. 126, pp. 564-6, May 2001.

[2] M. Chvanov, et. al.,, "Calcium-dependent release of NO from intracellular Snitrosothiols," EMBOJ, vol. 25, pp. 3024-3032, 2006.

[3] S. L. Hempel, et. al., "Dihydrofluorescein diacetate is superior for detecting intracellular oxidants: comparison with 2',7'-dichlorodihydrofluorescein diacetate, 5(and 6)-carboxy-2',7'-dichlorodihydrofluorescein diacetate, and dihydrorhodamine 123," Free Radic Biol Med, vol. 27, pp. 146-159, 1999.

[4] H. Kojima, et al., "Development of a fluorescent indicator for nitric oxide based on the fluorescein chromophore," Chem Pharm Bull (Tokyo), vol. 46, pp. 373-5, Feb 1998.

[5] H. Kojima, et al., "Visualization of oxygen-concentration-dependent production of nitric oxide in rat hippocampal slices during aglycemia," $J$ Neurochem, vol. 76, pp. 1404-10, Mar 2001.

[6] M. H. Lim and S. J. Lippard, "Fluorescent nitric oxide detection by copper complexes bearing anthracenyl and dansyl fluorophore ligands," Inorg Chem, vol. 45, pp. 8980-9, Oct 302006.

[7] E. Sasaki, et al., "Highly sensitive near-infrared fluorescent probes for nitric oxide and their application to isolated organs," J Am Chem Soc, vol. 127, pp. 3684-5, Mar 232005. 
[8] H. Kojima, et al., "Detection and imaging of nitric oxide with novel fluorescent indicators: diaminofluoresceins," Anal Chem, vol. 70, pp. 2446-53, Jul 11998.

[9] X. B. Ji and T. C. Hollocher, "Mechanism for nitrosation of 2,3diaminonaphthalene by Escherichia coli: enzymatic production of NO followed by O2-dependent chemical nitrosation," Appl Environ Microbiol, vol. 54, pp. 1791-4, Jul 1988.

[10] A. Balcercyk, et. al.,, "On the specificity of 4-amino-5-methylamino-2',7'difluorofluorescein as a probe for nitric oxide," Free Radic Biol Med, vol. 39, pp. 327-335, 2005.

[11] M. Broillet, et al., "Photoactivation and calcium sensitivity of the fluorescent NO indicator 4,5-diaminofluorescein (DAF-2): implications for cellular NO imaging," FEBS Lett, vol. 491, pp. 227-32, Mar 2001.

[12] S. Potdar and M. Kavdia, "NO/peroxynitrite dynamics of high glucose-exposed HUVECs: chemiluminescent measurement and computational model," Microvasc Res, vol. 78, pp. 191-8, Sep 2009. 


\section{Appendix 3.1}

\section{Purpose}

This procedure describes the steps in changing the media of the cell cultures. The cells in consideration are HUVEC (Human Umbilical Vascular Endothelial Cells).

\subsection{Equipment \& Materials}

Equipment

HEPA-Filtered Laminar Flow Biological Safety Cabinet (LFBSC)

Incubator $37^{\circ} \mathrm{C}, 5 \% \mathrm{CO}_{2}$

Refrigerator at $2^{\circ} \mathrm{C}$ to $8^{\circ} \mathrm{C}$

Waterbath at $35^{\circ} \mathrm{C}$ to $39^{\circ} \mathrm{C}$

$\underline{\text { Materials }}$

Pipette

Pipette tips

Waste Container (150mL beaker)

$70 \%$ Ethanol

Kimwipes

\section{$2.0 \quad$ Reagents}

\begin{tabular}{|l|l|l|l|}
\hline Description & Catalog \# & Lot \# & Expiration Date \\
\hline Supplemented Media & & \\
\hline
\end{tabular}

3.0 Technician Signature Log

\begin{tabular}{|l|l|l|}
\hline Print Name & Signature & Initials \\
\hline & & \\
\hline & & \\
\hline & & \\
\hline & & \\
\hline
\end{tabular}

\subsection{Procedure}

4.1 Safety Precautions:

Biosafety Level 2 Procedures should be followed. Good aseptic technique should be used during all procedures which include wearing appropriate clothing (i.e.- lab coats and gloves) at all times inside the hood and spraying hands with alcohol anytime they are re-entered inside the hood. 
All work and opening of sterile materials or cell cultures must be done inside a HEPA filtered, laminar flow biological safety cabinet (LFBSC).

4.2 Hood cleared and cleaned by (Initial/date):

4.3 Preparation steps before media change

4.3.1 Turn on water bath and flow hood.

4.3.2 Remove aliquoted amount of supplemented media from refrigerator and place into the water bath. (Amount of media required depends on the amount of cell cultures needed to be fed.) NOTE: For flasks, the approximate amount of media needed follows the ratio of $5 \mathrm{~mL}$ per $25 \mathrm{~cm}^{2}$. For 24 well plates, $1 \mathrm{~mL}$ is needed per well.

4.3.3 Note the confluency of the cultures and record in notebook.

Completed by (Initials/Date) Verified by (Initials/Date)

4.4 Changing Media (To be completed after supplemented media is warm)

4.4.1 Spray down flow hood with $70 \%$ ethanol. Wipe down with kimwipes.

4.4.2 Spray down and place the following contents inside the hood: Cell cultures, waste bucket, aliquoted supplemented media, pipette, and appropriate pipette tips.

4.4.3 For flasks: Keep in an upright position, loosen the caps but do not completely open them until ready for use. Discard old media into the waste bucket by slowly pouring. Be careful not to cause splashing as contamination might occur.

4.4.4 For 24 well plates: Remove old media using a sterile pipette. Discard old media into the waste bucket.

4.4.5 Using a pipette, pull up the appropriate amount of media from aliquot and place into the flask or well plate. For the flasks, close the tops tightly.

4.4.6 Place cell cultures back into the incubator.

4.4.7 Remove all materials out of the hood and replace in their appropriate locations.

4.4.8 Spray and wipe down the hood when completed.

4.4.9 Discard of waste into the appropriate waste container.

Completed by (Initials/Date) Verified by (Initials/Date)

4.5 Further feeding considerations

4.5.1 Changing of media should be done every 48-72 hours. 


\section{Appendix 3.2}

\subsection{Purpose}

This procedure describes the steps in harvesting the cell cultures. The cells in consideration are HUVEC (Human Umbilical Vascular Endothelial Cells).

\subsection{Equipment \& Materials}

Equipment

HEPA-Filtered Laminar Flow Biological Safety Cabinet (LFBSC)

Incubator $37^{\circ} \mathrm{C}, 5 \% \mathrm{CO}_{2}$

Refrigerator at $2^{\circ} \mathrm{C}$ to $8^{\circ} \mathrm{C}$

Waterbath at $35^{\circ} \mathrm{C}$ to $39^{\circ} \mathrm{C}$

Centrifuge

$\underline{\text { Materials }}$

Pipette

Pipette tips

Waste Container (150mL beaker)

$70 \%$ Ethanol

Kimwipes

Conical tubes

\subsection{Reagents}

\begin{tabular}{|l|l|l|l|}
\hline Description & Catalog \# & Lot \# & Expiration Date \\
\hline Supplemented Media & & & \\
\hline PBS & & & \\
\hline Trypsin & & & \\
\hline
\end{tabular}

Completed by (Initials/Date) Verified by (Initials/Date)

\subsection{Technician Signature Log}

\begin{tabular}{|l|l|l|}
\hline Print Name & Signature & Initials \\
\hline & & \\
\hline & & \\
\hline
\end{tabular}

5.0 Procedure

5.1 Safety Precautions: 
Biosafety Level 2 Procedures should be followed. Good aseptic technique should be used during all procedures which include wearing appropriate clothing (i.e.- lab coats and gloves) at all times inside the hood and spraying hands with alcohol anytime they are re-entered inside the hood. All work and opening of sterile materials or cell cultures must be done inside a HEPA filtered, laminar flow biological safety cabinet (LFBSC).

5.2 Hood cleared and cleaned by (Initial/date):

5.3 Preparation steps before harvesting

5.3.1 Turn on water bath and flow hood. Remove aliquoted amount of supplemented media and trypsin from refrigerator and place into the water bath.

5.3.2 Note the confluency of the cultures and record in notebook. Refer to notebook

Completed by (Initials/Date) ___ Verified by (Initials/Date)

5.4 Harvesting of cell cultures (To be completed after the trypsin and supplemented media are warm)

5.4.1 Spray down flow hood with $70 \%$ ethanol. Wipe down with kimwipes.

5.4.2 Spray down and place the following contents inside the hood: Cell cultures, waste bucket, aliquoted supplemented media, aliquoted typsin, PBS, conical tubes with rack, pipette, and appropriate pipette tips.

5.4.3 For flasks:

5.4.3.1 Keep in an upright position, loosen the caps but do not completely open them until ready for use. Discard old media into the waste bucket by slowly pouring. Be careful not to cause splashing as contamination might occur.

5.4.3.2 Add PBS to the flasks. (NOTE: The amount of PBS added follows the ratio of $5 \mathrm{~mL}$ per $25 \mathrm{~cm}^{2}$.)

5.4.3.3 Gently rock the flasks back and forth for approximately 1 minute being careful not to get any liquid in the filter of the cap.

5.4.3.4 Discard waste the same way described in step 5.4.3.1.

5.4.3.5 Repeat steps 5.4.3.2-5.4.3.4 to ensure the cells have been properly rinsed.

5.4.3.6 Add trypsin to the flasks (NOTE: Approximate amount of trypsin to be used follows the ratio of $1-2 \mathrm{~mL}$ per $25 \mathrm{~cm}^{2}$.)

5.4.3.7 Place flasks into the incubator. Check flasks frequently under the microscope to ensure that all cells have lifted off the flask. Timing will vary depending on the confluency. Light tapping on the flask will assist in removing the cells from the flask. (NOTE: Do not leave the cells in trypsin for longer time than needed as it will damage the cells) 
5.4.3.8 Once cells have been completely lifted off the flask, place back into the hood using proper aseptic techniques described above.

5.4.3.9 To quench the trypsin add the equivalent amount of media. Gently rock to mix well.

5.4.3.10 Using a pipette of the appropriate volume, remove the cell suspension and place into a conical tube.

5.4.3.11 Rinse once again by adding the same amount of media used to quench. Rock gently and remove suspension as described in the previous step. (NOTE: As a check, look at the flasks or well plates under a microscope to ensure that all the cells have been removed)

5.4.3.12 Place the conical tubes into the centrifuge and spin for 7 minutes at $1200 \mathrm{rpm}$ at $25^{\circ} \mathrm{C}$.

5.4.3.13 Carefully remove the conical tubes from the centrifuge to avoid disrupting the cell pellet and place into the hood.

5.4.3.14 Discard the supernatant leaving only the cell pellet.

5.4.3.15 Resuspend the cell pellet with an appropriate amount of media.

5.4.3.16 Cells are now ready for further use.

5.4.4 For 24 well plates: Remove old media using a sterile pipette. Discard old media into the waste bucket.

5.4.4.1 Rinse the wells by adding $1 \mathrm{~mL}$ of PBS to each well.

5.4.4.2 Gently rock the well plates to ensure that the cells have been rinsed sufficiently and any residual media is cleaned.

5.4.4.3 Remove waste as described in step 5.4.4.

5.4.4.4 Repeat steps 5.4.4.1-5.4.4.3.

5.4.4.5 Add trypsin to each well (NOTE: $0.5-1 \mathrm{~mL}$ of trypsin per well is sufficient)

5.4.4.6 Place well plates into the incubator. Check plates frequently under the microscope to ensure that all cells have lifted off. Timing will vary depending on the confluency. (NOTE: Do not leave the cells in trypsin for longer time than needed as it will damage the cells)

5.4.4.7 Once cells have completely lifted off the well plate, place back into the hood using proper aseptic techniques described above.

5.4.4.8 Follow steps 5.4.3.9-5.4.3.16.

Completed by (Initials/Date) Verified by (Initials/Date)

\subsection{Clean-up:}

5.5.1 Remove all materials out of the hood and replace in their appropriate locations.

5.5.2 Spray and wipe down the hood when completed. 
5.5.3 Discard of waste into the appropriate waste container.

5.6 Further feeding considerations

5.6.1 Changing of media should be done every 48-72 hours. Refer to the changing media SOP. 
Chapter 4

\section{THEORETICAL AND EXPERIMENTAL DETERMINATION OF NITRIC OXIDE CONSUMPTION BY RED BLOOD CELLS}




\subsection{Abstract}

A major determinant of $\mathrm{NO}$ bioavailability in the SMCs is the rate of NO consumption by the RBCs and determining any reaction limitations. To address this concern, we have measured the rate of NO consumption utilizing a NO gas source flowing above an RBC solution at a specified hematocrit (Hct). The methodology incorporated allows for investigation at physiological Hct. The consumption rate constant of NO by RBCs $\left(\mathrm{k}_{\mathrm{bl}}\right)$ was estimated both theoretically and experimentally as a function of surface area $(S)$, partition coefficient $(\lambda)$, volumetric gas flow $(\mathrm{Q})$ and NO diffusivity in the solution $\mathrm{D}_{\mathrm{NO}}$. We found that $\mathrm{k}_{\mathrm{bl}}$ in porcine $\mathrm{RBCs}$ at $25^{\circ} \mathrm{C}$ and $45 \% \mathrm{Hct}$ was $3500 \pm 700 \mathrm{~s}^{-1}$ with a membrane permeability $\left(\mathrm{P}_{\mathrm{m}}\right)$ of $1.5 \mathrm{~cm} \cdot \mathrm{s}^{-1}$. A nonlinear dependence of $\mathrm{k}_{\mathrm{bl}}$ on Hct was noted suggesting a predominant role for extracellular diffusion as the limiting factor for the reaction between NO and RBCs.

\subsection{Introduction}

Nitric Oxide (NO) is an important signal transduction molecule with estimated physiological concentrations to be in the nanomolar range. NO is known to have a short half-life in vivo, and can be degraded by a number of reactions. However, its first order reactions with superoxide and heme containing proteins such as hemoglobin $(\mathrm{Hb})$, will determine its fate in the vasculature [1].

NO is active in a number of physiological processes including the regulation of the vascular smooth muscle tone. In a typical blood vessel, NO produced by the ECs rapidly migrate towards the smooth muscle cells (SMCs) causing vessel relaxation. Simultaneously, NO diffuses inwards of the vessel and reacts with red blood cells (RBCs) 
containing high concentrations of NO scavengers such as oxy and deoxy $\mathrm{Hb}$. Due to high in vitro reactivity rate constants of $\mathrm{NO}$ with $\mathrm{Hb}$, it is uncertain as to how much of the endothelium derived NO reaches the smooth muscles to maintain physiologically relevant concentrations needed to induce vasodilation $[2,3]$.

Several theoretical and experimental studies have been made to address this paradox [4-8]. Initial theoretical models only considered transport of free NO and ignored preservation of NO bioactivity by other compounds made by NO such as dinitrogen trioxide $\left(\mathrm{N}_{2} \mathrm{O}_{3}\right)$, nitrite anions $\left(\mathrm{NO}_{2}^{-}\right)$and nitrosothiols (RSNO). Initial simulations showed that physiological concentrations of $\mathrm{Hb}$ caused rapid scavenging of $\mathrm{NO}$ and challenged the feasibility of NO being the endothelium derived relaxing factor (EDRF) [5]. Theoretical models included a cell free layer next to the vessel wall, indicating a reason how NO might be able to diffuse through the smooth muscle escaping scavenging by RBCs [9].

It is also proposed that encapsulated $\mathrm{Hb}$ within the membranes of $\mathrm{RBCs}$ can slow the reaction of $\mathrm{NO}$ and $\mathrm{Hb}$ as compared to $\mathrm{NO}$ reacting with free $\mathrm{Hb}$ due to a membrane barrier. The diffusion limitations across the RBC membrane were investigated as reasoning for $\mathrm{NO}$ to escape scavenging. In one study, $\mathrm{NO}$ was compared to $\mathrm{O}_{2}$, given they are both small diatomic molecules and have similar diffusivities across RBCs. It was observed there was a significant diffusion layer surrounding the RBC providing a resistance to $\mathrm{O}_{2}$ uptake. This diffusion layer comes into play because as $\mathrm{O}_{2}$ gets consumed by the $\mathrm{RBC}$, the region surrounding it rapidly depletes in $\mathrm{O}_{2}$ content faster than the rate of replenishment by diffusion or stirring $[9,10]$. Many believe that this could be what happens with respect to NO. 
Other groups have measured the consumption of NO in a suspension of RBCs. It was noted that the half-life of NO is inversely proportional to the concentration of RBCs, independent of oxyHb concentration in the $\mathrm{RBCs}$, and that the disappearance of $\mathrm{NO}$ is first order in terms of $\mathrm{NO}$ concentration and first order in terms of $\mathrm{RBC}$ concentration. It was concluded that extracellular diffusion resistance inhibits NO consumption by the RBCs [11]. However, it was considered that the resistance remains the same independent of hematocrit (Hct).

Competition experiments have also been utilized to examine limitations of NO uptake by the RBCs. The purpose of this test is to differentiate extracellular diffusion limitation from transmembrane or intracellular resistance. If extracellular diffusion resistance is negligible, then the measured effective reaction rate constant, $K_{R B C}$, would remain invariant of the Hct or the extracellular free $\mathrm{Hb}$ concentration. Previous results showed that the $\mathrm{K}_{\mathrm{RBC}}$ approaches a constant only when the Hct was greater than $10 \%$, suggesting that at higher Hct, the extracellular diffusion resistance is negligible [12]. However, even at higher Hct, it was observed that NO consumption by RBCs is still 5001000 times slower than that by $\mathrm{Hb}$. The conclusion was that this was due to intrinsic RBC factors such as transmembrane diffusion limitations or intracellular mechanisms. Subsequently, a model was designed that detailed and took into account internal, external and membrane diffusion limitations [13]. The model was fitted to experimental data to estimate membrane permeability, which was found to be 2000 times lower as compared to conventional values $[1,6]$. Intracellular diffusion was dismissed as no change in NO consumption was observed following a reduction in intracellular $\mathrm{Hb}$ concentration. Other competition experimental data indicate that the main resistance to NO uptake by RBCs is 
not transmembrane resistance; rather it is extracellular diffusion limitation in the unstirred layer surrounding each RBC [14]. Low membrane permeability was the reason for these observations [11]. Other groups utlized pretreated RBCs in their competition experiments $[12,15]$. NO uptake by RBCs increased and decreased upon alteration of the band 3 which binds to the cytoskeleton. It was noted that metHb and denatured $\mathrm{Hb}$ binding to the RBC membrane or cytoskeleton affected NO uptake by the RBCs. Moreover, such alterations in NO uptake by RBCs were found to correlate with the vasodilation of isolated blood vessels. It was then concluded that the RBC membrane and cytoskeleton associated NO inert proteins provide a barrier for NO diffusion into the $\mathrm{RBCs}[15]$.

Analysis of $\mathrm{Hb}$-vesicles (HbVs) with different particle sizes using stopped-flow spectrophotometry was also used in investigating NO uptake by RBCs [41]. In this approach, the notion that the lipid membrane affected NO uptake by the Hb vesicles was dismissed since values suggest that the apparent $\mathrm{NO}$ binding rate constant of $\mathrm{HbV}$ at low intracellular $\mathrm{Hb}$ concentration $(1 \mathrm{~g} / \mathrm{dl})$ was similar to that of molecular $\mathrm{Hb}\left(2.6 \times 10^{7} \mathrm{M}^{-1} \mathrm{~s}^{-}\right.$ 1) [16]. When the intracellular Hb concentrations were raised, it was observed that the NO binding rate constants fell $\left(0.9 \times 10^{7} \mathrm{M}^{-1} \mathrm{~s}^{-1}\right)$, which further decrease to $0.5 \times 10^{7} \mathrm{M}^{-1} \mathrm{~s}^{-1}$ when enlarging the particle diameter from 265 to $452 \mathrm{~nm}$. With the use of diffusion simulations and elementary binding reactions, it was concluded that an intracellular diffusion barrier is the predominant obstacle to $\mathrm{NO}$ binding with $\mathrm{Hb}$ [16]. In a later study, the effects of $\mathrm{HbVs}$ perfused through narrow gas permeable tubes were examined [17]. Four kinds of $\mathrm{Hb}$ containing solutions (purified $\mathrm{Hb}$, polymerized bovine $\mathrm{Hb}$ (Poly $\mathrm{Hb}), \mathrm{HbVs}$ [279 nm], and RBCs) were perfused through artificial narrow tubes of 
$25 \mu \mathrm{m}$ inner diameter at $\mathrm{Hb}$ concentration of $10 \mathrm{~g} / \mathrm{dl}$, at $1 \mathrm{~mm} \cdot \mathrm{s}^{-1}$. NO reactivity showed that $\mathrm{NO}$ binding with deoxygenated $\mathrm{Hb}$ and $\mathrm{Poly}_{\mathrm{B}} \mathrm{Hb}$ in the tube was faster than those with $\mathrm{HbV}$ and $\mathrm{RBCs}$, which showed almost identical binding rates. It was concluded that lateral diffusivity of $\mathrm{Hb} \& \mathrm{Poly}_{\mathrm{B}} \mathrm{Hb}$ lead to high consumption of $\mathrm{NO}$ as compared to $\mathrm{HbV}$ and RBCs [17].

Recent experimental work in a novel bioreactor tried to ascertain extracellular diffusion and permeability effects on NO and RBC interactions [18]. The bioreactor had then RBC solutions of $5 \%$ \& $45 \%$ Hct suspended in ice cold buffer and were kept constantly stirred. A headspace for the solutions was kept, which maintained controlled NO concentrations, along with the required inlets and outlets. Samples were collected at regular intervals and analyzed for NO oxidation derivatives. For a physiological Hct of $45 \%$, a reaction rate constant of $3.17 \times 10^{5} \mathrm{M}^{-1} \mathrm{~s}^{-1}$ was utilized, which is comparable to others groups $[11,19,20]$. However, this was obtained with a low $\mathrm{P}_{\mathrm{m}}$ of $0.0415-0.4$ $\mathrm{cm} \cdot \mathrm{s}^{-1}$, which suggests that $\mathrm{P}_{\mathrm{m}}$ is a critical factor prohibiting NO uptake by RBCs.

Mathematical models can also be implemented in examining NO reactivity with RBCs. One model was developed with the assumption of a spherical geometry for the RBC [20]. The RBC model included an intracellular region where $\mathrm{Hb}$ was encapsulated, a membrane region, and an extracellular plasma layer. The purpose of this model was to provide a means to study analytically extracellular, intracellular and membrane limited diffusion of NO into the RBCs. According to this model, NO had to cross both the extracellular region, and the membrane to enter the intracellular region where it could then bind with $\mathrm{Hb}$. This model successfully replicated results from competition experiments by taking a high membrane permeability $\left(\mathrm{P}_{\mathrm{m}}=40 \mathrm{~cm} \cdot \mathrm{s}^{-1}\right)$, and an oxy $\mathrm{Hb}$ 
binding constant at $25^{0} \mathrm{C}\left(\mathrm{K}_{\mathrm{oxyhb}}=106 \mu \mathrm{M}^{-1} \mathrm{~s}^{-1}\right)$ [13]. Simulations showed, at their chosen $\mathrm{P}_{\mathrm{m}}$, membrane resistance had little contribution. The major resistance to NO uptake was through extracellular diffusion. The reported NO consumption rate was $6.5 \times 10^{3} \mathrm{~s}^{-1}$ on a per blood volume basis of $0.7 \mu \mathrm{M}^{-1} \mathrm{~s}^{-1}$ and on a per total heme concentration basis [20].

On the whole, parameter values as well as a final conclusion regarding what actually prevents NO uptake by the RBCs has yet to be elucidated. This information would be essential in understanding more about NOs role as a vasodilator. Moreover, this information would be particularly useful in designing extracellular Hb-based oxygen carriers (HbOCs) which may be used as an alternative for blood transfusion. The benefits of such carriers are abundant, since the absence of antigens can help compatibility across different blood groups. In addition, purely artificially manufactured HbOCs can ensure good quality as well as decrease the transfection of diseases. However, the hypertensive effects seen after the administration of HBOCs preclude their more common usage [21, $22,23,24,25,26,27]$. Improvements over this are the $\mathrm{HbVs}$ or liposome encapsulated $\mathrm{Hb}[16]$.

We have attempted to study NO consumption by RBCs by first experimentally estimating NO consumption rates by RBCs at physiological Hct. Measurements obtained are of consumption of gaseous $\mathrm{NO}$ flowing over a buffer with a known amount of RBCs flowing through a chamber. In addition to obtaining a consumption rate, experimental work would give insight into the limiting factor of NO consumption by RBCs. These results have been verified against a previously established mathematical model [20]. 


\subsection{Methods}

Flow chamber design. Prior to experimentation, mathematical modeling was done to provide the design parameters of the chamber. Three important characteristics were considered in the model: convection, diffusion, and the reaction rate of NO with RBCs. Analytical and finite element methods (FEM) were used to model the system.

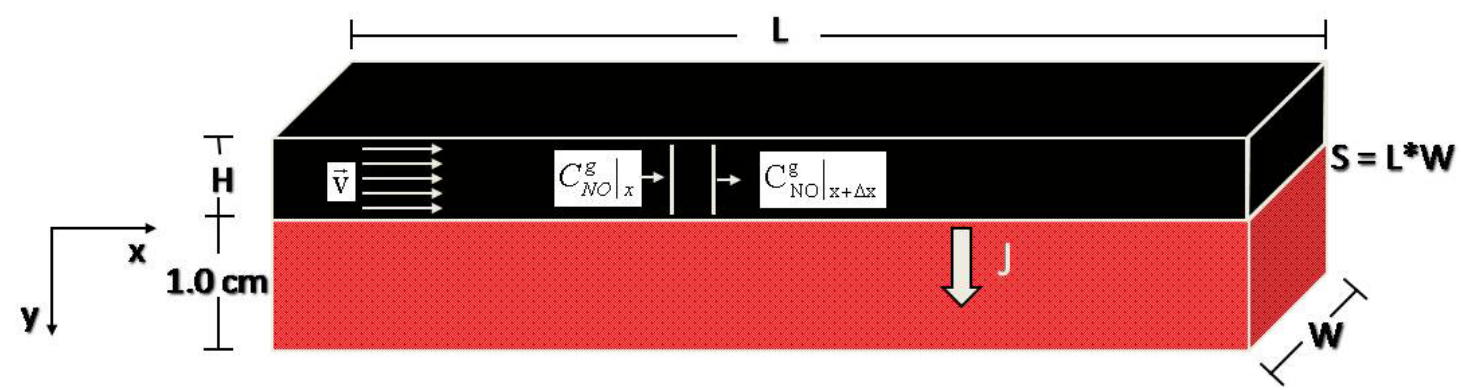

Figure 4.1 - Flow chamber schematic.

Analytical method. As shown in Figure 4.1, the flow chamber has several characteristics that play an important role in the analysis. In the upper part of the chamber there is convection of NO gas at a specific velocity $(v)$. When the NO gas comes in contact with the RBC solution below it diffuses at a flux (J). The diffusion area (S) is determined by the length (L) and width (W). The height $(\mathrm{H})$ corresponds to the height of the gaseous phase. It is important to utilize a low value for $\mathrm{H}$ in the chamber design to minimize gas phase resistance in NO transport. The assumptions used for the analytical approach were: plug flow in the gas region, negligible gas phase resistance, homogenous Hct in the buffer solution, and abundance of $\mathrm{Hb}$. Equation 3.1 shows the mass balance equations used. 


$$
\mathrm{A} \cdot \Delta \mathrm{x}\left(\frac{\mathrm{dC} \mathrm{NO}_{\mathrm{NO}}^{\mathrm{g}}}{\mathrm{dt}}\right)=\left.\mathrm{V}_{\mathrm{NO}} \cdot \mathrm{C}\right|_{\mathrm{x}}-\left.\mathrm{V}_{\mathrm{NO}} \cdot \mathrm{C}\right|_{\mathrm{x}+\Delta \mathrm{x}}-\mathrm{J} \cdot \Delta \mathrm{x} \cdot \mathrm{W}
$$

FEM analysis. FEM analysis was performed using FEMLAB-COMSOL, a modeling software allowing the simulation of physical processes defined by partial differential equations. FEM was used to predict how NO concentrations varied in the flow chamber and to test model assumptions derived from the analytical solution. A 2-D experimental chamber was defined in the software with the specifications obtained from the analytical solution. 2-D equations were used in the model to describe convection, diffusion and reaction. The assumption for the FEMLAB simulations was homogenous Hct.

Flow chamber preparation and data acquisition. Figure 4.2 shows a schematic of the experimental flow chamber design used in this study. The chamber allows for gaseous NO to flow through the top of the chamber, allowing direct contact with the RBC solution with a known Hct flowing at the bottom. The gaseous NO has a concentration of $45 \mathrm{ppm}$ and was supplied through one of the sides of the chamber at a constant and controlled volumetric flow rate. Once the NO enters the chamber, it reacts with the RBCs via diffusion. The pressure inside the chamber is kept at atmospheric pressure allowing flow from one side to the other without significant resistance. The concentration of the $\mathrm{NO}$ gas is measured at the exit of the flow chamber using a Nitric Oxide Analyzer (NOA) (GE; Boulder, CO). This instrument is a highly sensitive detector for measuring NO. This system allows measurement of NO consumption by $\mathrm{RBC}$ at high and physiological Hct. The NOA is connected to a computer allowing 
collection and processing of data acquired. For each experiment, different Hct were prepared $(15 \%, 30 \%, 40 \%, 45 \%, 50 \%, 55 \%$, and $60 \%)$. The exiting NO gas concentration was examined for each run.

In addition, the RBC solution flowing at the bottom of the flow chamber was analyzed to ensure that the solution maintained laminar flow. Calculations of the Reynolds number in both the tubing of the system as well as the flow chamber itself were performed. The Reynolds numbers were found to be 157 and 24.5 for the tubing and chamber respectively. It was noted that both of these values were under 2100 therefore ensuring that the solution flowing through the system was laminar.

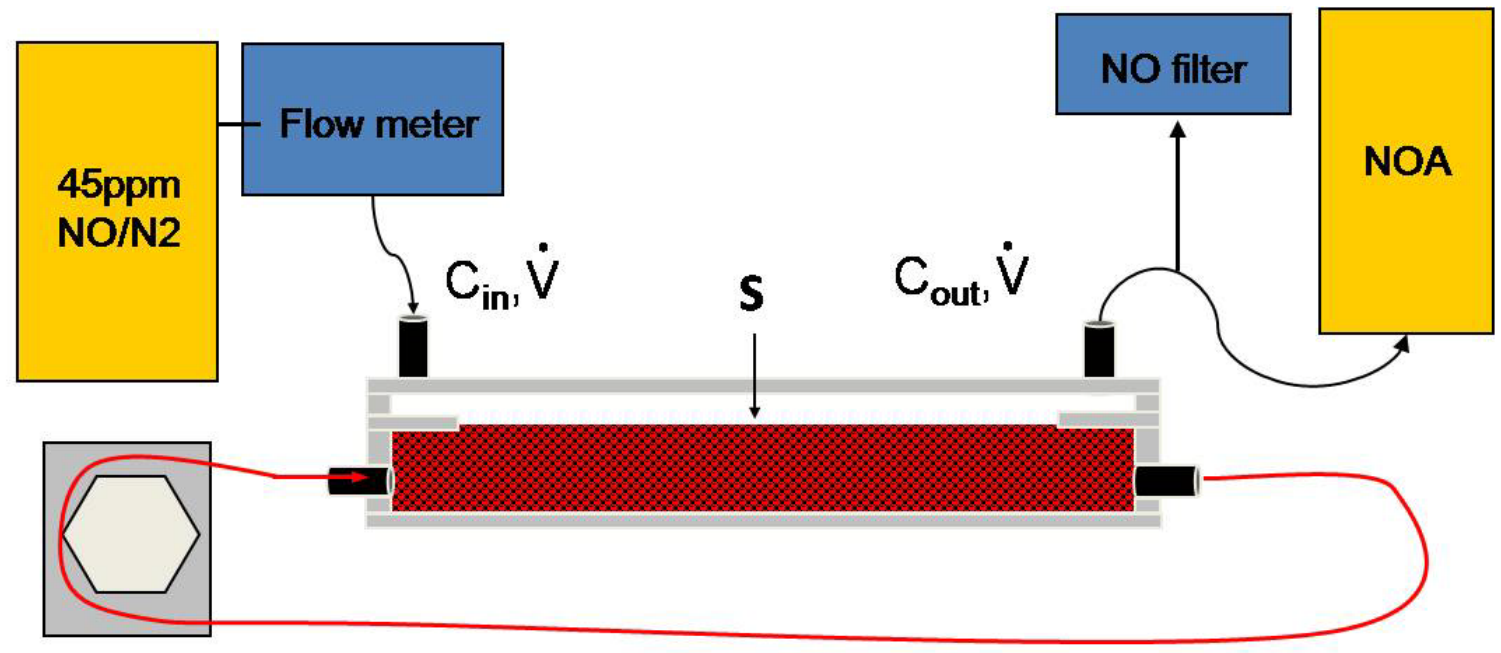

\section{Peristaltic} pump

Figure 4.2 - Schematic of experimental flow chamber design.

Blood collection and separation. Experimental studies incorporated the use of porcine blood. $500 \mathrm{~mL}$ of blood was collected in a container with $50 \mathrm{~mL}$ of heparin (Sigma-Aldrich; St. Louis, MO) in a buffer solution. The sample was then transferred to 
$50 \mathrm{~mL}$ conical tubes and centrifuged at $800 \mathrm{~g}$ for 10 minutes. The supernatant containing the plasma and buffy coat were removed. The remaining RBCs were resuspended and washed three times with a buffer solution containing $20 \mathrm{mM}$ Hepes (Sigma-Aldrich; St. Louis, MO), $140 \mathrm{mM} \mathrm{NaCl}$ (Sigma-Aldrich; St. Louis, MO), and $5 \mathrm{mM}$ glucose (Invitrogen; Carlsbad, CA) with a pH of 7.4 and 295 mOsm. After each wash, the cells were centrifuged at the aforementioned condition. The remaining sample was diluted with buffer solution to obtain the desired Hct and maintained at $25^{\circ} \mathrm{C}$.

$\mathrm{Hb}$ measurement. Before and after each experimental run, $10 \mathrm{~mL}$ of blood was separated and centrifuged for $15 \mathrm{~min}$ at $800 \mathrm{~g}$. $1 \mathrm{~mL}$ of the plasma was removed and analyzed for free $\mathrm{Hb}$ using a spectrophotometer. This measurement is important to ensure that $\mathrm{NO}$ consumption is by $\mathrm{RBCs}$ and not by free $\mathrm{Hb}$. The spectrophotometer measures concentrations of metHb, deoxyHb and oxyHb based on absorbances at their respective wavelengths [15].

\subsection{Results}

FEM model. The chamber defined in FEMLAB required implementing a very fine mesh close to the gas-liquid interface to be able to capture the steep gradient in the $\mathrm{NO}$ concentration profile as most of the NO is consumed in the first few $\mu \mathrm{m}$ of the liquid phase. According to the FEMLAB results, once NO is in contact with the RBC solution, it only has to travel several microns before the NO starts to react. FEMLAB was also utilized to validate the equations derived from the analytical method. Graphical solutions from both analyzing methods were superimposed. The graph from the analytical method was obtained by creating a continuous graph that showed the ratio of $\mathrm{C}_{\text {out }} / \mathrm{C}_{\text {in }}$ with respect 
to NO reaction rates with the $\mathrm{RBCs}\left(\mathrm{k}_{\mathrm{bl}} \mathrm{s}^{-1}\right)$ by increasing the value of $\mathrm{k}_{\mathrm{bl}}$ from 500-6000 using Equation 3.2.

$$
C_{\text {out }}=C_{\text {in }} \cdot e^{\frac{-\lambda \cdot S}{V_{\mathrm{No}}} \sqrt{\left(k_{b l} \cdot D\right)}}
$$

The graphical image from FEMLAB was obtained by performing one simulation at a time similarly by increasing $\mathrm{k}_{\mathrm{bl}}$ from $500-6000$. Figure 4.3 displays the superimposed image for both ratio graphs. Both models showed very similar trends suggesting experimental analysis should give an approximation to these theoretical yields.

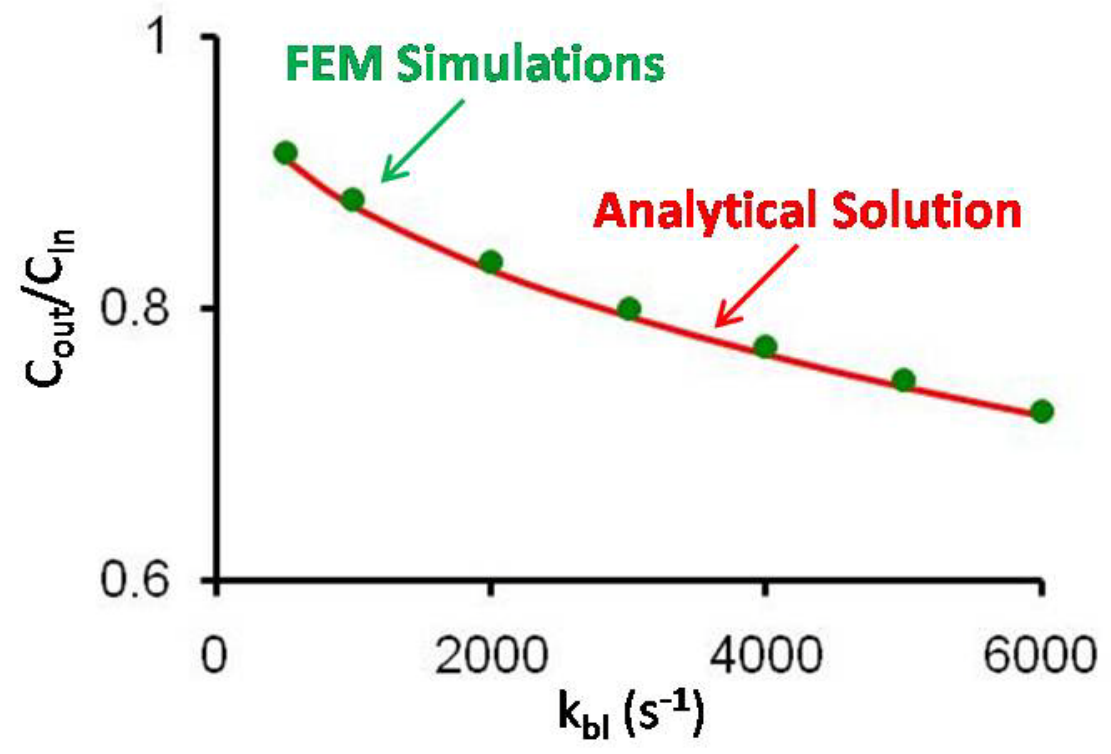

Figure 4.3 - FEMLAB and analytical solution comparison.

Experimental results. Experimental results were compared to a previously developed mathematical model which also predicted $\mathrm{k}_{\mathrm{bl}}$ as a function of Hct [20]. The model, however, differed to the experimental conditions. The model was developed to predict values at $37{ }^{\circ} \mathrm{C}$ and extracellular $\mathrm{Hb}$ was not taken into consideration. Experimental conditions were actually performed at $25{ }^{\circ} \mathrm{C}$ and extracellular $\mathrm{Hb}$ was 
accounted for, therefore the model was modified to incorporate these changes. The change in temperature conditions changed the diffusivity constant of NO in the buffer solution from $3.3 \times 10^{-5} \mathrm{~cm}^{2} \mathrm{~s}^{-1}$ at $37^{\circ} \mathrm{C}$ to $2.21 \times 10^{-5} \mathrm{~cm}^{2} \mathrm{~s}^{-1}$ at $25^{\circ} \mathrm{C}$ [28]. Extracellular $\mathrm{Hb}$ was taken into consideration by evaluating how much was present before and after each experimental run. It was noted that at $60 \% \mathrm{Hct}$, the highest Hct tested, extracellular $\mathrm{Hb}$ was found to be $35 \pm 5 \mu \mathrm{M}$. In order to account for extracellular $\mathrm{Hb}$ at each Hct solution, Equation 3.3 was assumed.

$$
\mathrm{C}_{\mathrm{Hb}, \text { plasma }}=\frac{\mathrm{Hct}}{(1-\mathrm{Hct})} \cdot 25 \mu 5
$$

Another factor that had to be modified was the reaction rate between NO and oxyHb. The previous model used a $\mathrm{k}_{\text {oxy }}$ of $80 \mu \mathrm{M}^{-1} \mathrm{~s}^{-1}$, while experimental data performed with the flow chamber exhibited different values [20]. The same procedure used to measure the reaction rate of $\mathrm{NO}$ with RBCs within the flow chamber was utilized to determine $\mathrm{k}_{\text {oxy }}$ using oxyHb in buffer. According to experimental data, $\mathrm{k}_{\mathrm{oxy}}$ was calculated to be approximately $25 \mu \mathrm{M}^{-1} \mathrm{~s}^{-1}$. Although this value is lower than previously reported values, this value was used in the model in order to maintain the same conditions for the measurements of NO uptake by RBCs. 


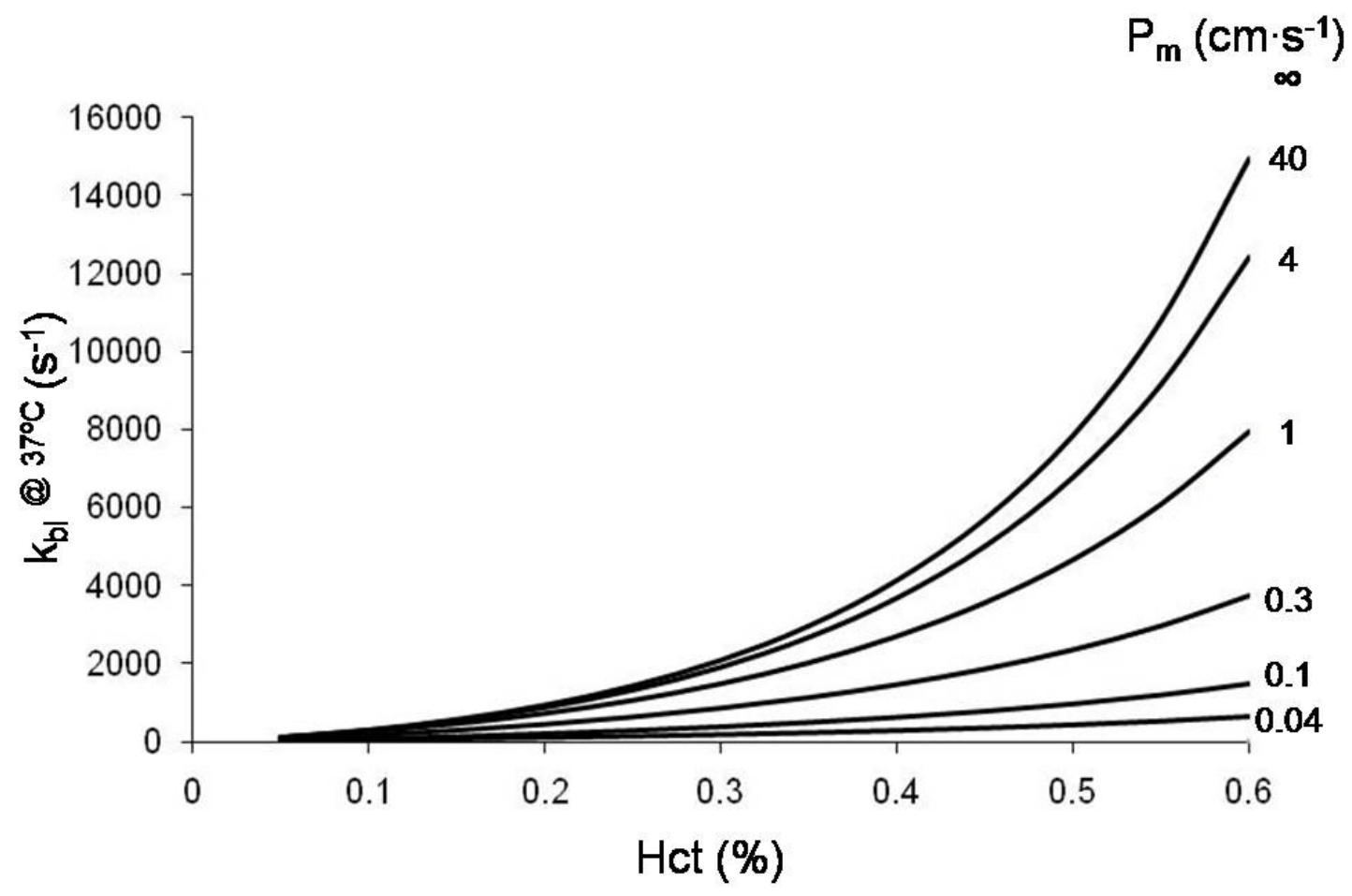

Figure 4.4 - Previous model predictions for the observed first order reaction rate constant of $\mathrm{NO}$ in blood $\left(37^{\circ} \mathrm{C}, 45 \% \mathrm{Hct}\right), \mathrm{k}_{\mathrm{bl}}[20]$

Figure 4.4 exhibits the previous model's predictions on how $\mathrm{k}_{\mathrm{bl}}$ behaves with respect to Hct and $\mathrm{P}_{\mathrm{m}}$. At $45 \%$ Hct, predictions for $\mathrm{k}_{\mathrm{bl}}$ vary between $7.5 \times 10^{2}$ and $6.5 \times 10^{3}$ $\mathrm{s}^{-1}$ when $\mathrm{P}_{\mathrm{m}}$ changes between 0.04 and $40 \mathrm{~cm} \cdot \mathrm{s}^{-1}$ [20]. Figure 4.5 however, shows this same relationship incorporating the different temperature and $\mathrm{k}_{\mathrm{oxy}}$ values, inclusion of extracellular $\mathrm{Hb}$, as well as experimental data observed from flow chamber experiments. 


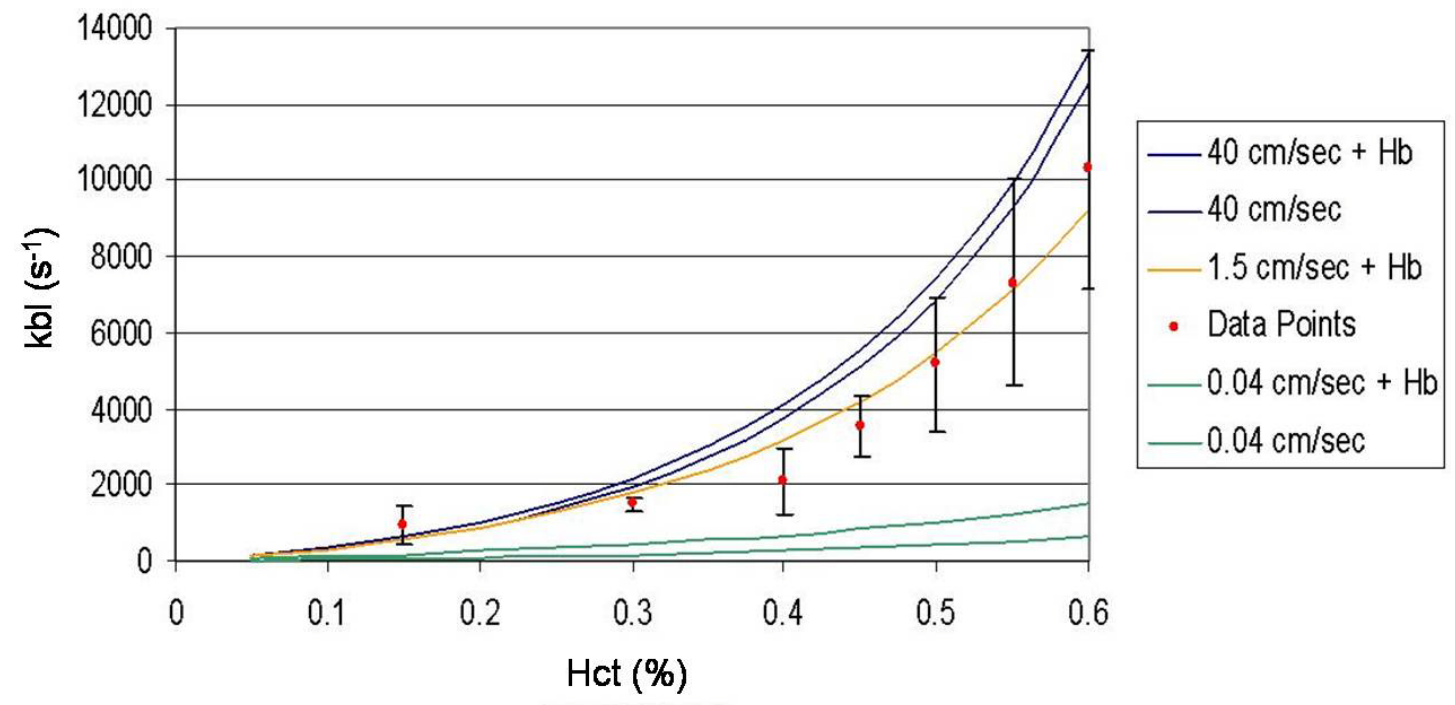

Figure 4.5 - Updated model predictions of $\mathrm{k}_{\mathrm{bl}}$ at $25^{\circ} \mathrm{C}$ including experimental data.

According to the experimental observations, at low Hct it is not distinguishable whether the values follow the low or high $\mathrm{P}_{\mathrm{m}}$ values. However, once the Hct reaches physiological levels, it is noted that the extracellular space between the RBCs diminishes and allows the $\mathrm{NO}$ to diffuse faster to the $\mathrm{RBCs}$, increasing the reaction rate. A trendline was added by minimizing the SSE of the experimental values. This fitting suggests an experimental $\mathrm{P}_{\mathrm{m}}$ value of approximately $1.5 \mathrm{~cm} \cdot \mathrm{s}^{-1}$ and a reaction rate constant $\mathrm{k}_{\mathrm{bl}}$ of $3500 \pm 700 \mathrm{~s}^{-1}$.

\subsection{Discussion}

The estimated $\mathrm{k}_{\mathrm{bl}}$ for porcine $\mathrm{RBCs}$ at $25^{\circ} \mathrm{C}$ and $45 \%$ Hct was found to be approximately $3500 \mathrm{~s}^{-1}$. In addition, the calculated $\mathrm{P}_{\mathrm{m}}$ was found to be $1.5 \mathrm{~cm} / \mathrm{s}$. It has been previously discussed that the actual mechanism of how NO is able to escape being scavenged by RBCs has yet to be established. Therefore, the value of $\mathrm{P}_{\mathrm{m}}$ was determined in order to differentiate whether NO consumption is limited by extracellular diffusion or 
membrane resistance. In this study, extracellular diffusion was shown to play a predominant role in the limiting of NO consumption due to several factors. As previously mentioned, if extracellular diffusion resistance is negligible and would ultimately not play a role in limiting NO uptake, then the effective reaction rate constant, $\mathrm{k}_{\mathrm{bl}}$, would remain invariant of the Hct values. However, our experimental data clearly exhibits an apparent nonlinear dependence of $\mathrm{k}_{\mathrm{bl}}$ and Hct, therefore suggesting the conclusion that extracellular diffusion is indeed the reason for limiting NO uptake by RBCs.

In addition, $\mathrm{P}_{\mathrm{m}}$ values that vary between $1-40 \mathrm{~cm} \cdot \mathrm{s}^{-1}$ also exhibit this nonlinear behavior. Our experimental data yielded a value of $1.5 \mathrm{~cm} \cdot \mathrm{s}^{-1}$ which falls in this range and further corroborates our conclusion. Concluding that extracellular diffusion as the limiting mechanism also aids in explaining how NO's reaction rate with RBCs is similar to oxygen's reaction rate with $\mathrm{Hb}$ which is noted to be limited by extracellular diffusion and not by RBCs membrane [10]. However, it was noted that $\mathrm{P}_{\mathrm{m}}$ values smaller than 1 $\mathrm{cm} \cdot \mathrm{s}^{-1}$ exhibited a somewhat linear relationship. This type of behavior on the other hand suggests membrane resistance as the limiting factor rather than extracellular diffusion. Therefore, the value of $\mathrm{P}_{\mathrm{m}}$ is a critical variable needed to determine the actual limiting factor of NO uptake by RBCs.

Summary. This study was successful in measuring NO consumption by RBCs to predict both the reaction rates $\left(\mathrm{k}_{\mathrm{bl}}\right)$ and $\mathrm{P}_{\mathrm{m}}$ value at physiological Hct. With these information, we were also able to distinguish a means of how NO is able to escape scavenging by the RBCs. Prior experimental studies measuring NO consumption above $20 \%$ Hct had not been properly performed. The experimental setup for these groups 
measured NO consumption at low Hct incorporating the use of NO donors and NO sensitive electrodes with measurements taken after different time intervals. Using this prior technique, measurements at higher or physiological Hct could not be possible. This is because the reaction between $\mathrm{NO}$ with RBCs occurs within milliseconds, therefore measurements in time intervals would not capture the reaction. A proper measuring technique at physiological Hct must be able to capture real-time information.

The experimental model incorporated in this study differs from previous studies in that $\mathrm{NO}$ gas is the source of NO used as opposed to NO donors. This method is advantageous in that the NO consumed within the system is limited only by the surface area of the liquid-gas interface. In addition, there is a reduced NO concentration dissolved in the solution due to the low solubility of NO $(\lambda=0.041$ mol $\left.\left(\mathrm{NO}_{\text {liq }}\right) / \mathrm{mol}\left(\mathrm{NO}_{\mathrm{gas}}\right)\right)$. These advantages allowed measurements at physiological Hct possible. These newly determined values for $\mathrm{NO}$ consumption and $\mathrm{P}_{\mathrm{m}}$ can be further combined with derived NO production rates to yield information about the NO paradox and bioavailability of NO within the SMCs.

\subsection{Acknowledgements}

This work was funded by National Institutes of Health Grant SC1HL095101 (NT).

\subsection{References}

[1] J.S. Beckman, and W.H. Koppenol, Nitric oxide, superoxide, and peroxynitrite: the good, the bad, and ugly. Am J Physiol 271 (1996) C1424-37.

[2] R.F. Eich, T. Li, D.D. Lemon, D.H. Doherty, S.R. Curry, J.F. Aitken, A.J. Mathews, K.A. Johnson, R.D. Smith, G.N. Phillips, Jr., and J.S. Olson, Mechanism of NO-induced oxidation of myoglobin and hemoglobin. Biochemistry 35 (1996) 6976-83. 
[3] S. Herold, M. Exner, and T. Nauser, Kinetic and mechanistic studies of the NO*mediated oxidation of oxymyoglobin and oxyhemoglobin. Biochemistry 40 (2001) 3385-95.

[4] A.R. Butler, I.L. Megson, and P.G. Wright, Diffusion of nitric oxide and scavenging by blood in the vasculature. Biochim Biophys Acta 1425 (1998) 16876.

[5] J.R. Lancaster, Jr., Simulation of the diffusion and reaction of endogenously produced nitric oxide. Proc Natl Acad Sci U S A 91 (1994) 8137-41.

[6] T. Malinski, Z. Taha, S. Grunfeld, S. Patton, M. Kapturczak, and P. Tomboulian, Diffusion of nitric oxide in the aorta wall monitored in situ by porphyrinic microsensors. Biochem Biophys Res Commun 193 (1993) 1076-82.

[7] M.W. Vaughn, K.T. Huang, L. Kuo, and J.C. Liao, Erythrocyte consumption of nitric oxide: competition experiment and model analysis. Nitric Oxide 5 (2001) $18-31$.

[8] J. Wood, and J. Garthwaite, Models of the diffusional spread of nitric oxide: implications for neural nitric oxide signalling and its pharmacological properties. Neuropharmacology 33 (1994) 1235-44.

[9] J.T. Coin, and J.S. Olson, The rate of oxygen uptake by human red blood cells. J Biol Chem 254 (1979) 1178-90.

[10] V.H. Huxley, and H. Kutchai, The effect of the red cell membrane and a diffusion boundary layer on the rate of oxygen uptake by human erythrocytes. J Physiol 316 (1981) 75-83.

[11] X. Liu, M.J. Miller, M.S. Joshi, H. Sadowska-Krowicka, D.A. Clark, and J.R. Lancaster, Jr., Diffusion-limited reaction of free nitric oxide with erythrocytes. J Biol Chem 273 (1998) 18709-13.

[12] M.W. Vaughn, L. Kuo, and J.C. Liao, Effective diffusion distance of nitric oxide in the microcirculation. Am J Physiol 274 (1998) H1705-14.

[13] M.W. Vaughn, K.T. Huang, L. Kuo, and J.C. Liao, Erythrocytes possess an intrinsic barrier to nitric oxide consumption. J Biol Chem 275 (2000) 2342-8.

[14] X. Liu, A. Samouilov, J.R. Lancaster, Jr., and J.L. Zweier, Nitric oxide uptake by erythrocytes is primarily limited by extracellular diffusion not membrane resistance. J Biol Chem 277 (2002) 26194-9. 
[15] K.T. Huang, T.H. Han, D.R. Hyduke, M.W. Vaughn, H. Van Herle, T.W. Hein, C. Zhang, L. Kuo, and J.C. Liao, Modulation of nitric oxide bioavailability by erythrocytes. Proc Natl Acad Sci U S A 98 (2001) 11771-6.

[16] H. Sakai, N. Okuda, A. Sato, T. Yamaue, S. Takeoka, and E. Tsuchida, Hemoglobin encapsulation in vesicles retards $\mathrm{NO}$ and $\mathrm{CO}$ binding and $\mathrm{O} 2$ release when perfused through narrow gas-permeable tubes. Am J Physiol Heart Circ Physiol 298 (2010) H956-65.

[17] H. Sakai, A. Sato, K. Masuda, S. Takeoka, and E. Tsuchida, Encapsulation of concentrated hemoglobin solution in phospholipid vesicles retards the reaction with NO, but not CO, by intracellular diffusion barrier. J Biol Chem 283 (2008) 1508-17.

[18] P. Deonikar, and M. Kavdia, Extracellular diffusion and permeability effects on NO-RBCs interactions using an experimental and theoretical model. Microvasc Res 79 (2010) 47-55.

[19] E. Carlsen, and J.H. Comroe, Jr., The rate of uptake of carbon monoxide and of nitric oxide by normal human erythrocytes and experimentally produced spherocytes. J Gen Physiol 42 (1958) 83-107.

[20] N.M. Tsoukias, and A.S. Popel, Erythrocyte consumption of nitric oxide in presence and absence of plasma-based hemoglobin. Am J Physiol Heart Circ Physiol 282 (2002) H2265-77.

[21] A.I. Alayash, Hemoglobin-based blood substitutes: oxygen carriers, pressor agents, or oxidants? Nat Biotechnol 17 (1999) 545-9.

[22] C.P. Stowell, J. Levin, B.D. Spiess, and R.M. Winslow, Progress in the development of RBC substitutes. Transfusion 41 (2001) 287-99.

[23] J.A. Ulatowski, Y. Asano, R.C. Koehler, R.J. Traystman, and E. Bucci, Sustained endothelial dependent dilation in pial arterioles after crosslinked hemoglobin transfusion. Artif Cells Blood Substit Immobil Biotechnol 25 (1997) 115-20.

[24] J.A. Ulatowski, R.C. Koehler, T. Nishikawa, R.J. Traystman, A. Razynska, H. Kwansa, B. Urbaitis, and E. Bucci, Role of nitric oxide scavenging in peripheral vasoconstrictor response to beta beta cross-linked hemoglobin. Artif Cells Blood Substit Immobil Biotechnol 23 (1995) 263-9.

[25] J.A. Ulatowski, T. Nishikawa, B. Matheson-Urbaitis, E. Bucci, R.J. Traystman, and R.C. Koehler, Regional blood flow alterations after bovine fumaryl beta betacrosslinked hemoglobin transfusion and nitric oxide synthase inhibition. Crit Care Med 24 (1996) 558-65. 
[26] R.M. Winslow, Blood substitutes: refocusing an elusive goal. Br J Haematol 111 (2000) 387-96.

[27] R.M. Winslow, alphaalpha-crosslinked hemoglobin: was failure predicted by preclinical testing? Vox Sang 79 (2000) 1-20.

[28] I.G. Zacharia, and W.M. Deen, Diffusivity and solubility of nitric oxide in water and saline. Ann Biomed Eng 33 (2005) 214-22. 


\section{Appendix 4.1}

Mass balance of the gaseous NO through the chamber yields:

$$
\begin{aligned}
& \mathrm{A} \cdot \Delta \mathrm{x}\left(\frac{\mathrm{dC}_{\mathrm{NO}}^{\mathrm{g}}}{\mathrm{dt}}\right)=\mathrm{V}_{\mathrm{NO}} \cdot \mathrm{C}_{\mathrm{x}}-\left.\mathrm{V}_{\mathrm{NO}} \cdot \mathrm{C}\right|_{\mathrm{x}+\Delta \mathrm{x}}-\mathrm{J} \cdot \Delta \mathrm{x} \cdot \mathrm{W} \\
& \left(\frac{\mathrm{dC}_{\mathrm{NO}}^{\mathrm{g}}}{\mathrm{dt}}\right)=\overrightarrow{\mathrm{v}}\left(-\frac{\mathrm{dC}^{\mathrm{g}}}{\mathrm{dx}}\right)-\frac{\mathrm{J}}{\mathrm{L}}
\end{aligned}
$$

At steady state:

$$
0=\frac{\mathrm{V}_{\mathrm{NO}}}{\mathrm{A}}\left(-\frac{\mathrm{dC}}{\mathrm{dx}}\right)-\frac{\mathrm{J} \cdot \mathrm{W}}{\mathrm{A}}
$$

where $\mathrm{C}_{\mathrm{NO}}$ is the $\mathrm{NO}$ concentration, $\mathrm{V}_{\mathrm{NO}}$ is the volumetric flow, $\mathrm{J}$ is the flux of $\mathrm{NO}$ across the RBC solution-gas interface, $\mathrm{A}$ is the gas phase cross-sectional area, $\mathrm{W}$ is the width, and $\mathrm{L}$ is the length.

Diffusion of NO gas into the RBC buffer solution with known Hct yields:

$$
\begin{aligned}
& \mathrm{A}_{1} \cdot \Delta \mathrm{y}\left(\frac{\mathrm{dC}_{\mathrm{NO}}^{1}}{\mathrm{dt}}\right)=-\left.\mathrm{D} \cdot \frac{\mathrm{dC}}{\mathrm{dt}}\right|_{\mathrm{y}}+\left.\mathrm{D} \cdot \frac{\mathrm{dC}}{\mathrm{dy}}\right|_{\mathrm{y}+\Delta \mathrm{y}}-\mathrm{C}^{1} \cdot \mathrm{k}_{\mathrm{bl}} \cdot \Delta \mathrm{A}_{1} \cdot \Delta \mathrm{y} \\
& \left(\frac{\mathrm{dC}_{\mathrm{NO}}^{1}}{\mathrm{dt}}\right)=\mathrm{D}_{\mathrm{NO}} \cdot \frac{\mathrm{d}^{2} \mathrm{C}^{1}}{\mathrm{dy}^{2}}-\mathrm{C}_{\mathrm{NO}}^{1} \cdot \mathrm{k}_{\mathrm{bl}}
\end{aligned}
$$

At steady state:

$$
0=\mathrm{D} \cdot \frac{\mathrm{d}^{2} \mathrm{C}}{\mathrm{dy}^{2}}-\mathrm{C}^{1} \cdot \mathrm{k}_{\mathrm{bl}}
$$

(Parameters: $\mathrm{D}$ is the diffusivity constant and $\mathrm{k}_{\mathrm{bl}}$ as the NO consumption rate)

Solving the steady state Eq. A.4.2: 


$$
\begin{aligned}
& C_{\text {out }}=C_{\text {in }} \cdot e^{\frac{-\lambda \cdot S}{V_{\mathrm{No}}} \sqrt{\left(\mathrm{k}_{\mathrm{bl}} \cdot \mathrm{D}\right)}} \\
& \mathrm{C}_{\text {out }}=\mathrm{C}_{\text {in }} \cdot \mathrm{e}^{\frac{-\lambda \cdot \mathrm{L}}{\mathrm{v} \cdot \mathrm{H}} \sqrt{\left(\mathrm{k}_{\mathrm{bl}} \cdot \mathrm{D}\right)}} \\
& \mathrm{k}_{\mathrm{bl}}=\frac{\left(\frac{\mathrm{v} \cdot \mathrm{H}}{\lambda \cdot \mathrm{L}} \cdot \ln \left(\frac{\mathrm{C}_{\text {in }}}{\mathrm{C}_{\text {out }}}\right)\right)^{2}}{\mathrm{D}}
\end{aligned}
$$

By using Eq. A.4.4, a value for $\mathrm{k}_{\mathrm{bl}}$ was calculated using the initial $[\mathrm{NO}]\left(\mathrm{C}_{\mathrm{in}}\right)$ and the output NO measured by the NOA $\left(\mathrm{C}_{\text {out }}\right)$. These values were used to predict $\mathrm{P}_{\mathrm{m}}[20]$. Eq. A.4.3 was utilized to perform preliminary simulations to predict the outflow concentration of NO for a $\mathrm{k}_{\mathrm{bl}}$ value of $6000 \mathrm{~s}^{-1}$ [19].

\begin{tabular}{|l|l|l|l|}
\hline Parameter & Value & Units & Description \\
\hline Hct & $15 \%-60 \%$ & - & Hematocrit \\
\hline D $_{\mathrm{NO}}$ & $2.21 \times 10^{-5}$ & $\mathrm{~cm}^{2} \mathrm{~s}^{-1}$ & Diffusivity constant of NO in RBC solution \\
\hline $\mathrm{S}$ & 100 & $\mathrm{~cm}^{2}$ & Area exposed to NO gas \\
\hline $\mathrm{W}$ & 2.5 & $\mathrm{~cm}$ & Chamber width \\
\hline $\mathrm{L}$ & 40 & $\mathrm{~cm}$ & Chamber length \\
\hline $\mathrm{H}$ & 0.5 & $\mathrm{~cm}$ & Gas phase height \\
\hline$v$ & 2 & $\mathrm{~cm} / \mathrm{s}$ & NO velocity \\
\hline$\lambda$ & 0.04 & - & Partition coefficient \\
\hline $\mathrm{V}$ & 0.3 & $\mathrm{~mL} / \mathrm{min}$ & NO volumetric flow rate \\
\hline
\end{tabular}

Table A.4.1 - Chamber parameters and values 
Chapter 5

SUMMARY 
Nitric oxide (NO) has been recognized for its importance in many physiological arenas including playing a major role in the regulation of vascular tone. Upon realization that endothelial derived NO is responsible for inducing vasodilation in the smooth muscle cells (SMCs) of the vasculature, many have studied its production and consumption properties. However, the phenomena known as the "NO paradox," questions NO's role as a vasodilator. Due to NO's known fast reaction with heme containing proteins such as hemoglobin $(\mathrm{Hb})$, and given that red blood cells (RBCs) containing $\mathrm{Hb}$ are traveling within close proximity to the site of NO production, it is unclear how endothelial derived NO escapes scavenging by the RBCs to diffuse to the adjacent SMCs to induce vasodilation. To determine both NO bioavailability in the SMCs and NO levels needed to induce vasodilation, information is needed about NO production and consumption within the vasculature. The balance between these rates will determine NO's ability to cause vasodilation The work performed for this dissertation was aimed at understanding this balance.

In an attempt to address the questions arising from the NO paradox, theoretical and experimental studies were performed to yield information about NO production and NO consumption rates within the vasculature. Cell culture studies utilizing Human Umbilical Vein Endothelial Cells (HUVECs) and incorporating the use of a NO-sensitive fluorescent dye, 4-amino-5-methylamino-2',7'-difluorescein diacetate (DAF-FM da), were performed to determine NO production rates. Solution experiments, without the presence of cells, were initially performed for the calibration of the fluorescent dye. These experimental results were verified against a detailed mathematical model that 
analyzed the kinetic behavior between DAF-FM and NO. Once a calibration protocol for the fluorescent dye was developed for both solution and cellular experiments, production rates and NO concentration levels were determined upon agonist stimulation. It was determined that at maximum agonist stimulation, the $\mathrm{NO}$ production rate was found to be $0.418 \pm 0.18 \mathrm{pmol} / \mathrm{cm}^{2} \mathrm{~s}$. Assuming an endothelial layer thickness of $4 \mu \mathrm{m}$, this is equivalent to $1.05 \mu \mathrm{M} / \mathrm{s}$. This production rate value was noted to be within the same range as was previously observed by [1]. This group also utilized HUVECs and detected NO using chemiluminescence

The second major parameter in addressing the "NO paradox" is NO consumption by RBCs. To address this concern, theoretical and experimental studies were performed by measuring the rate of $\mathrm{NO}$ consumption utilizing a $\mathrm{NO}$ gas source flowing above a porcine RBC solution at a specified hematocrit (Hct). The methodology incorporated for this study allowed for investigation and measurements at physiological Hct. The consumption rate constant of $\mathrm{NO}$ by $\mathrm{RBCs}\left(\mathrm{k}_{\mathrm{bl}}\right)$ was estimated both theoretically and experimentally as a function of surface area (S), partition coefficient $(\lambda)$, volumetric gas flow $(\mathrm{Q})$ and $\mathrm{NO}$ diffusivity in the solution $\mathrm{D}_{\mathrm{NO}}$. It was found that $\mathrm{k}_{\mathrm{bl}}$ in porcine $\mathrm{RBCs}$ at $25{ }^{\circ} \mathrm{C}$ and $45 \%$ Hct was $3500 \pm 700 \mathrm{~s}^{-1}$ with a membrane permeability $\left(\mathrm{P}_{\mathrm{m}}\right)$ of $1.5 \mathrm{~cm} \cdot \mathrm{s}^{-1}$. A previous study showed a range of similar reaction rate values that were predicted for $\mathrm{P}_{\mathrm{m}}$ values between $0.4-40 \mathrm{~cm} \cdot \mathrm{s}^{-1}$ at physiological Hct [2]. A nonlinear dependence of $\mathrm{k}_{\mathrm{bl}}$ on Hct as well as a nonlinear relationship of $\mathrm{P}_{\mathrm{m}}$ was experimentally observed suggesting a predominant role for extracellular diffusion as the limiting factor for the reaction between $\mathrm{NO}$ and RBCs. 
Once both NO production and consumption rates were determined experimentally, a mathematical model was utilized to determine NO bioavailability in the SMCs. A previously developed arteriolar model was utilized to yield this information [3]. This theoretical model incorporated a series of concentric cylinders to mimic the anatomy of a vessel. RBCs, at chosen Hct, flow within the lumen of the vessel. A RBCfree layer was integrated into the model between the flowing blood and the inner layer of the vessel composed of endothelial cells (ECs). Adjacent to the ECs is a layer of SMCs. It is assumed NO is transported in the radial direction into either the lumen or SMC region. Axial and angular NO transport was neglected with the assumption that there is symmetry in NO production [3]. Upon the addition of the recently acquired experimental NO production and consumption rates, NO profiles were generated to yield $\mathrm{NO}$ concentration levels within the SMCs. Several NO production rates varying between 0.1 $-1.0 \mathrm{pmol} / \mathrm{cm}^{2} \mathrm{~s}$ were modeled while also varying the NO consumption rate between $2500-6500 \mathrm{~s}^{-1}$ at $45 \%$ Hct.

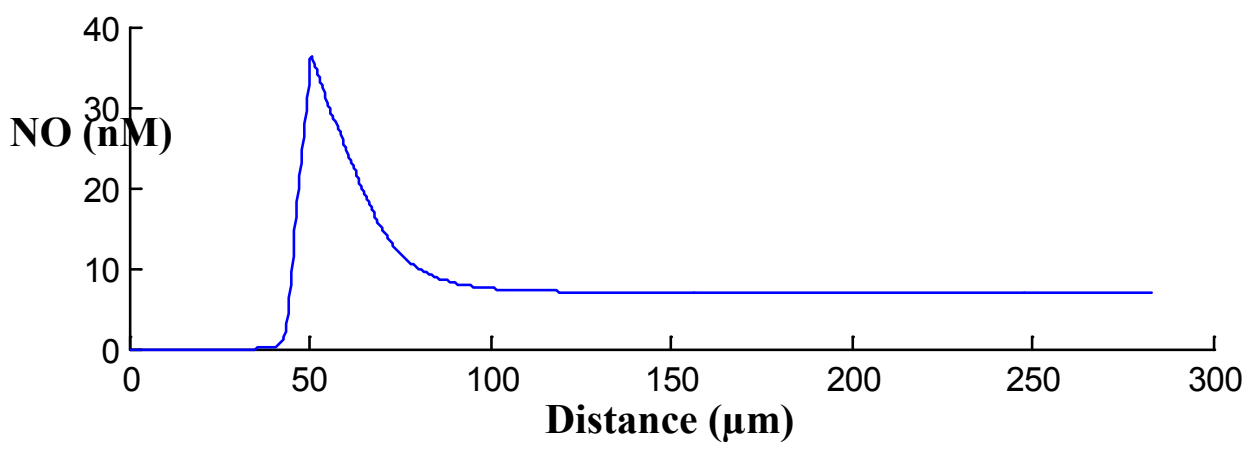

Figure 5.1 - NO concentration levels available in the SMCs assuming an endothelial derived NO production rate of $1.0 \mathrm{pmol} / \mathrm{cm}^{2} \mathrm{~s}$ and an NO consumption rate of $3500 \mathrm{~s}^{-1}$.

Figure 5.1 shows simulation results of SMC [NO] levels utilizing a NO production rate of $1.0 \mathrm{pmol} / \mathrm{cm}^{2} \mathrm{~s}$ at a NO consumption rate of $3500 \mathrm{~s}^{-1}$. It is noted that at 
this combination of NO production and consumption rate, a peak NO concentration that is available in the SMCs is approximately $36 \mathrm{nM}$. Similar simulations were conducted with the varying NO production and consumption rate combinations, and the NO concentration levels within the SMCs were noted. Figure 5.2 represents the simulation data acquired for the different NO production rates while maintaining a constant consumption rate of $3500 \mathrm{~s}^{-1}$. It is noted that there is a linear relationship between NO production rate and NO concentration available in the SMCs. For the average NO production rate achieved at maximum agonist stimulation $\left(0.418 \pm 0.18 \mathrm{pmol} / \mathrm{cm}^{2} \mathrm{~s}\right)$, a corresponding SMCs NO concentration level obtained is $15.1 \mathrm{nM}$ (using a $\mathrm{k}_{\mathrm{bl}}$ of $3500 \mathrm{~s}^{-}$ $\left.{ }^{1}\right)$. Similar linear relationships were observed when evaluating the varying NO production rates with each constant $\mathrm{NO}$ consumption rate analyzed. 


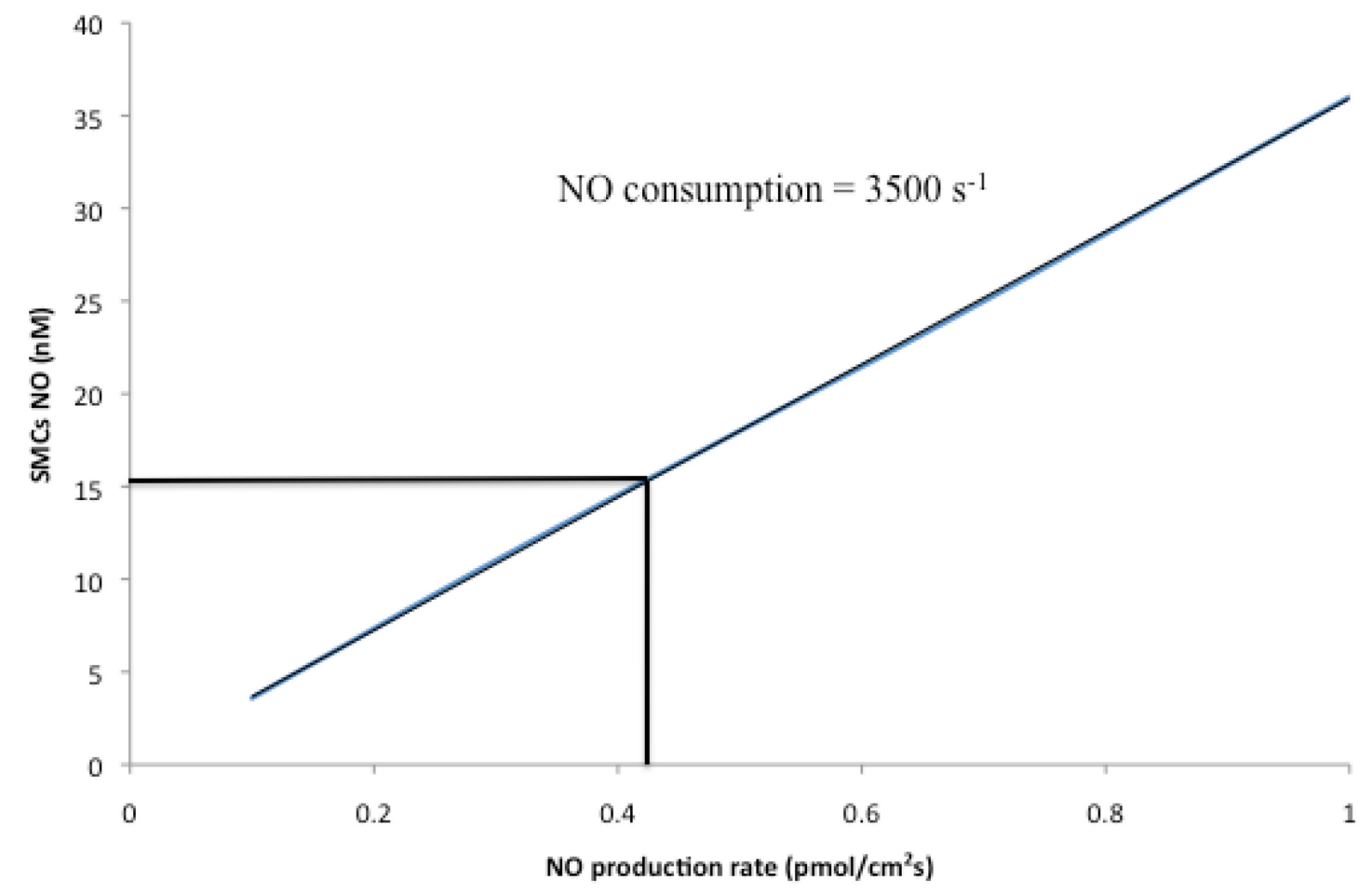

Figure 5.2 - Linear relationship between varying NO production rates and a SMCs NO concentration levels simulated by theoretical arteriole model assuming a constant NO consumption rate of $3500 \mathrm{~s}^{-1}$.

Figure 5.3 summarizes the simulation data obtained for all combinations of NO production and consumption rates. It is noted the NO production rate value has more of an effect on the available [NO] within the SMCs. As the NO production rate increases, there is a noticeable increase in SMCs [NO]. Interestingly, varying the NO consumption rates had very little effect on the overall SMC [NO]. Therefore it is concluded that the NO production rate plays a significant role in the amount of NO available in the SMCs. This can aid in explaining that when there is damage to the lining of the vasculature and more specifically to the ECs and site of NO production, the ability to regulate vascular tone is dramatically impaired. Further simulations were performed (not shown) to examine when the concentration of NO within the SMCs would be zero. This situation 
was noted to occur at NO production rates less than $0.001 \mathrm{pmol} / \mathrm{cm}^{2} \mathrm{~s}$. Therefore, this value was noted to be the threshold value. In order to observe physiological relevant [NO] levels within the SMCs, NO production rates had to be greater than this value.

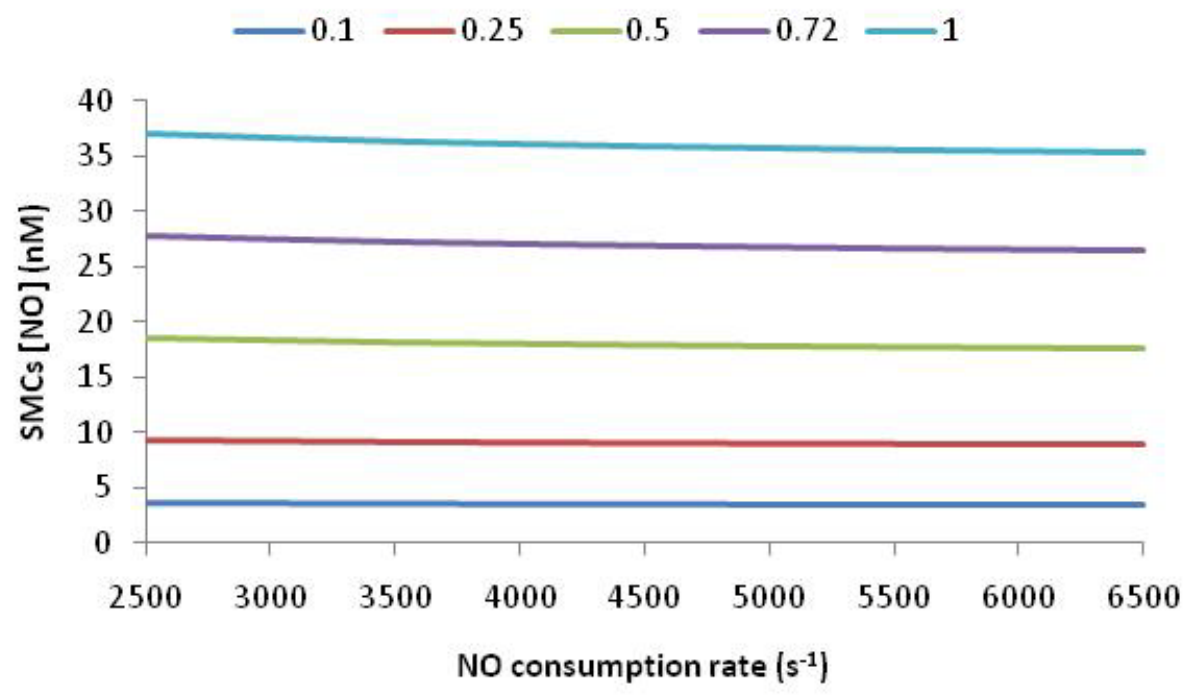

Figure 5.3 - Summarized data of simulations performed for varying combinations of NO production and consumption rates to evaluate SMC [NO]

Conclusion. In conclusion, the experimental and theoretical data achieved in this dissertation provided significant information in answering the "NO paradox" by accomplishing the aforementioned objectives. Combining the values of NO production rates and NO consumption rates determined, suggests that endothelial derived NO is in fact able to escape scavenging by RBCs where it then diffuses to the adjacent SMCs to induce vasodilation. The actual amount of bioavailable NO in the SMCs depends linearly on the endothelial NO production rate obtained. However, it has been previously suggested that the amount of NO required for the activation of the enzyme responsible for inducing vasodilation is within the range of $(1-250 \mathrm{nM})$ [4]. Our predicted SMC [NO] values are within this range. Further experimental work can be performed to examine 
production rates obtained upon stimulation at submaximal agonist levels. In addition, shear stress induced NO production could be evaluated. Also, NO consumption studies can be performed using human blood to see how reaction rates differ between porcine blood. However, in summary, this work provides proper methodologies on evaluating NO bioavailability in the SMCs by determining the balance between NO production and NO consumption in the vasculature, which can be utilized in further studies.

\subsection{References}

[1] S. Potdar and M. Kavdia, "NO/peroxynitrite dynamics of high glucose-exposed HUVECs: chemiluminescent measurement and computational model," Microvasc Res, vol. 78, pp. 191-8, Sep 2009.

[2] N. M. Tsoukias, et al., "A theoretical model of nitric oxide transport in arterioles: frequency- vs. amplitude-dependent control of cGMP formation," Am J Physiol Heart Circ Physiol, vol. 286, pp. H1043-56, Mar 2004.

[3] N. M. Tsoukias, et al., "Erythrocyte consumption of nitric oxide in presence and absence of plasma-based hemoglobin." Am J Physiol Heart Circ Phsiol, vol. 282, pp. H2265-H2277, 2002.

[4] C. X, et al., "The influence of radial RBC distribution, blood velocity profiles, and glycocalyx on coupled NO/O2 transport," J Appl Phsiol, vol. 100, pp. 482492, 2006. 


\section{SHABNAM M NAMIN}

\section{Education}

M.S. Industrial Engineering - Present

University of Miami

Miami, FL

M.B.A Business Administration - Present

University of Miami

Miami, FL

Ph.D. Candidate Biomedical Engineering - 2012

Florida International University

Miami, FL

B.S. Biomedical Engineering - 2006

Florida International University

Miami, FL

\section{Experience}

- President - S \& S Development of the TC, LLC - Opa-

July 2010 - Present Locka, FL

Develop and maintain several residential/commercial buildings in south Florida.

- President/Founder - BioBeauty Engineering - Miami, FL

August 2012 - Present

Development of tissue based products for wound healing applications

- Manufacturing Operations Specialist - BioTissue -

Feb 2010 - Nov 2010

Miami, FL

Worked in manufacturing and operations to produce a multi-targeted tissue based product used for healing applications. Performed validations and verifications on procedures and equipments. Assisted in SOP writing.

- Research Collaborator - Mount Sinai - Miami, FL

Collaborated on a research project studying the effects of shear stress on nitric oxide formation.

- Research Engineering - Bioheart - Sunrise, FL

June 2005 - Oct 2006

Worked in research and development, quality control and manufacturing, based on a novel cell-based therapy for patients who experienced myocardial infarctions. 


\section{Publications/Presentations}

- Namin, S., Nofallah, S., Joshi, M. S., Tsoukias, N., An experimental and theoretical study of DAF-FM activation by NO: Toward calibration of an NO-sensitive fluorescent dye. Nitric Oxide.

- Namin, S., Madrasi, K., Isaza, D., Tsoukias, N., Theoretical and experimental determination of nitric oxide consumption by red blood cells.

- Tercero, J. E., Namin, S., Lahiri, D., Balani, K., Tsoukias, N., Agarwal, A., Effect of carbon nanotube and aluminum oxide addition on plasma-sprayed hydroxyapatite coating's mechanical properties and biocompatibility. Mat. Sci. \& Eng, 2009. 29(7): p 2195-2202.

- Lahiri, D., Rouzaud, F., Namin, S., Keshri, A. K., Valdes, J.J., Kos, L., Tsoukias, N., Agarwal, A., Carbon nanotube reinforced polylactide-caprolactone copolymer: Mechanical strengthening and interaction with human osteoblasts in vitro. Mech. \& Mat. Eng, 2009. 1(11): p 2470-2476.

- BMES - An experimental and theoretical study of DAFFM activation by NO: Toward calibration of a NOsensitive fluorescent dye

- SBEC - Copolymer-born nitride nanotube composite for biodegradable scaffold application

- FASEB - An experimental and theoretical study of DAFFM action by NO: Toward calibration of an NO-sensitive fluorescent dye

- FASEB - Experimental and theoretical studies to determine smooth muscle nitric oxide availability

\section{Affiliations/Memberships}

- Tau Beta Pi

- Society of Women Engineers

- Biomedical Engineering Society
Manuscript under review

Manuscript under review

2009

2008

2006 - Present

2005 - Present

2004 - Present 